\title{
Adaptive Control of Wind Turbine Systems with Time-varying Parameters
}

\begin{tabular}{l} 
A Thesis \\
$\qquad$ Presented to \\
the Faculty of the School of Engineering and Applied Science \\
UNIVERITY OF VIRGINIA \\
\hline In Partial Fulfillment \\
of the Requirements for the Degree
\end{tabular}

CHI ZHANG

May 2015 
APPROVAL SHEET

The thesis

is submitted in partial fulfillment of the requirements

for the degree of

Master of Science

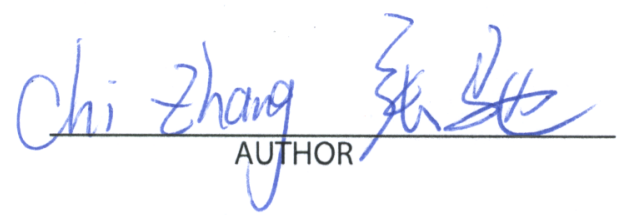

The thesis has been read and approved by the examining committee:

Prof. Gang Tao

\begin{tabular}{c}
\hline Advisor \\
Prof. Zongli Lin \\
\hline Prof. Scott Acton \\
\hline
\end{tabular}

Accepted for the School of Engineering and Applied Science:

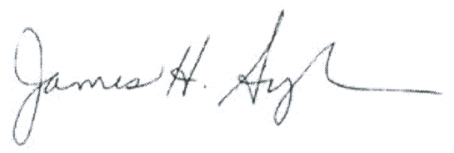

Dean, School of Engineering and Applied Science 


\section{Acknowledgements}

I would like to gratefully thank my advisor, Dr. Gang Tao, for his guidance, patience, persistent encouragement and continuous support during my graduate studies at the University of Virginia. He has challenged me continuously, meanwhile, providing me with the confidence that every task could be overcome. His deep knowledge of control theories and his attitude towards research have greatly inspired me to form a rigorous, dedicated, and creative research style, which is very helpful for my further work in the automatic field.

I am also very grateful that there are so many friendly colleagues and friends who have given me a lot of help on this thesis.

Finally, I want to thank my parents for their support and faith in me, without whom this thesis can’t be meaningful and possible. 


\section{Abstract}

The vibration problem on wind turbine blades is, at times, unavoidable. The unpredictable effects of blade vibrations on the modern high-capacity and large size wind turbine can harm the efficiency of wind power generation, as well as the safety operation on the wind farm. The objective of this research is to deal with the aeroelastic dynamics of the spinning blade with dynamic load, which means we need to deal with the time-varying parameters in the wind turbine system model. The system nonlinearities could limit the performance of the feedback control.

In this research, we develop an adaptive backstepping control design for singleinput and single-output wind turbine dynamic systems with the control input as the gurney flap which is deployed in the trailing-edge of the wind turbine blade. Our control scheme has the capacity to guarantee the desired system tracking performance in the presence of blade vibrations causing system parameters to be time-varying.

The model of an NACA0012 airfoil is presented for situations where two scenarios are studied: the nominal model with constant parameters and the actual model with time-varying parameters. The wind turbine dynamic system model used in our study is based on data taken from this airfoil model. We will first demonstrate with simulation results that an existing adaptive model reference control design can handle the scenario of the nominal model with constant parameters, but cannot achieve the desired system performance for the actual model with the time-varying system parameters. We will then show by both an analytic and simulation results that our developed adaptive backstepping control design effectively can handle the time-varying

parameters, ensuring tracking the desired system output (pitch angle), despite large system parameter variations. 


\section{Contents}

1 Introduction 1

1.1 Research Motivation ................... 1

1.2 Literature Review . . . . . . . . . . . . . . . . . . . . . . . 2

1.3 Thesis Outline . . . . . . . . . . . . . . . . . 4

1.4 Contributions in This Thesis . . . . . . . . . . . . . . 4

2 Background $\quad 6$

2.1 Modeling of Control Systems . . . . . . . . . . . . . 6

2.2 System Stability . . . . . . . . . . . . . . . . . . 7

2.3 Adaptive Control . . . . . . . . . . . . . . . . . . . . . . 10

3 Wind Turbine Systems 14

3.1 Basic Components of Wind Turbine Systems . . . . . . . . . . . . 15

3.1 Rotor .......................... 16

3.1 .2 Drive Train . . . . . . . . . . . . . . . . 17

3.1.3 Generator . . . . . . . . . . . . . . . . . 17

3.2 Modeling of Wind Turbine Systems . . . . . . . . . . . . . . . . . 18

3.2.1 Modeling of Wind Turbine Rotor Blade . . . . . . . . . . . . . 18

3.2.2 Modeling of Drive Train . . . . . . . . . . . . . . . 21

3.2.3 Modeling of Induction Generator . . . . . . . . . . . . . . 23 
3.3 Wind Turbine Systems with Gurney Flap . . . . . . . . . . . . . . . . 24

3.3.1 Gurney Flap Applications ................ 25

3.3.2 Hystereses in Smart Actuators . . . . . . . . . . . . 26

3.4 Dynamic Equation of Wind Turbine Systems . . . . . . . . . . . . . . 27

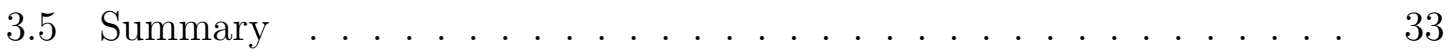

4 Study of Adaptive Model Reference Control Designs 34

4.1 State Tracking Control Design . . . . . . . . . . . . . . 34

4.1.1 State Tracking Controller Structure . . . . . . . . . . . . . 35

4.1.2 Limitations of State Tracking Control Design . . . . . . . . . 36

4.2 Output Tracking Control Design . . . . . . . . . . . . . . . . 37

4.2.1 Output Tracking Controller Structure . . . . . . . . . . . 38

4.2 .2 Adaptive Law Design . . . . . . . . . . . . . . . . . 39

4.2 .3 Stability Analysis . . . . . . . . . . . . . . . . 41

4.2.4 Simulation Study . . . . . . . . . . . . . . . . . 42

5 Adaptive Backstepping Control $\quad 48$

5.1 A Basic Adaptive Backstepping Scheme . . . . . . . . . . . . . . 49

5.1.1 Adaptive Backstepping Control Design . . . . . . . . . . . . . 49

5.1 .2 Simulation Study . . . . . . . . . . . . . . 53

5.2 Adaptive Backstepping Control for Wind Turbine System Model . . . 58

5.2.1 Adaptive Backstepping Control Design . . . . . . . . . . . . 60

5.2.2 Simulation Study . . . . . . . . . . . . . . . . . 64

5.3 Comparison Study . . . . . . . . . . . . . . . . 70

5.3.1 An Existing Adaptive Control Design . . . . . . . . . . . . 70

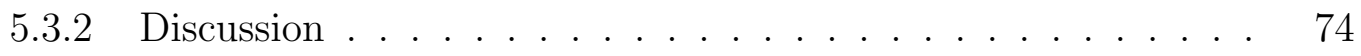

5.4 Summary . . . . . . . . . . . . . . . . . 75 
6 Conclusions and Future Work $\quad 76$

6.1 Summary and Conclusions . . . . . . . . . . . . . . 76

6.2 Future Work . . . . . . . . . . . . . . . . . 78

$\begin{array}{ll}\text { Bibliography } & 79\end{array}$ 


\section{List of Figures}

2.1 Adaptive control system. . . . . . . . . . . . . . . . . . . . . 11

2.2 Direct adaptive control system [4] . . . . . . . . . . . . . . . . . 12

2.3 Indirect adaptive control system $[4] \ldots \ldots \ldots \ldots \ldots$

3.1 Modern utility-scale wind turbine $[48] . \ldots \ldots \ldots \ldots \ldots$

3.2 Components of a horizontal axis wind turbine. . . . . . . . . 16

3.3 Cross section of wind turbine blade airfoil and angles [18]. . . . . 20

3.4 Rotational system with a gear train. . . . . . . . . . . . . . . 21

3.5 Doubly-fed induction generator in wind turbine from Mathworks. . . 23

3.6 Gurney flap in airfoil $[47] . \ldots \ldots \ldots \ldots \ldots \ldots$

4.1 Tracking error (Scenario I: nominal control, $\left.\omega_{r}=0\right) \ldots \ldots \ldots 42$

4.2 Tracking error (Scenario II: adaptive control, $\theta(0)=75 \% \theta^{*}, \Gamma=5$ ) 43

4.3 Parameter errors for $\theta_{1}, \theta_{2}, \theta_{3}$, when $\theta(0)=75 \% \theta^{*} \ldots \ldots \ldots$

4.4 Parameter errors for $\theta_{4}, \theta_{5}, \rho$, when $\theta(0)=75 \% \theta^{*} \ldots \ldots \ldots$

4.5 Scenario III: nominal control, $\omega_{r}=2 \ldots \ldots \ldots \ldots \ldots$

4.6 Tracking error (Scenario IV: adaptive control, $\left.\omega_{r}=2, \Gamma_{1}=0.5\right) \ldots 45$

4.7 Parameter errors for $\theta_{1}, \theta_{2}, \theta_{3}, \theta(0)=90 \% \theta^{*} \ldots \ldots \ldots$

4.8 Parameter errors for $\theta_{4}, \theta_{5}, \rho, \theta(0)=90 \% \theta^{*} \ldots \ldots \ldots$

4.9 Tracking error (Scenario V: adaptive control, $\left.\omega_{r}=2, \Gamma_{2}=5\right) \ldots 47$ 
4.10 Tracking error (Scenario VI: adaptive control, $\left.\omega_{r}=2, \Gamma_{3}=15\right) \ldots . . \quad 47$

5.1 Tracking error (nominal control, $\left.\omega_{r}=2, y_{m}=1, \theta(0)=\theta^{*}, \Gamma=0\right) \quad$. 53

5.2 Tracking error (adaptive design, $\left.\omega_{r}=2, y_{m}=1, \theta(0)=75 \% \theta^{*}, \Gamma=5\right) \quad 54$

5.3 Tracking error $\left(\omega_{r}=2, y_{m}=1, \theta(0)=85 \% \theta^{*}, \Gamma=5\right) \quad \ldots \ldots$

5.4 Tracking error $\left(\omega_{r}=2, y_{m}=\sin (t), \theta(0)=\theta^{*}, \Gamma=0\right) \quad \ldots \ldots$

5.5 Tracking error $\left(\omega_{r}=2, y_{m}=\sin (t), \theta(0)=75 \% \theta^{*}, \Gamma=15\right) \quad \ldots \quad . \quad 55$

5.6 Tracking error $\left(\omega_{r}=2, y_{m}=\sin (t), \theta(0)=85 \% \theta^{*}, \Gamma=12\right) \ldots \ldots$

$5.7 \quad$ Tracking error $\left(\omega_{r}=2, y_{m}=\sin (0.5 t), \theta(0)=\theta^{*}, \Gamma=0\right) \ldots \ldots 6$

5.8 Tracking error $\left(\omega_{r}=2, y_{m}=\sin (0.5 t), \theta(0)=75 \% \theta^{*}, \Gamma=10\right) \ldots \ldots$

5.9 Tracking error $\left(\omega_{r}=2, y_{m}=\sin (0.5 t), \theta(0)=85 \% \theta^{*}, \Gamma=10\right) \ldots \ldots$

5.10 Tracking error (nominal control, $\left.\omega_{r}=3, y_{m}=1\right) \ldots \ldots$

5.11 Tracking error (adaptive control, $\omega_{r}=3, y_{m}=1, \theta(0)=75 \% \theta^{*}$ ) . . $\quad 65$

5.12 Tracking error $\left(\omega_{r}=4, y_{m}=1, \theta(0)=85 \% \theta^{*}\right) \ldots \ldots 6$

5.13 Tracking error (nominal control, $\omega_{r}=3, y_{m}=\sin (t)$ ) . . . . . . 67

5.14 Tracking error (adaptive control, $\left.\omega_{r}=3, y_{m}=\sin (t), \theta(0)=75 \% \theta^{*}\right) .67$

5.15 Tracking error $\left(\omega_{r}=4, y_{m}=\sin (t), \theta(0)=85 \% \theta^{*}\right) \ldots \ldots 6$

5.16 Tracking error (nominal control, $\left.\omega_{r}=3, y_{m}=\sin (0.5 t)\right) \ldots . . . \quad 68$

5.17 Tracking error (adaptive control, $\left.\omega_{r}=3, y_{m}=\sin (0.5 t), \theta(0)=75 \% \theta^{*}\right) \quad 69$

5.18 Tracking error $\left(\omega_{r}=4, y_{m}=\sin (0.5 t), \theta(0)=85 \% \theta^{*}\right) \ldots \ldots$ 


\section{List of Tables}

3.1 System Parameters . . . . . . . . . . . . . . . . . . . . 28

5.1 System Zero Polynomials . . . . . . . . . . . . . . . . . . . . 60 


\section{Chapter 1}

\section{Introduction}

\subsection{Research Motivation}

\section{Wind turbine control problems}

Wind energy has been one of the fastest-growing energy sources in the world with its characteristics of inexhaustible feature and cleanness. Therefore, some recent research work has been done in the wind turbine system control designs to make turbine devices more efficient [2]. Nowadays, the wind turbines have become larger and more flexible. When the turbines need to be applied in more complicated environment, they will meet a myriad of control problems. No matter what types of wind turbines are, variable-speed or fixed-speed, they always have the complex inner control systems and have been studied by several research groups [12], [13].

\section{Wind turbine systems with time-varying parameters}

In [3], the authors develop a new version of control design on the turbine blades vibration problem. The vibration problem has been a big issue, which can harm the large-size wind turbine operation and decrease the efficiency of the wind power gener- 
ation. Furthermore, the vibration problem can cause unsteady loads with fluctuation of the flow over the rotating blade. Due to the problem of blade vibration, it is necessary to develop a new adaptive control design on this problem in order to achieve the vibration suppression purpose. In order to fix this problem, in this thesis, we will present a new approach of adaptive control design, which our controller can solve the vibration problem with time-varying wind turbine systems. Our adaptive backstepping control design is much more powerful to obtain the desired system performance, which means the wind turbine pitch angle can track the ideal reference signal.

\subsection{Literature Review}

Control problems in wind turbine systems. In [5], [6], [7], the nonlinear aeroslastic system has been presented. The aeroelastic two-dimensional wing section is investigated with trailing-edge and leading-edge parts. The governing equation of motion of the aeroelastic model was also be demonstrated in these papers. The results from these papers showed that their designed controller and adaptive laws were effective for the flutter suppression, and the considered damping uncertainty had positive effect on their control design.

Smart actuators in wind turbine systems. As the control input, one of the smart actuators, Gurney flap, has been illustrated in [31], [32], [33]. A slightly different in turbine dynamics is presented with this smart actuator. In order to take stock of recent research and development of Gurney flap, to be more effective, the Gurney flap should be mounted at the trailing edge perpendicular to the chord line of airfoil or wing. The flap height is related to the order of local boundary layer thickness. Similarly, in [34], based on the formal research study of Gurney flap, the deployment of another smart actuator, microtab, is also illustrated. For more different types of 
airfoils, an additional Gurney flap increases the pressure on the upstream surface of the airfoil, which increases the total pressure of the lower surface. Furthermore, due to the constrains of the materials in smart actuators, there were some hysteresis characteristics existing, which could cause the result of system oscillation or some undesirable inaccuracy. Therefore, in [28], [29], [41], [42], [43], authors developed several adaptive control designs to decrease the negative influences of hysteresis phenomenon in these smart actuators.

Specific problems in wind turbine systems. There has been little research study on solving the problem of the control of the blade rotation of wind turbine systems. Most research direction is mainly on the integral control of the whole wind turbine system, due to the difficulties of the modeling on the time-varying parameter in the wind turbine system. Basically, blade vibration is a big issue of wind turbine systems, in [3], the authors develop a method of output feedback control design for fixing this problem with several assumptions and the K-Y lemma in order to achieve the objective of vibration suppression. The simulation results show that the desired output, pitch angle, could track the chosen ideal reference signal.

Numerical simulations in wind turbine systems. Some other research focused on the numerical simulations in wind turbine systems in [18], [27], they provide the results that the efficiency of the wind turbine can be ideally achieved to nearly $60 \%$, which testify that the higher hypothesis on wind turbine power generation can't be realized in any control design situations. By presenting the results in numerical simulation way, we find that wind turbine systems would work normally under some conditions in [10], [15], [17]. There are three working regions which are referred as Region 1, Region 2 and Region 3, all the test simulations on the wind turbine airfoils should be finished in Region 2. In Region 1, there is no enough wind energy to 
supply the motivation to make the wind turbine systems start to work normally and in Region 3, the wind energy is so powerful that will destruct the wind turbine devices.

\subsection{Thesis Outline}

Our thesis is presented as follows. Chapter 2 provides the background needed to discuss about the adaptive output tracking and adaptive backstepping algorithms. Modeling of the control system, system stability and the adaptive control are going to be presented. In Chapter 3, we will discuss the wind turbine systems, in this chapter, basic components of wind turbine system, dynamics models and the gurney flap will be presented. Chapter 4 will present that the limitations of the state tracking, output tracking control design. Chapter 5 will present the basic adaptive backstepping control design, the application on wind turbine systems and the comparison with [3]. Chapter 6 will discuss the conclusions and some future work.

\subsection{Contributions in This Thesis}

In this section, we list the main contributions of this thesis.

1. We start a new research version in studying control problems of wind turbine systems, few papers have talked about control of the wind turbine systems with time-varying parameters. More advanced adaptive control design is introduced to make this new version more potential for wind turbine systems application.

2. We present a comprehensive study of adaptive control design for wind turbine systems, which is mainly focusing on solving the problem of time-varying system parameters. Compared with an existing adaptive control design, we study a 
new approach of applying adaptive backstepping control design to wind turbine systems.

3. Our adaptive backstepping control design can achieve the desired wind turbine system performance. The simulation results show that our new adaptive control design can obtain the desired asymptotic tracking, which means the system output, the pitch angle, can track a chosen reference signal having certain practical meaning.

4. As verified by extensive simulation results, our new adaptive backstepping control design can achieve the desired control objective, based on which, we can conclude that our study provides a desirable foundation for solving the problem of controlling wind turbine systems with time-varying parameters.

5. Besides, we generate some control problems in wind turbine systems with smart actuators. It is a new research area that we can improve the performance of those smart actuators, in order to make wind turbine systems more efficient, such as adaptive compensation of nonlinearities of smart actuators caused by hystereses of smart materials used in actuators. 


\section{Chapter 2}

\section{Background}

Before going further to discuss about wind turbine systems and our control designs, some background information should be introduced. In this chapter, modeling of control systems, control system stability, and the adaptive control are will be discussed.

\subsection{Modeling of Control Systems}

In [44], two approaches are presented to illustrate the analysis and design of feedback control systems. Here, we mainly discuss the state-space model method, which can be considered as the modern control modeling or time-domain approach. It is a specific way for modeling, analyzing, and designing more complex control systems, such as the nonlinear systems with backlash, saturation or dead-zone. The general state-space model is designed as

$$
\dot{x}=A x(t)+B u(t), y=C x(t)+D u(t),
$$


where $x(t) \in R^{n \times 1}$ is taken as the state vector, $A \in R^{n \times 1}$ is the system matrix, $B \in R^{n \times m}$ is considered as the input matrix. $u(t) \in R^{m \times 1}$ is the control input, respectively, $C \in R^{r \times n}$ is the output matrix, and $y(t) \in R^{r \times 1}$ is the system output. Plus, some control systems consider the $D \in R^{n \times m}$ as the feed-forward matrix. In our control design, $D$ matrix is always considered as zero.

For a time-varying system modeling, we still can consider a state-space equation,

$$
\begin{aligned}
& \dot{x}=A(t) x(t)+B(t) u(t), \\
& y=C(t) x(t)+D(t) u(t)
\end{aligned}
$$

where $A(t), B(t), C(t), D(t)$ have the same appropriate dimensions with some timevarying parameters. We will present the our adaptive control method by applying the modeling of the system in a state-space form to demonstrate the new control design procedure.

\section{$2.2 \quad$ System Stability}

In the control design strategy, it is crucial to make the system stable, an unstable control system is usually harmful and useless. Originally, to check the stability of a control system, the system transfer function is always introduced, which can be considered as the classical control of modeling [44]. But how to choose the control design method depends on the actual system model. It is general to demonstrate the modern control modeling strategy for most system models.

It is also possible to analyze the eigenvalues of matrix $A$ to see whether the system is stable, marginally stable or unstable. 
Lyapunov Stability. However, in the adaptive control design, these matrices parameters like $A, B, C$ are always unknown. So it is necessary to do the work with Lyapunov functions, which is also considered as the Lyapunov candidates, that are to deal with the problem of the system stability, which do not need to directly solve the results of the system. Generally, a Lyapunov candidate is chosen as some positive definite function, which can be $V(x, t)$. This Lyapunov function candidate is positive for any $x$ and $t$ and $V(0, t)=0$ for any $t \geq 0$, and evaluates the time derivative of $V(x, t)$. If $\dot{V}$, as a function of the time $t$, does not increase as $t$ increases, the equilibrium state of the system has the certain stability properties, where we can consider that the designed system is stable, $V(x, t)$ becomes a Lyapunov function.

Furthermore, some stability theories relevant to adaptive estimation and control designs are introduced: Signal norms, Gronwall-Bellman Lemma, Barbalat Lemma and Lefschetz-Kalman-Yakubovich Lemma.

Signal Norms. For a vector signal $x(t) \in R^{n}$, the $L^{1}, L^{2}$, and $L^{\infty}$ norms are defined as follows:

$$
\begin{aligned}
\|x(\cdot)\|_{1} & =\int_{0}^{\infty}\|x(t)\|_{1} d(t)=\int_{0}^{\infty}\left(\left|x_{1}(t)\right|+\cdots+\left|x_{n}(t)\right|\right) d t \\
\|x(\cdot)\|_{2} & =\sqrt{\int_{0}^{\infty}\|x(t)\|_{2}^{2} d t}=\sqrt{\int_{0}^{\infty}\left(x_{1}^{2}(t)+\cdots+x_{n}^{2}(t)\right) d t} \\
\|x(\cdot)\|_{\infty} & =\sup _{t \geq 0}\|x(t)\|_{\infty}=\sup _{\geq 0} \max _{1 \leq i \leq n}\left|x_{i}(t)\right| .
\end{aligned}
$$


The signal spaces $L^{1}, L^{2}$, and $L^{\infty}$ are defined as

$$
\begin{aligned}
L^{1} & =\left\{x(t) \in R^{n}:\|x(\cdot)\|_{1}<\infty\right\} \\
L^{2} & =\left\{x(t) \in R^{n}:\|x(\cdot)\|_{2}<\infty\right\} \\
L^{\infty} & =\left\{x(t) \in R^{n}:\|x(\cdot)\|_{\infty}<\infty\right\}
\end{aligned}
$$

And these are linear spaces which only contain elements with finite norms specified. The conclusion can be got if $x(t) \in L^{1} \cap \in L^{\infty} \Rightarrow x(t) \in L^{2}$.

Gronwall-Bellman Lemma. Let $f(t), g(t)$, and $k(t)$ be continuous and $g(t) \geq 0$, $k(t) \geq 0, \forall t \geq t_{0}$ and for some $t_{0} \geq 0$. If a continuous function $u(t)$ is given by

$$
u(t) \leq f(t)+g(t) \int_{t_{0}}^{t} k(\tau) u(\tau) d(\tau)
$$

Then $u(t)$ can be written in the form:

$$
u(t) \leq f(t)+g(t) \int_{t_{0}}^{t} k(\tau) f(\tau) e^{\int_{\tau}^{t} k(\sigma) g(\sigma) d(\sigma)} d(\tau)
$$

Gronwall-Bellman Lemma can bound the signals in a certain interval. It is difficult to find the signal bounded for a system, and harder to write a form like GronwallBellman Lemma. So, it is necessary to know the content of Barbalat Lemma, which will be more powerful for signal boundedness.

Barbalat Lemma. Barbalat Lemma is very useful for the signal boundedness and its derivatives. It is also very crucial for the system stability analysis. The following descriptions are known as Barbalat lemma.

Lemma 2.1 [Barbalat] If a scalar function $f(t)$ is uniformly continuous such that 
$\lim _{t \rightarrow \infty} \int_{0}^{t} f(\tau) d \tau$ exists and is finite, then $\lim _{t \rightarrow \infty} f(t)=0$.

Sometimes, it is not easy for us to check the uniform continuity of the signal, so the following lemma is more convenient for us to see the stability analysis, which is given by

Corollary 2.1 If $\dot{f}(t) \in L^{\infty}, f(t) \in L^{2}$, then $\lim _{t \rightarrow \infty} f(t)=0$.

Lefschetz-Kalman-Yakubovich Lemma. The following lemma is for the LefschetzKalman-Yakubovich Lemma. The matrix $A$ such that $\operatorname{det}(s I-A)$ has only zeros in the open left half plane, with $\delta>0$, a real $b$ such that $(A, b)$ is completely controllable, a real vector $c$, a scalar $d$, and a real symmetric positive definite matrix $L$. Then a real vector $q$ and a real matrix $P=P^{T}>0$, which satisfies that

$$
A^{T} P+P A=-q q^{T}-\delta L, P b-c^{T}=\sqrt{2 d} q
$$

when $\delta$ is small enough.

\subsection{Adaptive Control}

Adaptive control, as the name implies, applies physical adaptation dynamics that matches a controller for a system with the unknown system parameters to reach the goal which is the desired system performance. Differing from the classical control method, an adaptive control system can achieve more possible system operation that is much more flexible than the classical control. 


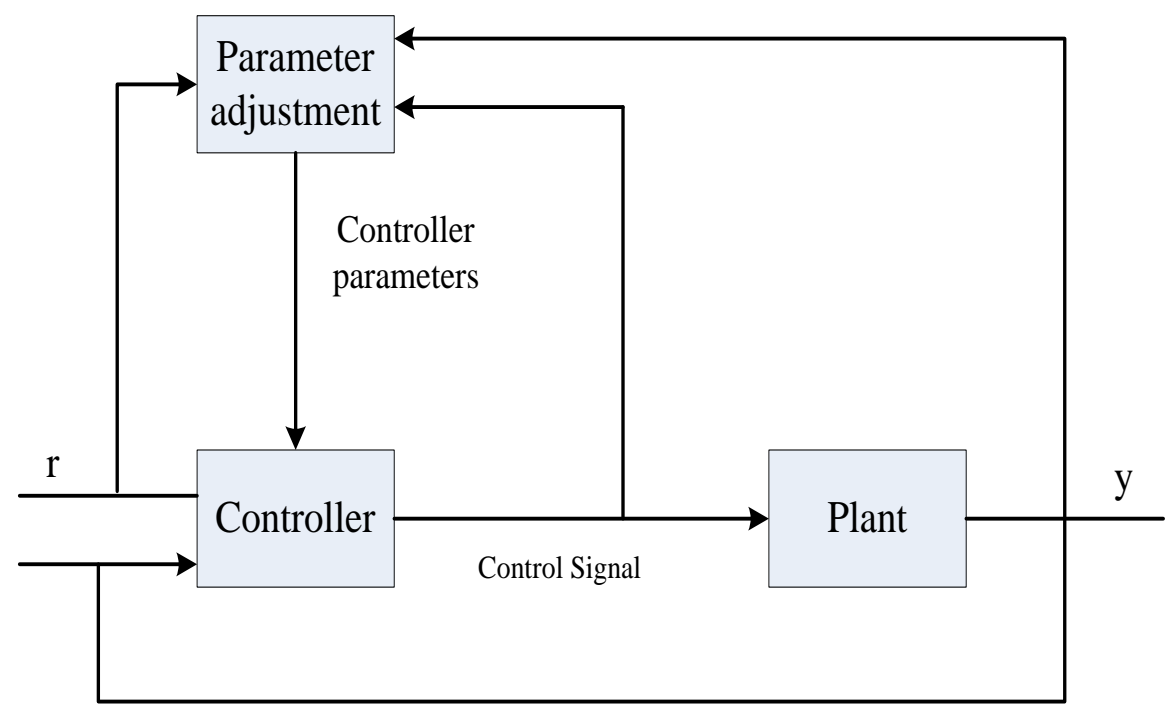

Figure 2.1: Adaptive control system.

\section{Direct adaptive control}

There are two ways to determine the adaptive control design. One is considered as the direct adaptive control and is to generate the updated system parameters with adaptive controller and adaptive laws without thinking the initial conditions of the target plant and possible disturbances. In a direct adaptive control system, the controller parameters, which are estimates of some unknown ideal plant-model controller matching parameters, are directly updated from adaptive laws, based on our defined tracking error. 


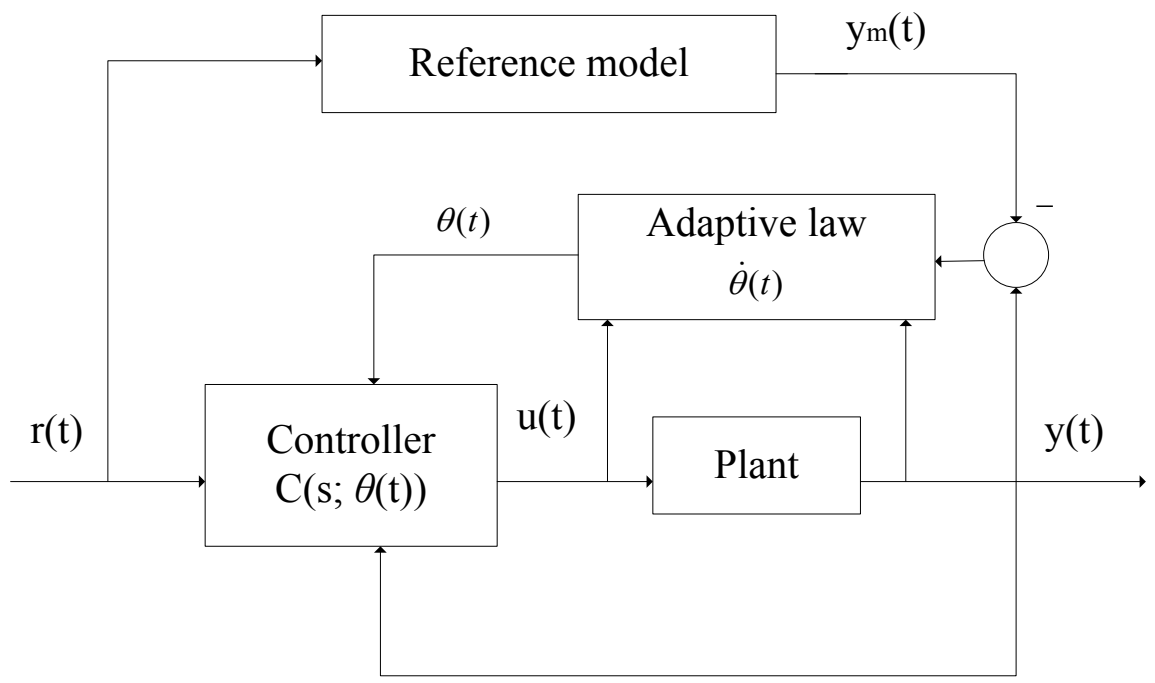

Figure 2.2: Direct adaptive control system [4].

\section{Indirect adaptive control}

The other one is called indirect adaptive control, this method is to estimate plant parameters with the possible disturbances, and update the adaptive controller with the designed adaptive laws. In an indirect adaptive control system, the controller parameters, which are also estimates of some unknown ideal plant-model matching parameters, are simultaneously calculated from a design equation using the on-line estimates of the unknown plant parameters, updated from a parameter estimator based on an estimation error presenting the mismatch between the plant output and its estimated version generated from the parameter estimation. 


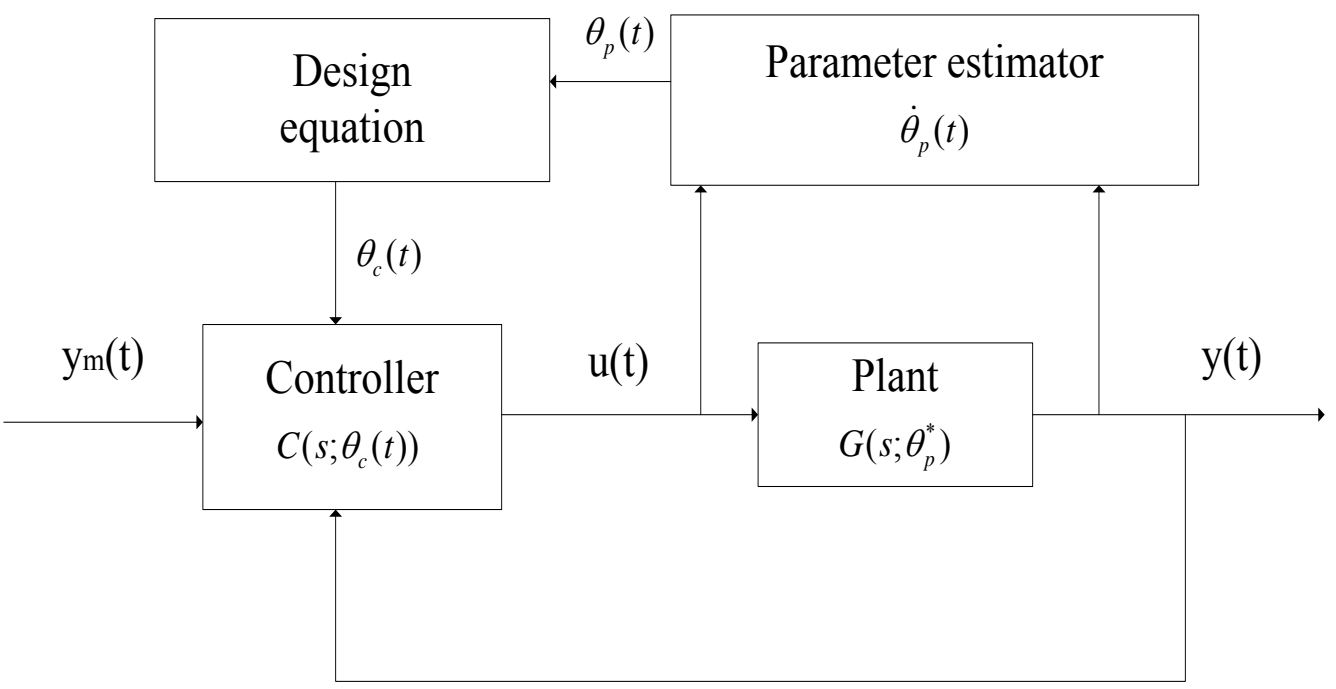

Figure 2.3: Indirect adaptive control system [4].

In either a direct or an indirect adaptive control design, it is important to use the estimates of some unknown parameters of an ideal controller as if they are the true values of those unknown parameters. The parameter estimates are obtained from an adaptive updated law driven by system performance error, and used in implementing an adaptive controller either directly or through a design equation to map the plant parameter estimates to the controller parameters. 


\section{Chapter 3}

\section{Wind Turbine Systems}

In this chapter, we will introduce the wind turbine systems. Many research groups are being studying the related topics about the wind turbine systems. In [12], the wind turbine is described as a machine that can convert the power from the wind to electricity. Some energy institutions have shown their statistics that the United States may receive $20 \%$ of its electrical power from wind energy by 2030 [13]. The number of this target is about $300 G W$ installed capacity. Since the energy crisis of the 1970s, a large-scale wind power plant has become a popular topic to all over the world [14]. A typical modern wind turbine of a large-scale wind power plant, in a wind farm configuration, which is related to a utility network, is shown in Figure 3.1.

Today, the most general design of the wind turbine is the horizontal axis wind turbine (HAWT) [15]. To understand well how the wind turbines are used, it is necessary to know some fundamental parts which compose the wind turbine systems. The mathematical modeling of the wind turbine systems provides a physical process to illustrate that the airfoil lift first will produce a pure positive torque on a rotating shaft, then the production of mechanical power will be transferred to electricity by a generator. In this chapter, we will also talk about smart actuators in wind turbine 


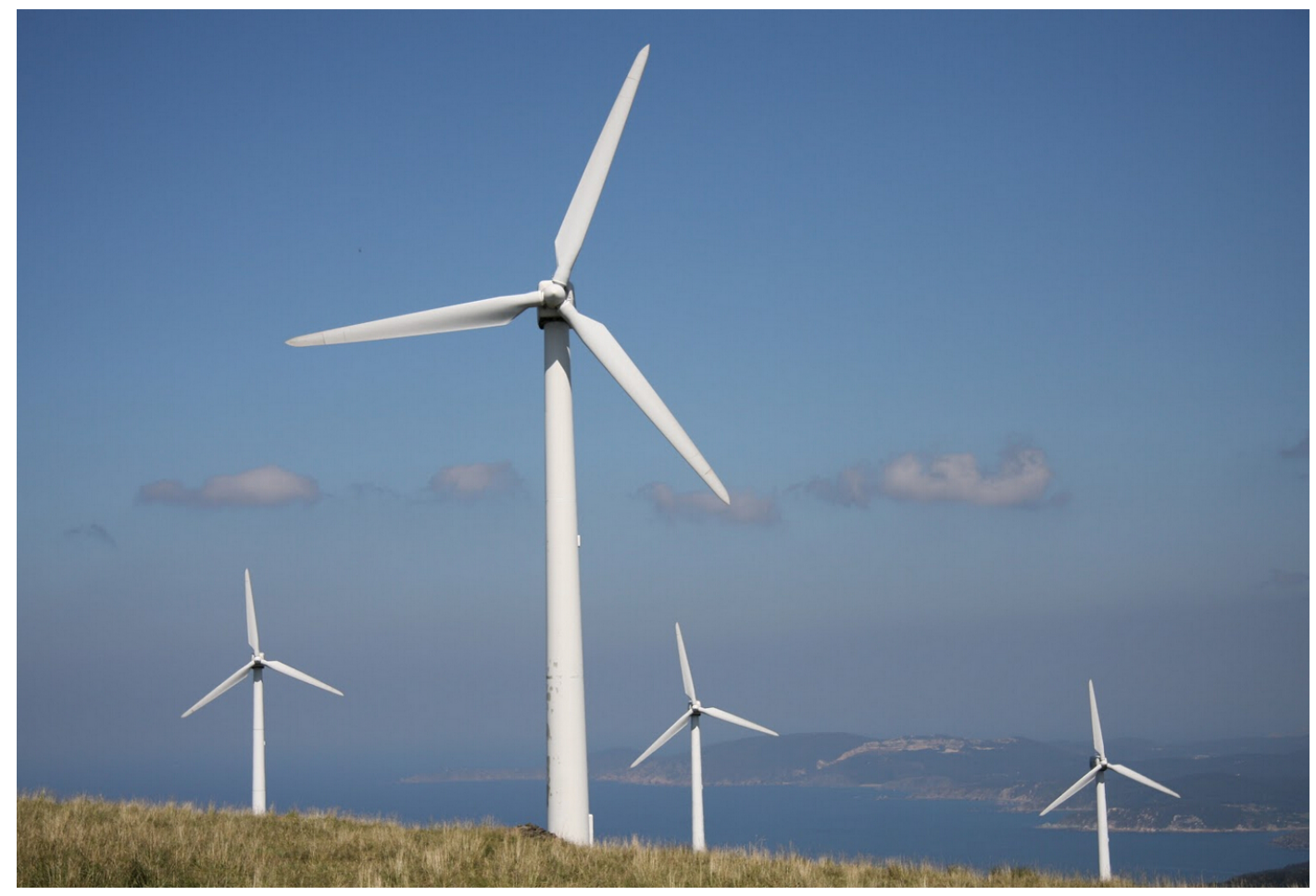

Figure 3.1: Modern utility-scale wind turbine [48].

systems. With the development of the wind turbine field, there are a variety of methods on actuators of wind turbine blade, which mainly focus on how to improve the efficiency of the wind turbine systems. At the end of this chapter, we will present the dynamic modeling of the wind turbine systems, which will be utilized in our adaptive control designs.

\subsection{Basic Components of Wind Turbine Systems}

As shown in Figure 3.2, the principal subsystems of HAWT are presented. And they include following elements [12]: 


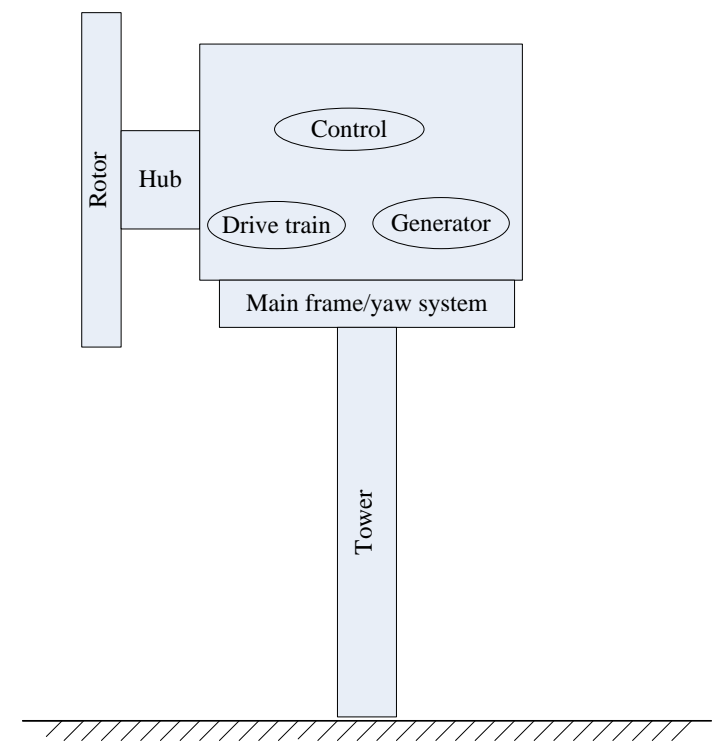

Figure 3.2: Components of a horizontal axis wind turbine.

- blades: horizontal, vertical and savonius;

- rotor: downwind and upwind types;

- hub: rigid, teetering, or hinged;

- power control by aerodynamic control (stall control) or variable-pitch blades (pitch control);

- synchronous or asynchronous generator (squirrel cage or doubly fed);

- drive train.

A compact summary will be done to overview some main components.

\subsubsection{Rotor}

There are two parts for the wind turbine rotor, which are the hub and blades. Nowadays, most turbines have upwind rotors with three blades and several downwind 
rotors are using under some certain conditions. The material of turbine blades are always composites, fiberglass or carbon fiber reinforced plastics.

\subsubsection{Drive Train}

The other rotating parts of the wind turbine downstream of the rotor make up of the drive train. They include a low-speed shaft(on the rotor side), a gearbox, and a high-speed shaft(on the generator side). The support bearings, one or more couplings, a brake, and the rotating parts of the generator are the other components of the drive train. The function of the gearbox is to increase the speed rate of the rotor rotation from a low value(tens of rpm) to a rate suitable for driving a generator(thousands of rpm). Parallel shaft and planetary are normally used in the wind turbine design. Fluctuating winds and the dynamics of large rotating rotors impose varying loads on drive train.

\subsubsection{Generator}

Most wind turbines use the induction or synchronous generators, when the power electronic converters are used with the generator, this kind of turbine is the type of the so-called variable-speed wind turbine [17].

Squirrel cage induction generators are installed in the most wind turbines. A squirrel cage induction generator operates within a narrow range of speeds slightly higher than its synchronous speed. This type of induction generator is rugged, cheap and easy to operate with an electrical network. The other type is called doubly fed induction generator. It is always used in the variable-speed wind turbine design.

An increasingly popular option for utility-scale electrical power generation is the variable-speed wind turbine. There are a number of profits that such a configuration offers, including the reduction of wear and tear on the wind turbine and potential op- 
eration of the wind turbines at maximum efficiency over a wide range of wind speeds, yielding increased energy capture. When using with suitable power electronic converters, either synchronous or induction generators of either type can run at variable speed.

\subsection{Modeling of Wind Turbine Systems}

As we know, a wind turbine device can transfer kinetic energy from the wind to mechanical energy. The key point in this process depends on the extraction between the rotor and the wind. The mean power output is determined by the mean wind speed, thus only steady-state aerodynamics have been considered to be important in this project and turbulence has been ignored. So it is important to understand some major aspects of wind turbine performance, such as power output and loads, which are influenced by the forces generated by the wind [19].

It is important to notice that we must focus on integrating wind power into the power systems. In above the section, we know that there are several important components in the wind turbine systems. So we present the rotor blade modeling, drive train modeling and induction modeling in this part.

\subsubsection{Modeling of Wind Turbine Rotor Blade}

In $[20,21]$, the original aerodynamic analysis of wind turbines was carried by Betz and Glauert from 1920s to 1930s. The wind power production equation is like

$$
P_{\text {wind }}=\frac{1}{2} \rho A V_{\text {wind }}^{3}
$$

where $\rho$ is air density, $A$ is the area swept by blades, $V_{\text {wind }}$ is the wind speed. 
From [20], it is known the maximum power which can be extract by an ideal wind turbine rotor with certain number blades from wind under ideal conditions is $59.26 \%$ of the power generated by wind. Generally, in practice, this number is closer to $50 \%$, limited by the number of blades and some constraints in economic cost or rapid changes of external environment. We definite the ratio of the extractable power to available power is the rotor power coefficient $C_{p}$. (3.1) can be rewritten as

$$
P_{\text {wind }}=\frac{1}{2} C_{p} \rho A V_{w i n d}^{3}
$$

Generally, equation (3.2) is given by as follows [18]

$$
P_{\text {rotor }}=\frac{1}{2} C_{p}(\lambda, \beta) \rho A V_{\text {wind }}^{3}
$$

where $\lambda$ is called tip-speed ratio(TSR) and $\beta$ is the blade pitch angle, the blade pitch angle is defined as the angle between the plane of rotation and the blade cross-section chord. The TSR is defined as

$$
\lambda=\frac{\omega_{\text {rotor }} \times R_{\text {rotor }}}{V_{\text {wind }}}
$$

where $\omega_{\text {rotor }}$ is the rotor angular speed $(\mathrm{rad} / \mathrm{s}), R_{\text {rotor }}$ is the rotor radius of the blade length.

So the rotor torque can be derived as

$$
T_{\text {rotor }}=\frac{\frac{1}{2} \rho C_{p} A V_{\text {wind }}^{3}}{\omega_{\text {rotor }}}
$$

where $A=\pi R_{\text {rotor }}^{2}$.

Normally, modern utility-scale wind turbines use airfoils, such as NACA0012, and 
so on, to utilize the kinetic energy from the wind. There are two forces acting on the airfoil:lift and drag. Wind turbines depend on lift force to apply torque to rotor blades, and also some torque caused by the drag force. Figure (3.3) shows lift and drag force on the airfoil model and some relevant angles which will be applied in the following sections. In Chapter 2, we consider a state-space model like

$$
\begin{aligned}
& \dot{x}=A(t) x(t)+B(t) u(t), \\
& y=C(t) x(t)
\end{aligned}
$$

where the system output $y$ can be found from Figure (3.3), which is the blade pitch angle.

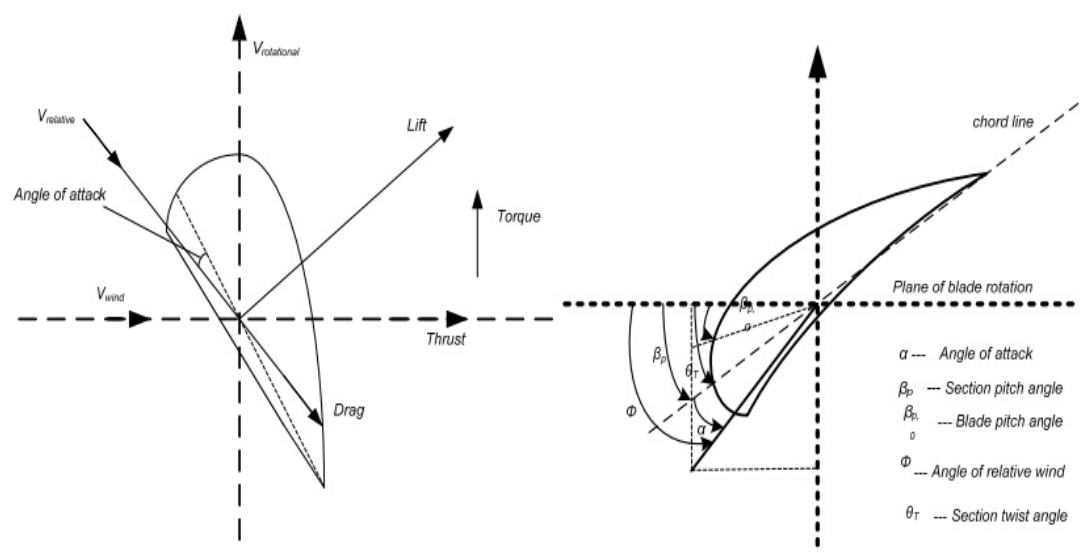

Figure 3.3: Cross section of wind turbine blade airfoil and angles [18].

The lift force is vertical to the effective airflow direction, which is directly applied on the torque that rotates for the rotor. The drag is perpendicular to the lift force on the cross section of the airfoil. 


\subsubsection{Modeling of Drive Train}

The drive train is the part of mechanical process of the wind turbine system. It consists of a rotor shaft, a blade pitching mechanism, a hub with blades, generator shaft and a gearbox. The shafts and the gearbox are using a two-mass inertia. In a rotational system [22], it is made up with a disk with a moment of inertia $J$ on a shaft. We assume that the viscous friction coefficient, as damping coefficient, is $D$ and the shaft torsional spring constant, which is stiffness, is $K$. So the torque acting on the disk can be obtained by the following equation, which is like

$$
T_{D}=J \frac{d^{2} \theta(t)}{d t^{2}}+D \frac{d \theta(t)}{d t}+K \theta(t)
$$

Next, we present two systems are connected through a gear train, and $T_{D}$ is the external torque on the disk of system 1, which is shown in Figure 3.4a.

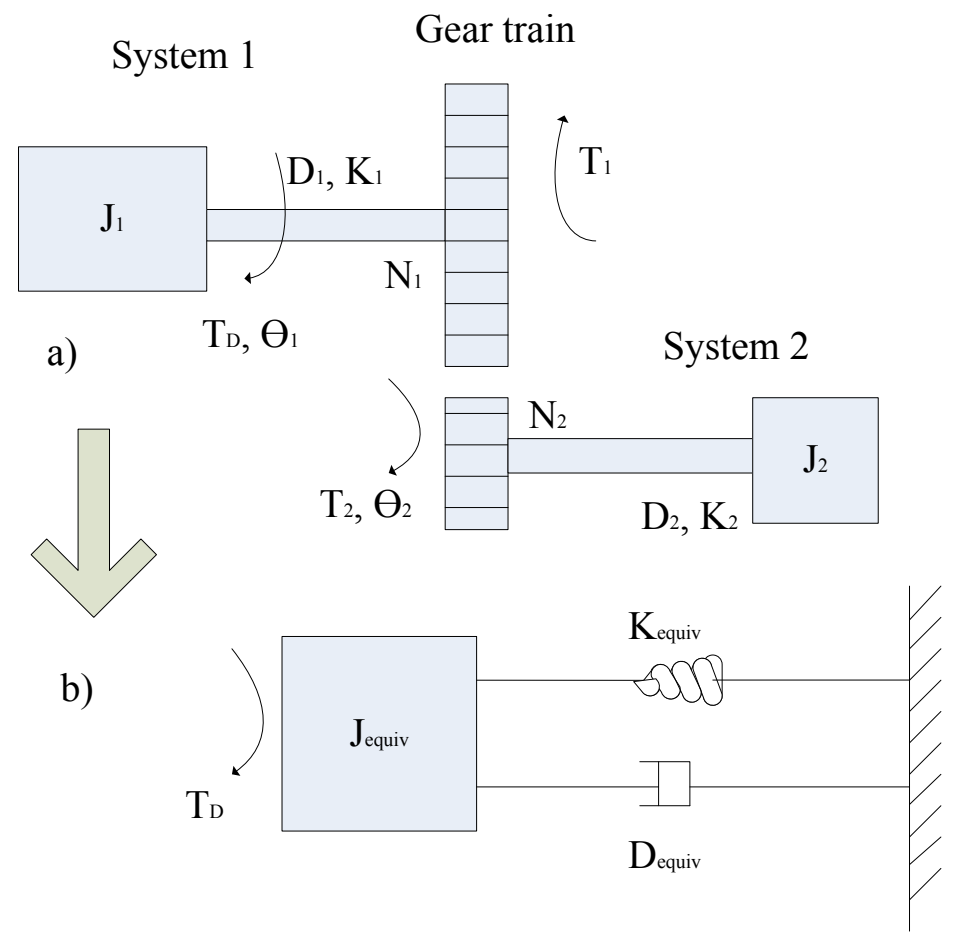

Figure 3.4: Rotational system with a gear train. 
The defined torque $T_{1}, T_{2}$ are transmitted torques. $N_{1}$ and $N_{2}$ are the number of teeth of gear 1 and gear 2 .

Using equation (3.7), the torque equation at $J_{1}$ is

$$
T_{D}=J_{1} \frac{d^{2} \theta_{1}}{d t^{2}}+D_{1} \frac{d \theta_{1}}{d t}+K_{1} \theta_{1}
$$

Similarly, the torque at $J_{2}$ is

$$
T_{2}=J_{2} \frac{d^{2} \theta_{1}}{d t^{2}}+D_{2} \frac{d \theta_{1}}{d t}+K_{2} \theta_{2}
$$

where $T_{1}=\frac{N_{1}}{N_{2}} T_{2}, \theta_{2}=\frac{N_{1}}{N_{2}} \theta_{1}$, we can connect the quantity relationship between gear 1 and gear $2[22]$, which $T_{1}$ can be written as

$$
\begin{aligned}
& T_{1}=\left(\frac{N_{1}}{N_{2}}\right)\left(J_{2} \frac{d^{2} \theta_{1}}{d t^{2}}+D_{2} \frac{d \theta_{1}}{d t}+K_{2} \theta_{2}\right) \\
& T_{1}=\left(\frac{N_{1}}{N_{2}}\right)\left[J_{2}\left(\frac{N_{1}}{N_{2}}\right) \frac{d^{2} \theta_{1}}{d t^{2}}+D_{2}\left(\frac{N_{1}}{N_{2}}\right) \frac{d \theta_{1}}{d t}+K_{2}\left(\frac{N_{1}}{N_{2}}\right) \theta_{1}\right]
\end{aligned}
$$

Then with (3.8)-(3.10), we can generate the equivalent system in Figure 3.4b as

$$
T_{D}=J_{\text {equiv }} \frac{d^{2} \theta_{1}}{d t^{2}}+D_{\text {equiv }} \frac{d \theta_{1}}{d t}+K_{\text {equiv }} \theta_{1}
$$

where

$$
\begin{gathered}
J_{\text {equiv }}=J_{1}+J_{2}\left(\frac{N_{1}}{N_{2}}\right)^{2} \\
D_{\text {equiv }}=D_{1}+D_{2}\left(\frac{N_{1}}{N_{2}}\right)^{2} \\
K_{\text {equiv }}=K_{1}+K_{2}\left(\frac{N_{1}}{N_{2}}\right)^{2}
\end{gathered}
$$




\subsubsection{Modeling of Induction Generator}

As we mentioned, there are two types of the wind turbine device: fixed-speed and variable-speed. For the fixed-speed type, most wind turbines will apply the squirrelcage induction generators. In [23], users may develop third or fifth-order algebraic models for induction machines. And for the variable-speed type, The doubly-fed induction generators are always used. The doubly-fed induction generator model can be expressed in the stationary stator reference frame, the reference frame rotating at rotor speed and the synchronously rotating reference frame. In [24, 25], the authors explained the synchronously rotating reference frame in order to simplify the controller design according to the fact that all the currents and voltages expressed under this reference frame will be of a DC nature. While, in [26], both stator and rotor variables were referred to their corresponding natural reference frames, and the machine model expressed in such reference frame is called the Quadrature-Phase Slip-Ring model. Figure 3.5 shows the whole working process of doubly-fed induction generator(DFIG).

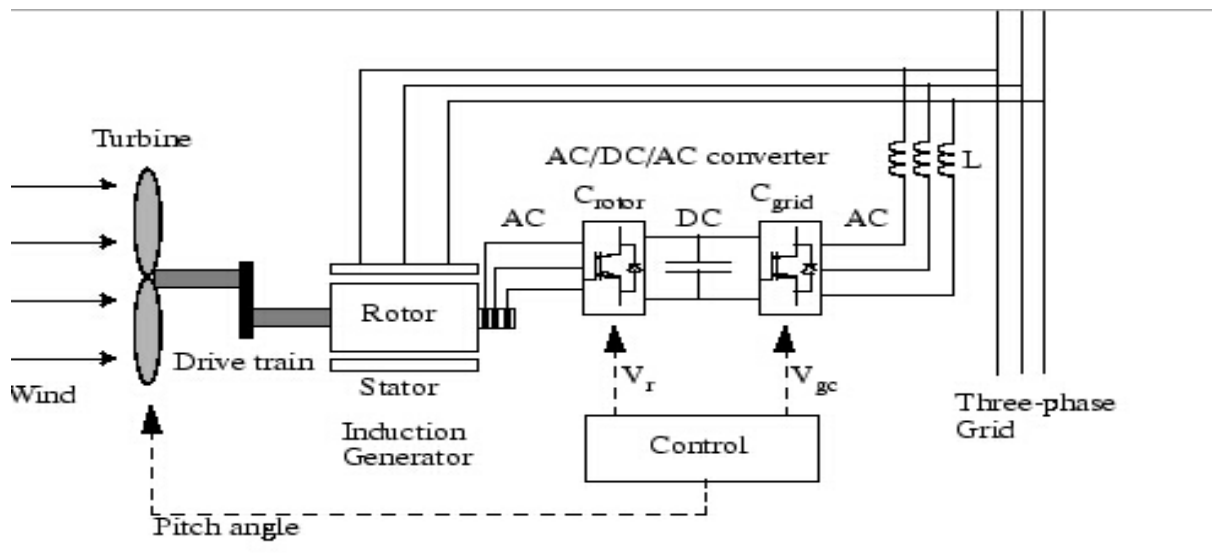

Figure 3.5: Doubly-fed induction generator in wind turbine from Mathworks. 


\subsection{Wind Turbine Systems with Gurney Flap}

Typically, the wind turbine blade model is an aeroelastic model, which has twodimensional wing section with both trailing-edge(TE) and leading-edge(LE). This model is discussed in [5], furthermore, in [3], the authors develop a new model with the actuator, gurney flap, which can provide the control force. It is always being put on the position of TE on the wind turbine airfoil. The effect of this nonlinear aeroelastic system is difficult to analyze since the fluid motion is generated by equations where analytical solutions are non-existent $[6,7]$. A full-nonlinear aerodynamic code solving the Euler equations has been coupled to a structural model for a two-dimensional flow case by Djayapertapa et al. [8,9].

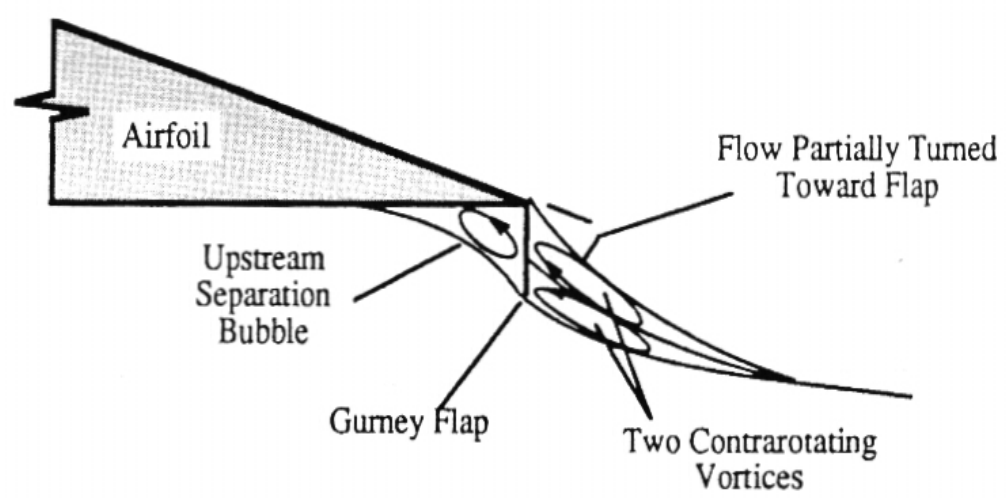

Figure 3.6: Gurney flap in airfoil [47].

From both $[5,6]$, we generate the data on the effects of the vibration with the parameters of the wind turbine systems in different blade rotation situations. It is generally from the parameter of the system based on the airfoil NACA0012, which is obtained by Thomas W. Strganac, Jeonghwan Ko, David E. Thompson [1] and some numerical study is done in [27].

As mentioned at the beginning of this chapter, [3] illustrates that an actuator 
called gurney flap provides the control input, so next, the application of gurney flap will be presented which includes the enhancement and mechanism in wind turbine systems. Also, as one of the smart material in wind turbine control, the phenomenon of hysteresis, which is in $[28,29]$, will also be introduced.

\subsubsection{Gurney Flap Applications}

Over the years, high-lift devices have attracted the attention of wind turbine designers and engineers as they can improve the efficiency of power production economically [30,31]. Flow over flaps can be very complicated, it involves boundary layers, main wind wakes, potential flow outside the boundary layer and flow in the flap shot. Here, we just illustrate some general idea about the Gurney flap characteristics.

The gurney flap is usually applied on the active flow control(AFC) process and this device involves small tabs located near the TE of an airfoil. The tabs are always put nearly perpendicular to the airfoil surface to a height on the order of the boundary layer thickness (1-2 \% chord). This trivial move can cause an effect on the aerodynamics of the airfoil by changing the Kutta condition [32], which is the point of flow separation, altering the TE flow conditions and changing the shape of the flow near the airfoil. The possible lift enhancement is achieved by deploying the tab on the lower surface and lift mitigation is obtained by deploying the tab on the upper surface.

The concept of the deployment of the gurney flap was conducted by researchers at UC Davis, they did both computational and experimental studies on lower surface, the effects of flap height, flap location and flap spacing were investigated for 3-D applications. The simulation results show that the flap of $1 \%$ of chord in height, located at $\mathrm{x} / \mathrm{c}=95 \%$ on the lower surface can achieve the best performance for lift enhancement, drag mitigation, and volume constraints [33,34]. In [35], the authors 
developed more comprehensive 2-D computational experiments for testing flap height and location on both upper and lower surface on the different wind-tunnel experimental airfoils, which are S809 and GU25-5(11)8. The optimal location for the lower surface was also $95 \%$ of chord with a height on the order of the boundary layer thickness, nearly $1 \%$ of chord. These different computational studies were testified on the airfoil $S 809$.

\subsubsection{Hystereses in Smart Actuators}

As one of the most effective smart actuators in wind turbine applications, gurney flap has been studied by different methods. In last section, researchers did a lot of work on the deployment position of the gurney flap on the testing airfoils, such as NACA0012, S809 and so on. These studies prove that the gurney flap can avoid the flow separation and move stall occurrence to higher angles of attack. However, the gurney flap itself has the characteristics of the piezoelectric and electromagnetic materials [40]. In this chapter, we will present the hysteresis characteristics and a brief introduction of the adaptive control design for hysteresis phenomena.

The hysteresis phenomenon appears in smart materials, such as piezoelectrics, shape memory alloys, and magnetostrictives. When these phenomena become strong in these materials hinders, there will be a serious influence on the effective use in actuators [36] and they often limit system performance, which can cause the inaccuracy or oscillations of the working process. The hystereses can be caused by stiction, magnetism or gears with backlash exist in physical systems [37]- [39]. The hysteresis characteristics are non-differentiable nonlinearities and unknown.

It is a hard task to develop an adaptive control scheme for systems with these unknown nonlinearities. In [41], the controlled plant is made up with a linear part precede by a hysteresis characteristic, which means, the hysteresis part is at the ac- 
tuator of a linear part. This linear part can be known or unknown, and the hysteresis is an unknown part. The authors would like to achieve the objective that with this unknown hysteresis part, the system can be stabilized and tracked. The control design is called the "adaptive inverse" method [42,43]. In $[42,43]$, the adaptive control of plants with non-differentiable nonlinearities, such as backlash, was applied. The authors presented an adaptive hysteresis inverse cascaded with the plant to cancel the effects of hysteresis so that the remaining part of the controller can keep its linear structure. The simplified model is also presented in [41], which contains most hysteresis characteristics. It is very effective to cancel the effects of hysteresis phenomena by using the adaptive hysteresis inverse control.

\subsection{Dynamic Equation of Wind Turbine Systems}

The dynamic blade model to be discussed is an aeroelastic model, which is given by $[5,6]$. It is also a pitch and plunge system coupled with unsteady aerodynamic loads. In our study, the actuator is gurney flap, which provides the control force and the system dynamic motion is down-forward plunging, which is referred as plunge displacement $h$ and nose-down pitching, defined as pitch angle $\theta$.

\section{Basic Equation for Aeroelastic Model}

The basic equation of the model is given by

$$
\left(\begin{array}{cc}
m & m x_{\theta} b \\
m x_{\theta} b & m
\end{array}\right)\left(\begin{array}{c}
\ddot{h} \\
\ddot{\theta}
\end{array}\right)+\left(\begin{array}{cc}
K_{h} & 0 \\
0 & K_{h}
\end{array}\right)\left(\begin{array}{c}
h \\
\theta
\end{array}\right)=\left(\begin{array}{c}
-L \\
M
\end{array}\right)
$$

where $m$ is the mass of blade section, $I_{\theta}$ is pitch moment of inertial about elastic axis, $b$ is semi-chord, $x_{\theta}$ is distance from elastic axis to mass center, $C_{h}, C_{\theta}$, are plunge and 
pitch damping coefficients, and $K_{h}, K_{\theta}$ are plunge and pitch spring stiffness. The lift aerodynamics $L$, and the moment $M$ with trailing-edge gurney flap are defined as

$$
\begin{aligned}
L & =\rho U^{2} b C_{1, \theta}\left(\theta+\frac{\dot{h}}{U}+\left(\frac{1}{2}-a\right) b \frac{\dot{\theta}}{U}\right)+\rho U^{2} b C_{1, g} g \\
M & =\rho U^{2} b^{2} C_{g, \theta}\left(\theta+\frac{\dot{h}}{U}+\left(\frac{1}{2}-a\right) b \frac{\dot{\theta}}{U}\right)+\rho U^{2} b^{2} C_{g, g} g
\end{aligned}
$$

where $\rho$ is air density, $U$ is wind velocity, $a$ is distance from mid-chord to elastic axis, $g$ is the gurney flap which provides the control force, and $C_{1, \theta}, C_{g, \theta}, C_{1, g}, C_{g, g}$ are lift and moment coefficients, angle of attack and gurney flap.

Most data of the system model based on the airfoil NACA0012, which has been mentioned in Chapter 3, can be found in Table I [5]. The system parameters $C_{1, \theta}$ and $C_{g, \theta}$ are corresponding to the periodical change of angle of attack when the blade is spinning. So the these two system parameters are supposed to be known as the sinusoid part with the same frequency of the blade rotation [3]: $C_{1, \theta}=3.375+$ $3.375 \sin \left(\omega_{r} t\right)$ and $C_{g, \theta}=(0.5+a) C_{1, \theta}$. The units are per radian.

Table 3.1: System Parameters

\begin{tabular}{|l|l|l|}
\hline \hline Symbol & Description & Value \\
\hline $\mathrm{a}$ & distance from airfoil mid-chord to elastic axis & $-0.6847 \mathrm{~m}$ \\
$\mathrm{~b}$ & airfoil semi-chord & $0.135 \mathrm{~m}$ \\
$\mathrm{~m}$ & mass of the airfoil model & $12.387 \mathrm{~kg}$ \\
$\rho$ & air density & $1.225 \mathrm{~kg} / \mathrm{m}^{3}$ \\
$I_{\theta}$ & mass moment of inertia of the wing about elastic axis & $0.065 \mathrm{~m}^{2} \mathrm{~kg}$ \\
$x_{\theta} b$ & distance between elastic axis and mass center & $0.033 \mathrm{~m}$ \\
$C_{h}$ & plunge structural damping coefficient & $27.43 \mathrm{~kg} / \mathrm{s}$ \\
$C_{\theta}$ & pitch structural damping coefficient & $0.18 \mathrm{~m}^{2} \mathrm{~kg} / \mathrm{s}$ \\
$K_{h}$ & plunge structural spring coefficient & $2844.4 \mathrm{~N} / \mathrm{m}$ \\
$C_{1, g}$ & lift coefficient for gurney flap & $18.45 / \mathrm{rad}$ \\
$C_{g, g}$ & moment coefficient for gurney flap & $3.25 \mathrm{rad}$ \\
$K_{h}$ & plunge stiffness & $6.833 \mathrm{~N} \cdot \mathrm{m} / \mathrm{rad}$ \\
\hline \hline
\end{tabular}

In this part, with the governing equations (3.12) and (3.13), we can get the state- 
space equation for this model and it is reasonable to get the numerical value from the Table 1 to get the system parameters. Indeed, we can see that the system parameter includes time-varying parts, which are $C_{1, \theta}$ and $C_{g, \theta}$.

\section{NACA0012 Airfoil State Space Model}

In this part, we present the state space of the NACA0012 airfoil with the generated parameters in Table I.

To begin, the state variables are defined as

$$
x=\left[\begin{array}{l}
h \\
\theta \\
\dot{h} \\
\dot{\theta}
\end{array}\right]
$$

The state space model of the NACA0012 airfoil is written in the from:

$$
\begin{gathered}
\dot{x}=A x+B u \\
y=C x \\
\text { where } A=\left[\begin{array}{cc}
0_{2 \times 2} & I_{2 \times 2} \\
-M^{-1} K & -M^{-1} N
\end{array}\right], B=\left[\begin{array}{c}
0_{2 \times 1} \\
M^{-1} R
\end{array}\right], C=\left[\begin{array}{llll}
0 & 1 & 0 & 0
\end{array}\right]
\end{gathered}
$$


which $M, K, R, C$ matrices are in the following structure:

$$
\begin{aligned}
M & =\left[\begin{array}{cc}
m & m x_{\theta} b \\
m x_{\theta} b & m
\end{array}\right], \\
K & =\left[\begin{array}{cc}
K_{h} & \rho U^{2} b C_{1, \theta} \\
0 & K_{\theta}-\rho U^{2} b^{2} C_{g, \theta}
\end{array}\right], \\
R & =\left[\begin{array}{cc}
\rho U^{2} b C_{1, g} \\
\rho U^{2} b^{2} C_{g, g}
\end{array}\right], \\
N & =\left[\begin{array}{cc}
C_{h}+\rho U b C_{1, \theta} & \rho U b^{2} C_{1, \theta}\left(\frac{1}{2}-a\right) \\
-\rho U b^{2} C_{g, \theta} & C_{\theta}-\rho U b^{3} C_{g, \theta}\left(\frac{1}{2}-a\right)
\end{array}\right]
\end{aligned}
$$

The control input $u=g$, which is considered as gurney flap, and $y=\theta$ denotes the system output.

Based on the existed control design which will be introduced in Chapter 4, we will generate the system matrix parameters in two cases with Table I. First, the blade rotation parameter $C_{1, \theta}$ is constant which means the $\omega_{r}$ part is equal to zero. Second, the blade rotation parameter includes the time-varying parts, which the time frequency $\omega_{r}$ is equal two [3]. 


\section{A nominal model with constant parameters}

In this case, we generate the blade rotation by assuming the $\omega_{r}=0$. Then we can use the existed control design in next chapter.

$$
\begin{aligned}
A_{1} & =\left[\begin{array}{cccc}
0 & 0 & 1.0000 & 0 \\
0 & 0 & 0 & 1.0000 \\
-229.8782 & -2.8663 & -2.5774 & -0.0572 \\
7.5865 & -0.5289 & 0.0761 & -0.0141
\end{array}\right] \\
B & =\left[\begin{array}{cc}
0 \\
0 \\
15.7941 \\
0.8901
\end{array}\right] \\
C & =\left[\begin{array}{lll}
0 & 1 & 0 \\
0
\end{array}\right]
\end{aligned}
$$

\section{An actual model with time-varying parameters}

In this case, we generate the actual model with some time-varying parameters,

which will be applied in Chapter 5. In Chapter 5, we will present our new adaptive control design using this state space model parameters. 


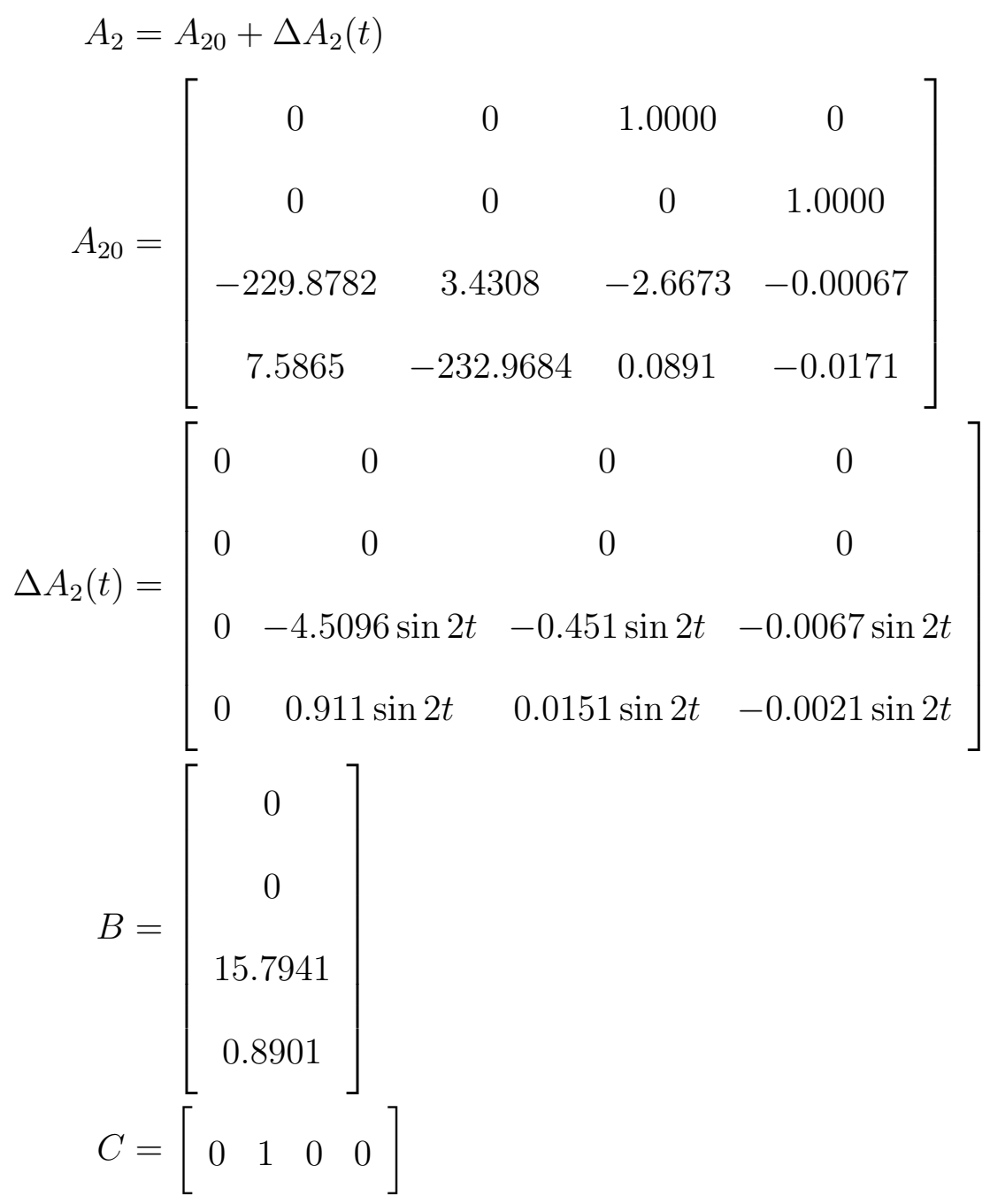

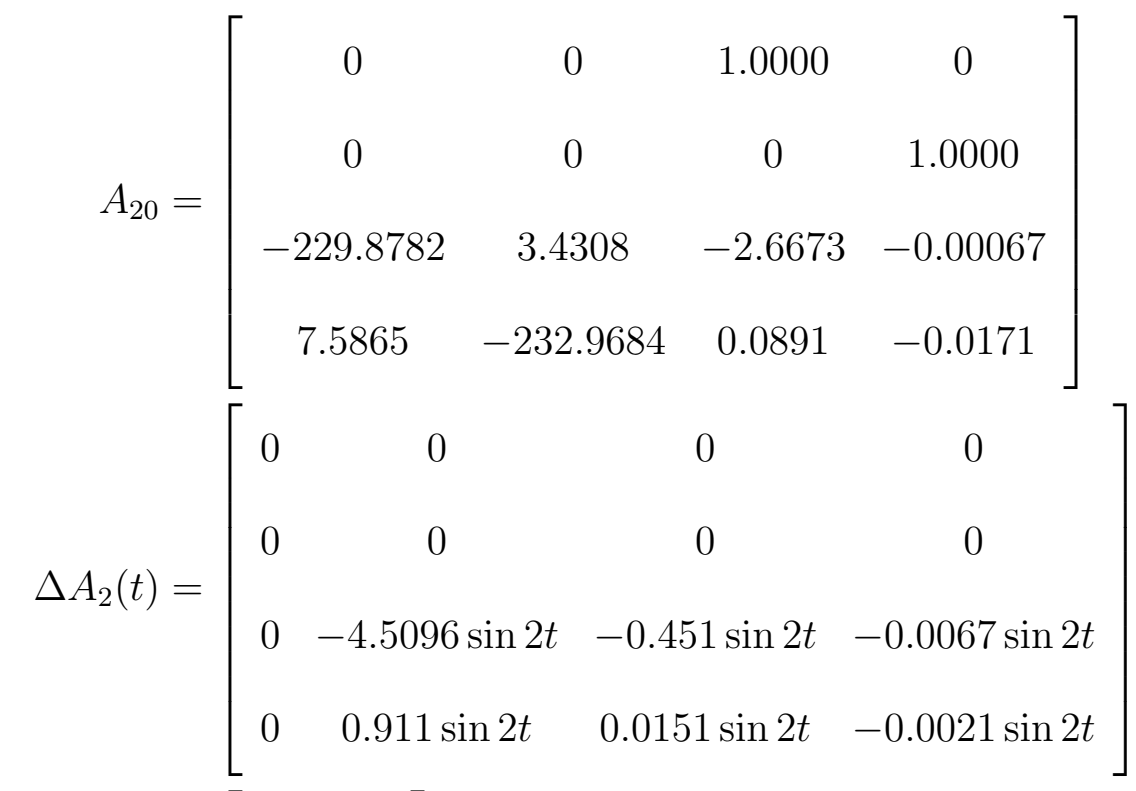

$$
\begin{aligned}
& \begin{array}{l}
B=\left[\begin{array}{c}
0 \\
0 \\
15.7941 \\
0.8901
\end{array}\right] \\
C=\left[\begin{array}{llll}
0 & 1 & 0 & 0
\end{array}\right]
\end{array}
\end{aligned}
$$

From the above parameters we generated, we see that our control matrix and output matrix $B$ and $C$ are the same in two cases, the difference between matrices $A_{1}$ and $A_{2}$ will lead us to the following chapters, we will present our simulation results to see that our new adaptive control design can solve the problem of time-varying system parameters. 


\subsection{Summary}

In this chapter, we discuss the wind turbine systems in several parts. First, some basic components of the wind turbine device are introduce. After that, the physical modeling of the wind turbine is presented in electrical and mechanical part respectively. It is helpful to fully understand the working principle of the main parts in the wind turbine. Then we illustrate a smart material actuator, gurney flap, which supplies the control input in our control design system. And the phenomenon of the hysteresis are talked about in a new perspective of improving the efficiency of the power production of the wind turbine system. But the control design of decrease the effect of hysteresis is not including in this thesis. In last section, we present the dynamic model of the wind turbine systems which is referred as NACA 0012 airfoil model. Furthermore, we also generate the system matrices into two parts, which are the nominal model with constant parameters part and the actual model with time-varying parameters part. We would like to see that whether we can achieve our control objective with these different system matrices by using the existed control design in next chapter. 


\section{Chapter 4}

\section{Study of Adaptive Model Reference Control Designs}

Adaptive state feedback control designs will be applied in this chapter. In this part, we will introduce two adaptive state feedback control designs, which are state tracking and output tracking. Before the algorithms are utilized, we need to check whether our control designs satisfy the assumptions. Then we will present our control design procedure, which includes the controller form, adaptive laws and stability analysis.

\subsection{State Tracking Control Design}

Either state tracking or output tracking is designed to improve the system performances, it is necessary to ensure two prior conditions to be satisfied. First, all signals in the closed-loop system should be bounded. Second, the state vector $x_{p}(t)$ should make an asymptotic tracking to a given reference signal. 


\subsubsection{State Tracking Controller Structure}

Consider a plant described by a differential equation

$$
\dot{x}_{p}(t)=A_{p} x_{p}(t)+b_{p} u(t)
$$

where the system state vector $x_{p} \in R^{n}$, the control input is $u(t) \in R$, with the initial state is $x_{p}(0)=x_{p 0}$. And the system matrix $A_{p} \in R^{n \times n}$, the control input matrix $B_{p} \in R^{n}$ is unknown constant matrix, and the state vector $x_{p}(t)$ is available for measurement.

The control objective is to design a feedback control $u(t)$ that in the closed-loop system all signals are bounded and the state vector $x_{p}(t)$ asymptotically tracks a given a reference state vector $x_{m}(t)$ which can be generated from the reference system

$$
\dot{x}_{m}(t)=A_{m} x_{m}(t)+b_{m} r(t)
$$

with the initial reference state condition $x_{m}(0)=x_{m 0}$, where the reference state vector $x_{m} \in R^{n}$, the reference input is $r(t) \in R$, the reference system matrix $A_{m} \in R^{n \times n}$ and reference input matrix $B_{m} \in R^{n}$ are known and constant.

To achieve the design objective, several assumptions are given by

A4.1 All the eigenvalues of $A_{m}$ are in the open left-half complex plane;

A4.2 $r(t)$ is bounded and piecewise continuous;

A4.3 There exist a constant vector $k_{1}^{*} \in \mathbb{R}^{n}$ and a non-zero constant scalar $k_{2}^{*} \in \mathbb{R}$ such that the following equations are satisfied:

$$
A_{p}+b_{p} k_{1}^{* T}=A_{m}, \quad b_{p} k_{2}^{*}=b_{m}
$$


A4.4 $\operatorname{sign}\left[k_{2}^{*}\right]$, the sign of the parameter $k_{2}^{*}$ is known.

The assumption A4.1 and A4.2 are needed for the stabilization of a well-defined reference system with a bounded output $y_{m}(t)$. Assumption A4.3 is a matching condition if the parameters $A_{p}(t)$ and $b_{p}(t)$ are known, there is a control law that

$$
u(t)=k_{1}^{* T} x_{p}(t)+k_{2}^{*} r(t),
$$

So the control objective can be achieved by

$$
\dot{x}_{p}(t)=A_{p} x_{p}(t)+b_{p}\left(k_{1}^{* T} x_{p}(t)+k_{2}^{*} r(t)\right)=A_{m} x_{m}(t)+b_{m} r(t)
$$

where the system state vector $x_{p}(t)$ is bounded, the defined tracking error $e(t)=$ $x_{p}(t)-x_{m}(t)$ is defined as follows:

$$
\dot{e}(t)=A_{m} e(t)
$$

where the initial condition $e(t)=x_{p 0}-x_{m 0}$, shows that the tracking error goes to zero exponentially.

\subsubsection{Limitations of State Tracking Control Design}

In order to apply the method of state tracking control design, we need to testify the assumptions from last section. After calculating the matching condition (4.3), there is no solution for $k_{1}^{*}$ applying for any models. Since $k_{1}^{*}$ does not exist, adaptive state tracking design is not accessible. There is no ideal controller existing that can generate $A_{p}$ to $A_{m}$. If an ideal controller does not exist for state tracking then an adaptive controller will not exist because there are no possible values for $k_{1}$ and $k_{2}$ for the system to calculate with this configuration. 


\subsection{Output Tracking Control Design}

Due to the limitations of state tracking control design, in this section, we will present the output tracking design to apply the system parameters in Section 3.4, the constant blade rotation part, which will be used in this part. To design an adaptive state feedback model reference controller for generating the system input $u(t)$ which ensures closed-loop signal boundedness and asymptotic tracking of an independent reference signal $y_{m}(t)$ by the system output $y(t)$. Stability properties as well as performance objectives are important to consider in the design process and needy to be examined. First we consider a linear time-invariant state space model like

$$
\dot{x}(t)=A x(t)+B u(t), y(t)=C x(t)
$$

where $x(t) \in R^{n}, u(t) \in R$, and $y(t) \in R$. The matrices $A \in R^{n \times n}, B \in R^{n \times 1}$, and $C \in R^{1 \times n}$. So the transfer function of the system is written in the form:

$$
y(s)=C(s I-A)^{-1} B u(s)=\frac{Z(s)}{P(s)} u(s) .
$$

where $Z(s)=z_{m} s^{m}+z_{m-1} s^{m-1}+\cdots+z_{1} s+z_{0}, z_{m} \neq 0$. The degree of $P(s)$ is $n$.

Matching conditions for output tracking. In order to achieve the control design objective, some assumptions are needed

(A4.5) (A,B,C) is stabilizable and detectable;

(A4.6) $Z(s)$ is a stable polynominal;

(A4.7) Degree of $Z(s), m$ is known;

(A4.8) The sign of $z_{m}$ is known. 
The assumption A4.6 ensures that system output $y(t)$ is going to track the reference system output $y_{m}(t)$. Assumption A4.7 provides a choice of reference model for the adaptive control, meanwhile A4.8 is needed for an adaptive parameter updated law [4]. Furthermore, with (4.8) and above assumptions, the reference model of the nominal model with constant parameters above can be chosen. So the reference model is independent of the dynamics of the system, which is chosen

$$
y_{m}(t)=W_{m}(s)[r](t), W_{m}(s)=\frac{1}{P_{m}(s)} .
$$

where $P_{m}(s)$ is the desired closed-loop characteristic polynomial, its degree is $n-m$.

When $P_{m}(s)$ chosen, we need to guarantee that the ideal gains $K^{*}$ and $k_{r}^{*}$ exist, which can be calculated from the following matching equation:

$$
\operatorname{det}\left(s I-A-B K^{*}\right)=P_{m} Z(s) \frac{1}{z_{m}}, k_{r}^{*}=\frac{1}{z_{m}}
$$

So for the nominal model with constant parameters, we can get the ideal gains from our control input to output,

$$
\begin{aligned}
K^{*} & =\left[\begin{array}{llll}
0.5801 & 5.5046 & -0.2953 & 1.2902
\end{array}\right]^{T}, \\
k_{r}^{*} & =-1.1235 .
\end{aligned}
$$

\subsubsection{Output Tracking Controller Structure}

After we calculate the ideal gains for the suitable case, the fixed version of the adaptive controller is

$$
u(t)=K^{*} x(t)+k_{r}^{*} r(t)
$$


which would lead to the desired closed-loop system: $y(s)=W_{m}(s) r(s)$. Since $A, B$ and $Z_{m}$ are all unknown parameters, the desired control gains $K^{*}$ and $k_{r}^{*}$ are also unknown. So an adaptive law is needed to update parameter estimates $K(t)$ and $k_{r}(t)$. The controller form will become

$$
u(t)=K(t) x(t)+k_{r} u(t)
$$

with the adaptive controller form (4.14), the system (3.15) becomes

$$
\begin{aligned}
\dot{x}(t) & =A x(t)+B\left(K(t) x(t)+k_{r}(t) r(t)\right) \\
& =\left(A+B K^{*}\right) x(t)+B k_{r}^{*}(t)+B\left(\left(K(t)-K^{*}\right) x(t)+\left(k_{r}(t)-k_{r}^{*}\right) r(t)\right), \\
y(t) & =C x(t) .
\end{aligned}
$$

\subsubsection{Adaptive Law Design}

From (4.9), it follows that

$$
C\left(s I-A-B K^{*}\right)^{-1} B k_{r}^{*}=\frac{Z(S) k_{r}^{*}}{\operatorname{det}\left(s I-A-B K^{*}\right)}=\frac{1}{P_{m}(s)}=W_{m}(s) .
$$

In view of (4.9), (4.15) and (4.16), the closed-loop tracking error equation becomes

$$
e(t)=y(t)-y_{m}(t)=\rho^{*} W_{m}(s)\left[\left(K-K^{*}\right) x+\left(k_{r}-k_{r}^{*}\right) r\right](t)+C e^{\left(A+B K^{*}\right) t} x(0) .
$$

where $\rho^{*}=z_{m}$, and $C e^{\left(A+B K^{*}\right) t} x(0)$ will go to zero exponentially. We note that the reference output $y_{m}(t)=W_{m}(t)[r](t)$ is derived from an $(n-m)$ th order state variable equation which depends on the parameters of $W_{m}(s)$ only and is defined to have zero initial conditions. 
Estimation error. In order to get the estimation error equation, we introduce several elements as follows:

$$
\begin{aligned}
\theta(t) & =\left(K(t), k_{r}(t)\right)^{T}, \\
\theta^{*} & =\left(K^{*}, k_{r}^{*}\right)^{T}, \\
\omega(t) & =\left(x^{T}(t), r(t)\right)^{T}, \\
\zeta(t) & =W_{m}(s)[\omega](t), \\
\xi(t) & =\theta^{T}(t) \zeta(t)-W_{m}(s)\left[\theta^{T} \omega\right](t) .
\end{aligned}
$$

and the estimation error is

$$
\epsilon(t)=e(t)+\rho(t) \xi(t)
$$

where $\rho(t)$ is an estimate of $\rho^{*}=z_{m}$. Then, substituting (4.18)-(4.22) in (4.24), and ignoring the initial part of $C e^{\left(A+B K^{*}\right) t} x(0)$, we have

$$
\epsilon(t)=\rho^{*}\left(\theta(t)-\theta^{*}\right)^{T} \zeta(t)+\left(\rho(t)-\rho^{*}\right) \xi(t)
$$

Adaptive laws. In order to determine the adaptive laws to update the estimated parameters $\theta(t)$ and $\rho(t)$, the normalized gradient algorithm will be used [4]. So this error equation suggests the following adaptive laws

$$
\begin{aligned}
& \dot{\theta}(t)=-\frac{\Gamma \operatorname{sign}\left(z_{m}\right) \zeta(t) \epsilon(t)}{1+\zeta^{T}(t) \zeta(t)+\xi^{2}(t)}, \\
& \dot{\rho}(t)=-\frac{\gamma \xi(t) \epsilon(t)}{1+\zeta^{T}(t) \zeta(t)+\xi^{2}(t)} .
\end{aligned}
$$


where $\operatorname{sign}\left(z_{m}\right)$ is the sign of the parameter $z_{m}, \Gamma=\Gamma^{T}>0$ and $\gamma>0$ are adaption gains. And the signal $m(t)$ is such that

$$
m(t)=\sqrt{1+\zeta^{T}(t) \zeta(t)+\xi^{2}(t)}
$$

where the boundedness of $\frac{\epsilon(t)}{m(t)}$ is ensured when $\theta(t)$ and $\rho(t)$ are bounded [4].

\subsubsection{Stability Analysis}

We employ a Lyapunov candidate function to generate some properties which bases on the adaptive output tracking design, consider a positive definite function

$$
V(\tilde{\theta}, \tilde{\rho})=\frac{1}{2}\left(\left|\rho^{*}\right| \tilde{\theta}^{T} \Gamma^{-1} \tilde{\theta}+\gamma^{-1} \tilde{\rho}^{2}\right)
$$

where

$$
\tilde{\theta}(t)=\theta(t)-\theta^{*}, \tilde{\rho}(t)=\rho(t)-\rho^{*}
$$

The time derivative of (4.28), with respect of (4.25) and (4.26), is to be

$$
\dot{V}=-\frac{\epsilon^{2}(t)}{m^{2}(t)} \text {. }
$$

which we can get that (4.30) is negative definite, the adaptive law (4.25) and (4.26) guarantees that $\theta(t) \in L^{\infty}, \rho(t) \in L^{\infty}, \frac{\epsilon(t)}{m(t)} \in L^{2} \cap L^{\infty}, \dot{\theta}(t) \in L^{2} \cap L^{\infty}$, and $\dot{\rho}(t) \in$ $L^{2} \cap L^{\infty}[4]$.

With the above properties obtained from the stability analysis, all signals in the closed-loop system are bounded. Therefore, the tracking error, e(t), satisfies the 
following properties

$$
\begin{gathered}
\lim _{t \rightarrow \infty}\left(y(t)-y_{m}(t)\right)=0 \\
\int_{0}^{\infty}\left(y(t)-y_{m}(t)\right)^{2} d t<\infty .
\end{gathered}
$$

\subsubsection{Simulation Study}

\section{Simulation conditions}

We present our simulation work in several scenarios. With (4.11) and (4.12), we present the results of the nominal model with constant parameters part in fixed version of (4.13). Fixed gain controllers were simulated to compare their performance to that of the designed adaptive controllers. In order to show our simulation results, we need some initial conditions, the reference input is chosen as $r(t)=1$.

\section{Simulation Results}

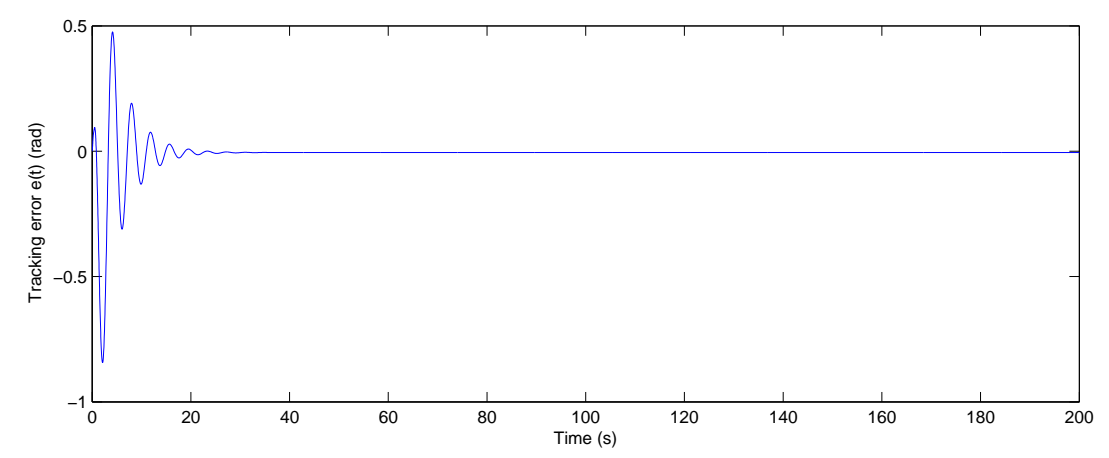

Figure 4.1: Tracking error (Scenario I: nominal control, $\omega_{r}=0$ )

Next, we present the simulation results when we apply the designed adaptive laws. In this scenario, the initial conditions will change, which will be chosen as the $75 \%$ of (4.11) and (4.12). 


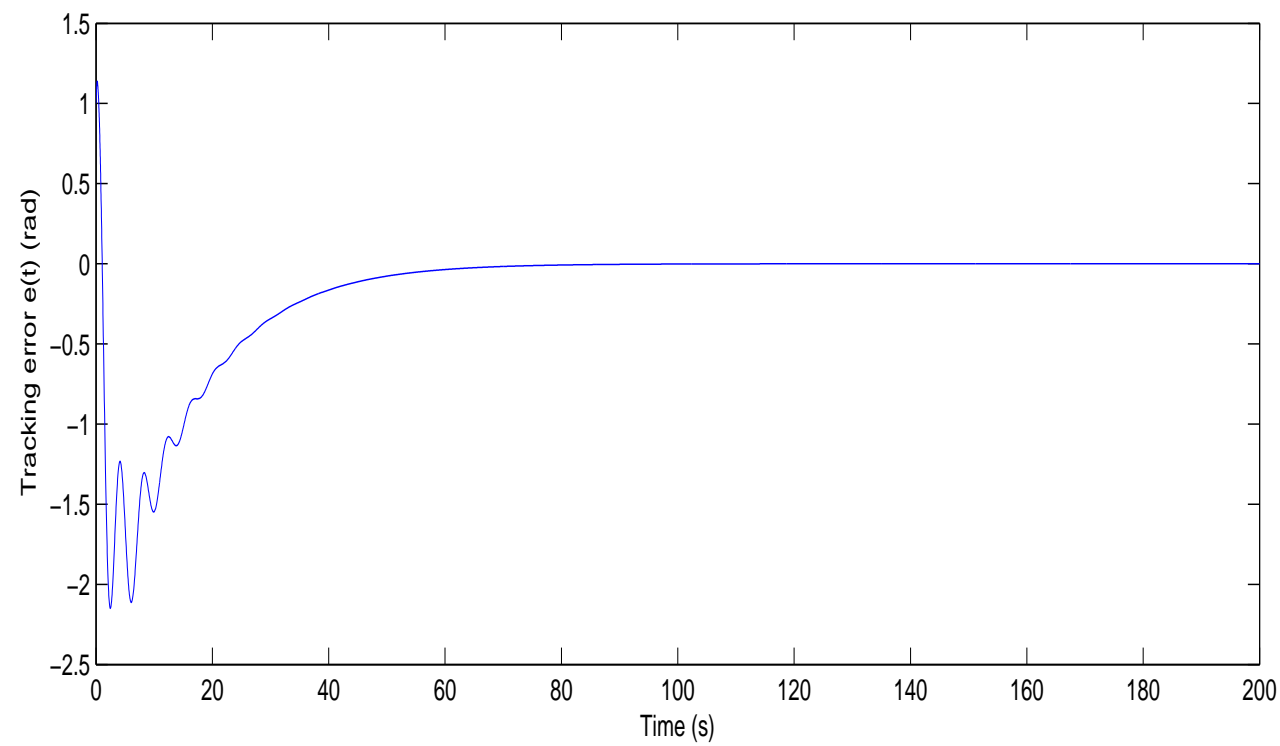

Figure 4.2: Tracking error (Scenario II: adaptive control, $\theta(0)=75 \% \theta^{*}, \Gamma=5$ )
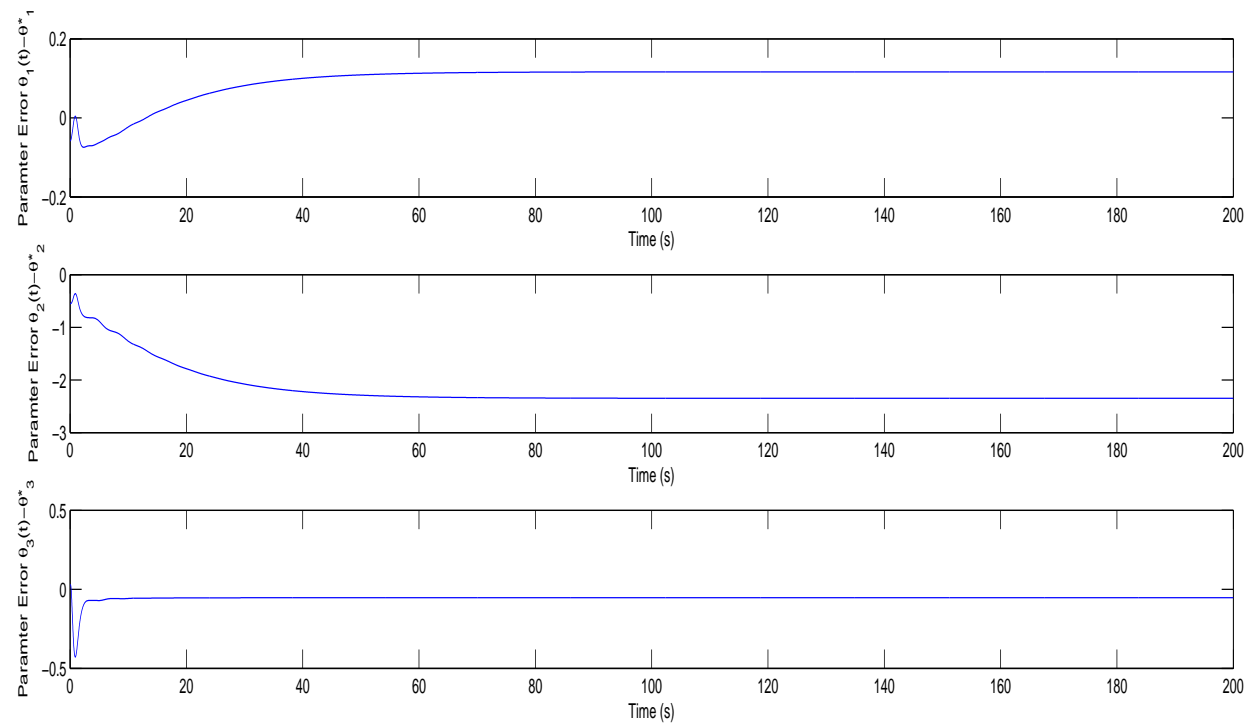

Figure 4.3: Parameter errors for $\theta_{1}, \theta_{2}, \theta_{3}$, when $\theta(0)=75 \% \theta^{*}$ 

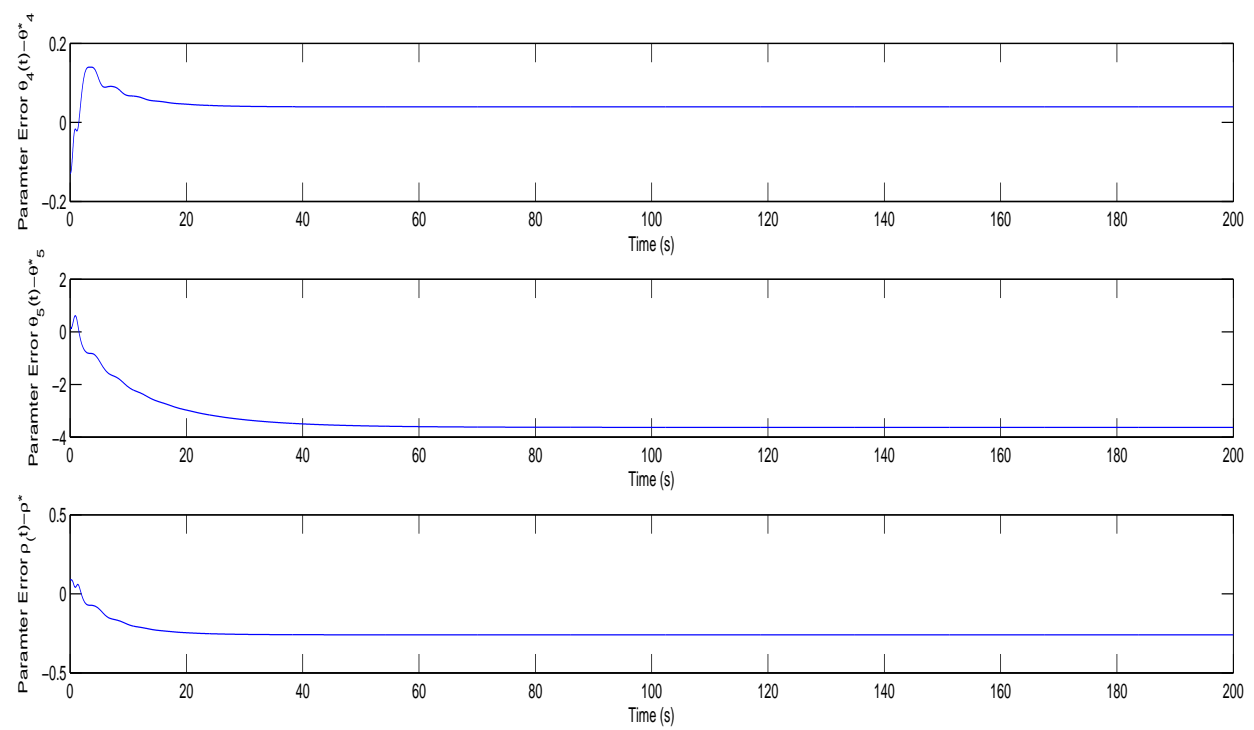

Figure 4.4: Parameter errors for $\theta_{4}, \theta_{5}, \rho$, when $\theta(0)=75 \% \theta^{*}$

The results show that in the case of the nominal model with constant parameters part, the output tracking control design can achieve the objective that the output pitch angle $\theta$ can follow the reference signal which is chosen to satisfy the physical meaning. However, what about the case the actual model with time-varying parameters part by using the output tracking control design? 


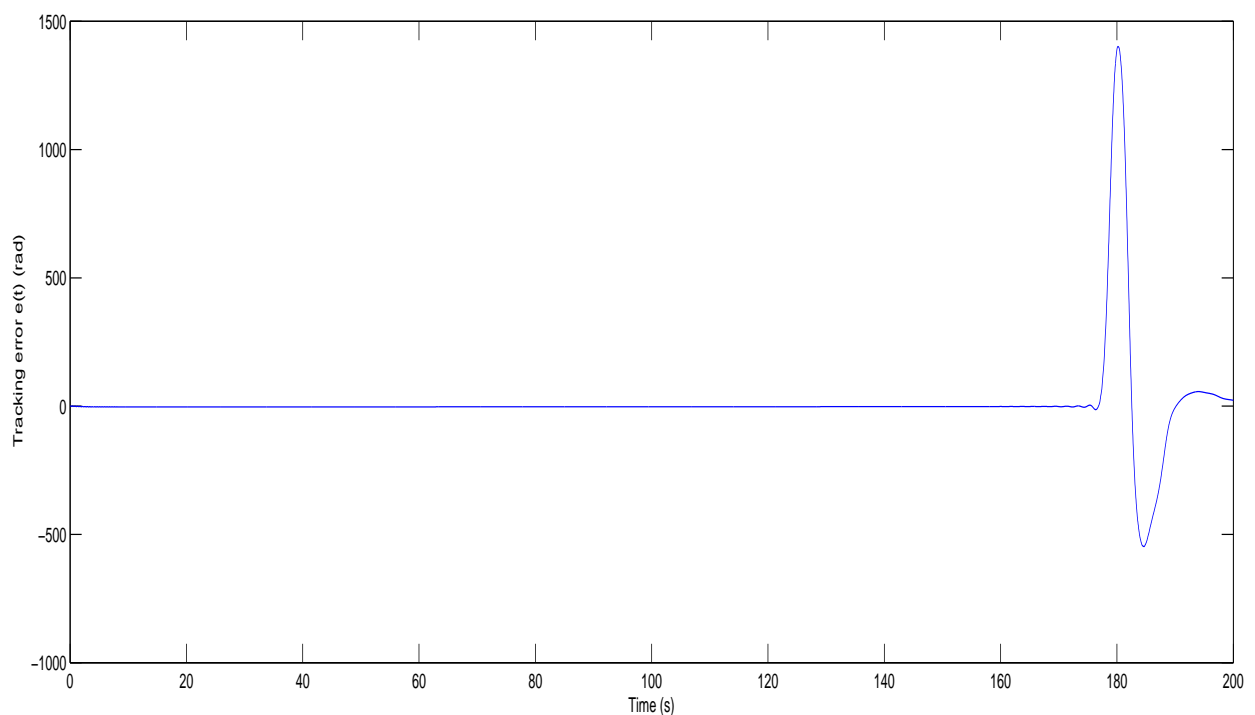

Figure 4.5: Scenario III: nominal control, $\omega_{r}=2$

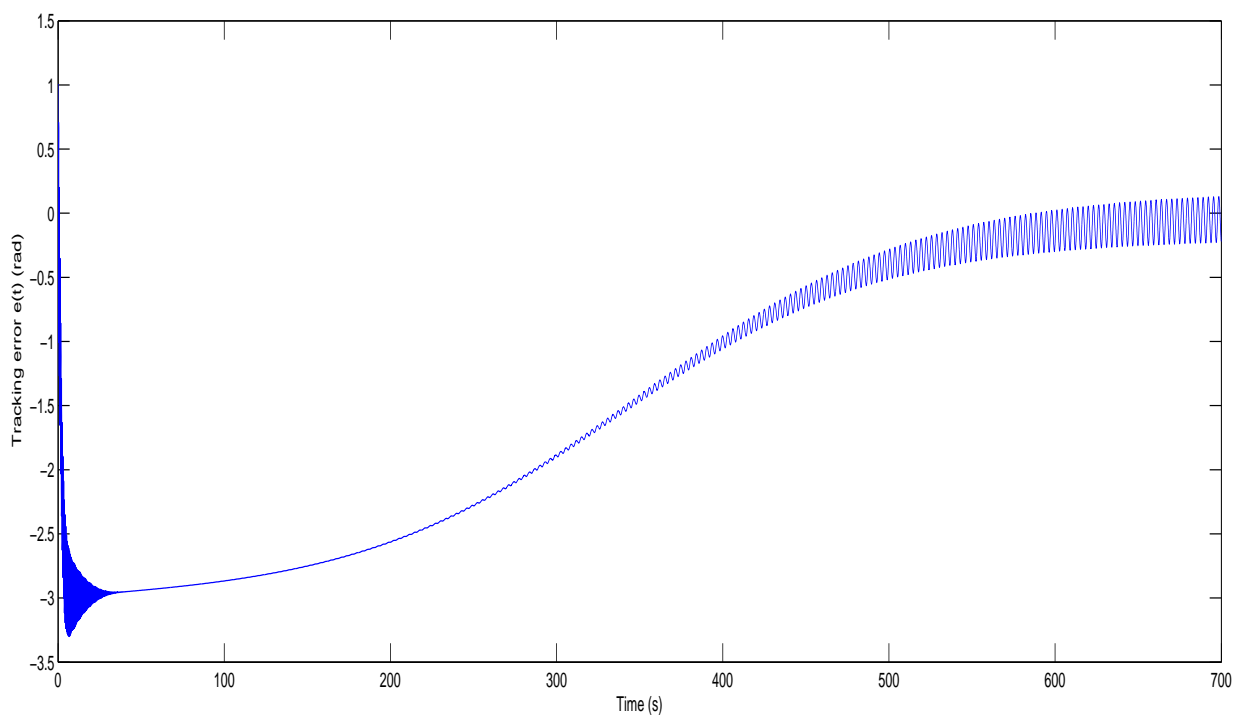

Figure 4.6: Tracking error (Scenario IV: adaptive control, $\omega_{r}=2, \Gamma_{1}=0.5$ ) 

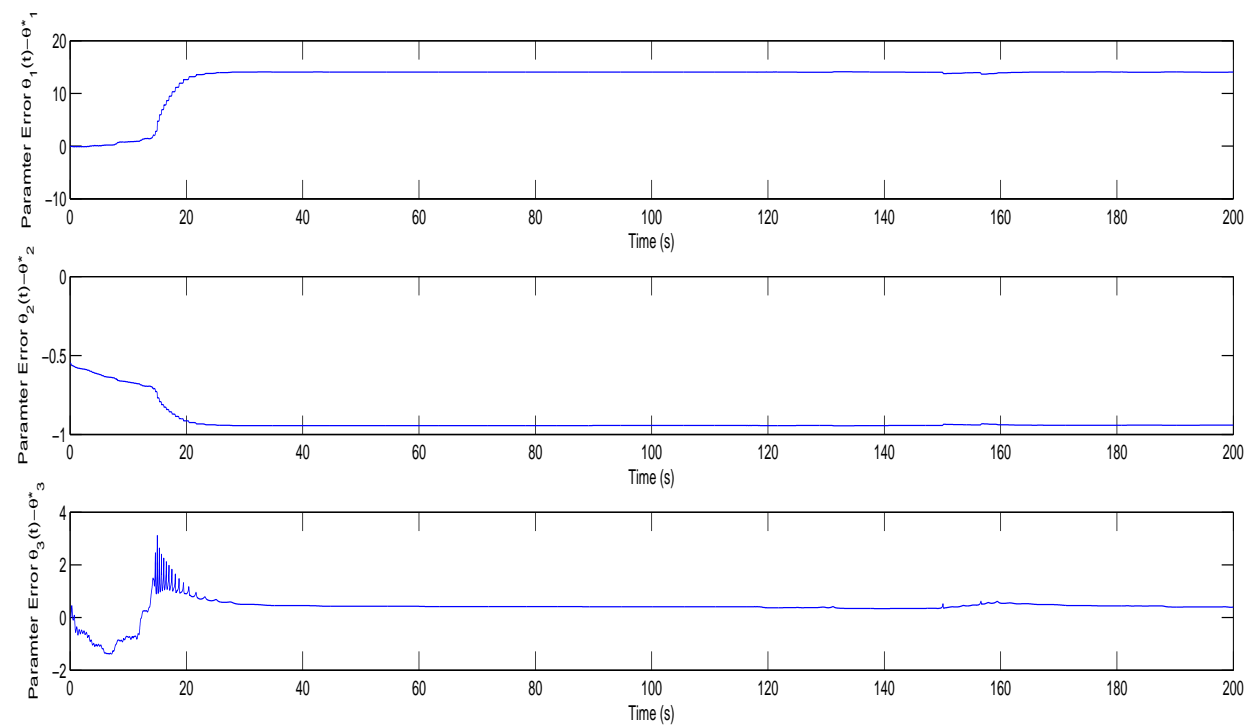

Figure 4.7: Parameter errors for $\theta_{1}, \theta_{2}, \theta_{3}, \theta(0)=90 \% \theta^{*}$
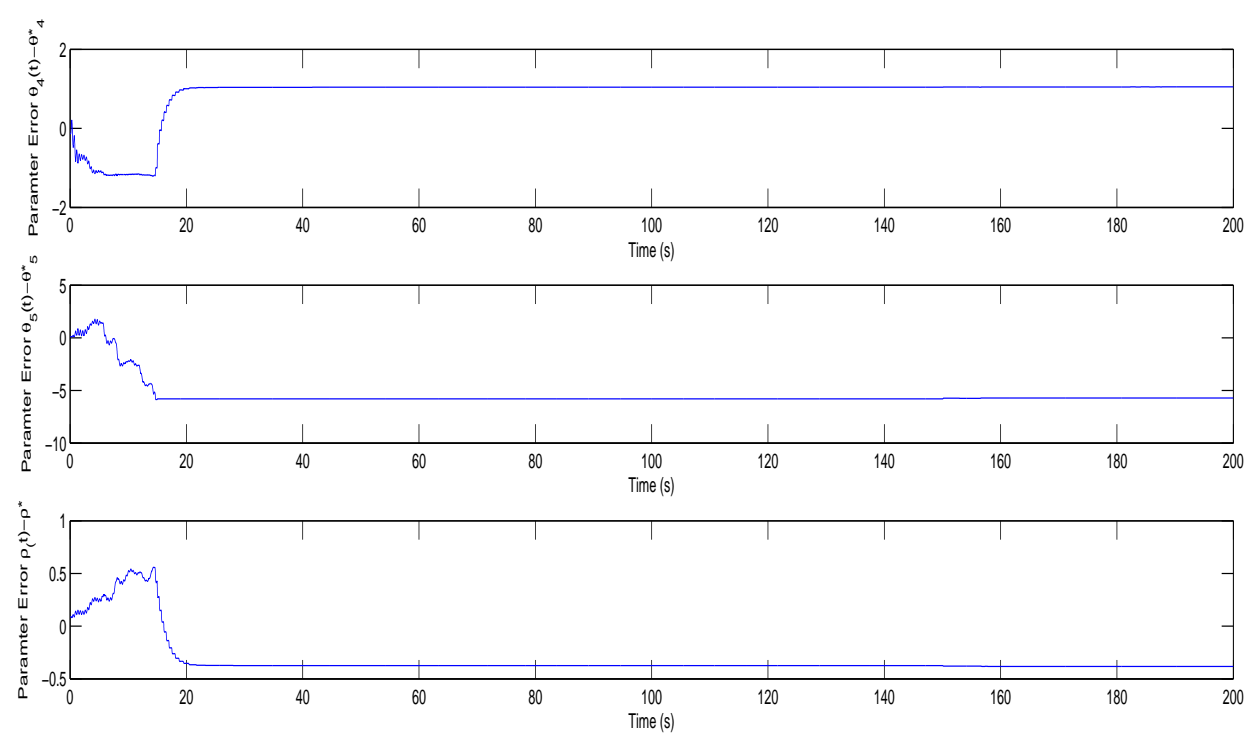

Figure 4.8: Parameter errors for $\theta_{4}, \theta_{5}, \rho, \theta(0)=90 \% \theta^{*}$ 


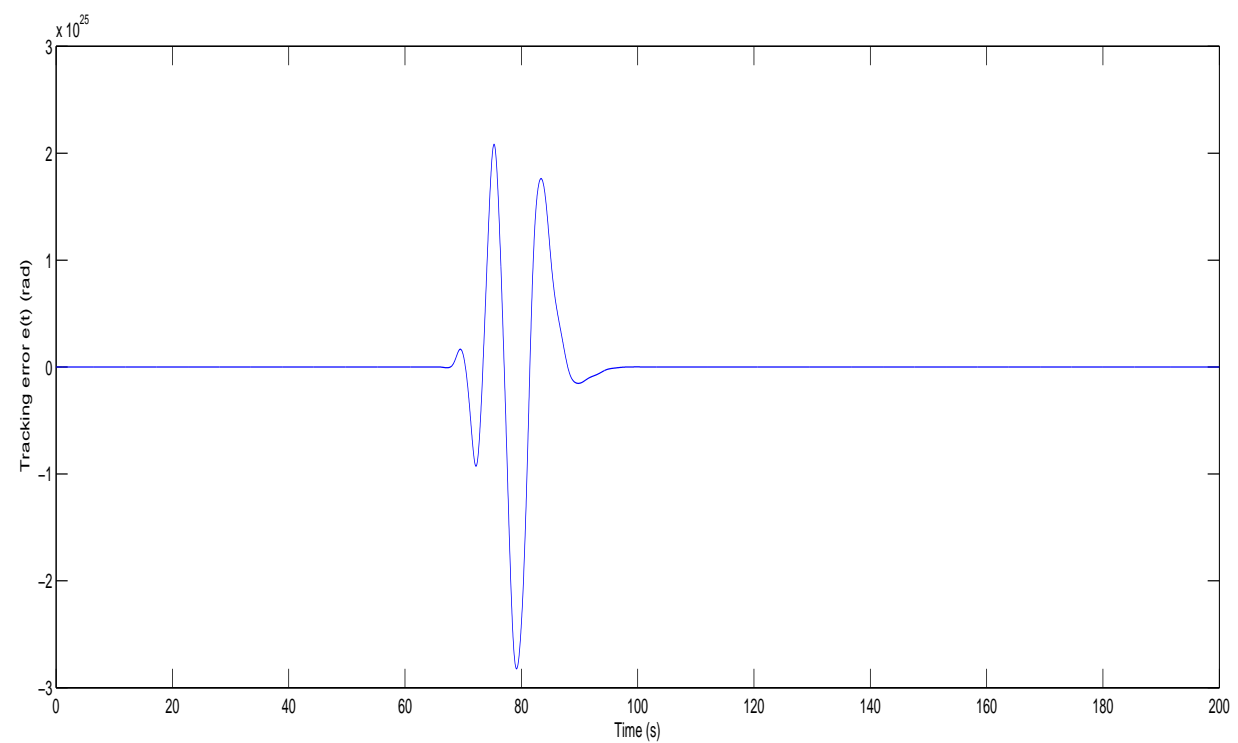

Figure 4.9: Tracking error (Scenario V: adaptive control, $\omega_{r}=2, \Gamma_{2}=5$ )

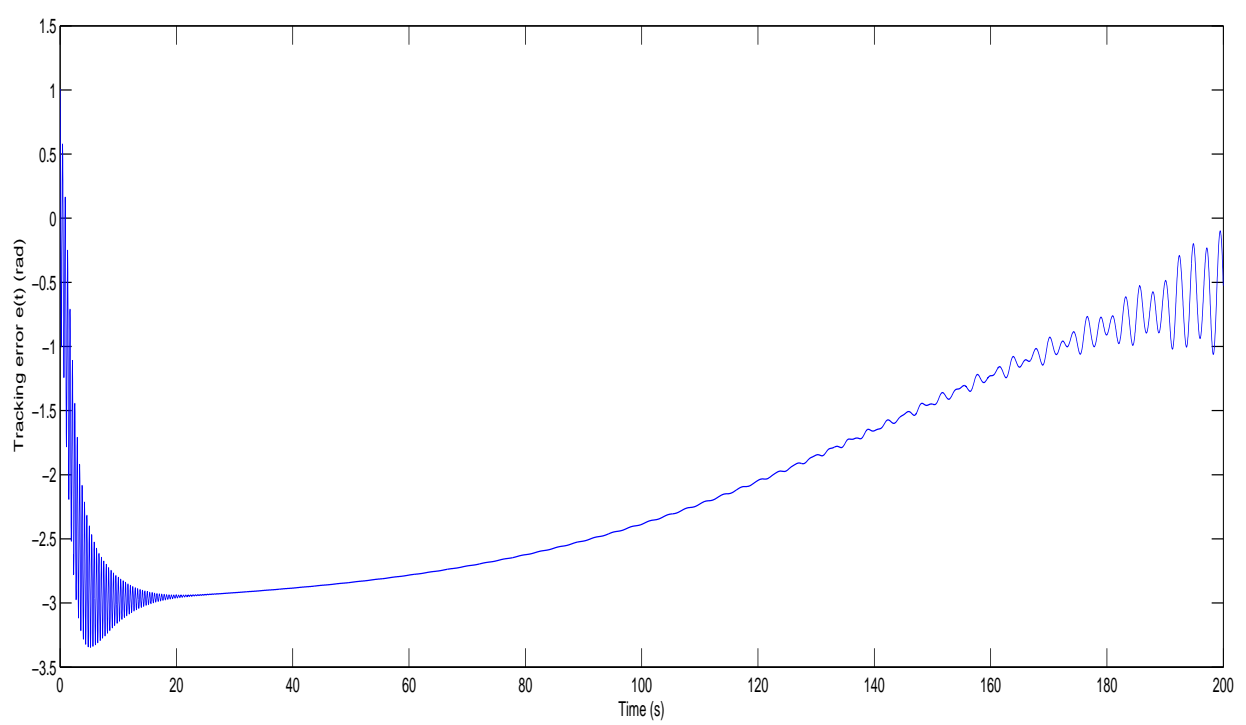

Figure 4.10: Tracking error (Scenario VI: adaptive control, $\omega_{r}=2, \Gamma_{3}=15$ )

From simulation results, the adaptive output tracking design is not appropriate for the case of the actual model with time-varying parameters part. In next chapter, we will present the adaptive backstepping control design to fix this problem. 


\section{Chapter 5}

\section{Adaptive Backstepping Control}

In this chapter, adaptive backstepping control design will be introduced based on the specific case of the state space equation of the wind turbine systems. In our adaptive output tracking design, the controllers designed employ static strict feedback, the controllers will, in addition, present a form of nonlinear integral feedback. It is a way to deal with uncertain time-varying systems with some unknown bounded disturbances. In this specific case, we don't consider the disturbance terms, but need to deal with the nonlinear part in the control design procedure. It is necessary to use a well-defined control design scheme. The adaptive backstepping control design can overcome the control problems, such as over-parametrization, which means there are more estimated unknown parameters. So this recursive design procedure from parametric strict state feedback will be developed. 


\subsection{A Basic Adaptive Backstepping Scheme}

First, we present a backstepping control design procedure for a second-order nonlinear system in a standard form, which is given by

$$
\begin{aligned}
& \dot{w}_{1}=w_{2} \\
& \dot{w}_{2}=\varphi_{0}\left(w_{1}, w_{2}\right)+\theta^{* T}(t) \varphi_{1}\left(w_{1}, w_{2}\right)+b_{0} u, y=w_{1} .
\end{aligned}
$$

where $\varphi_{i}, i=0,1$, are known functions which are differentiable with respect to $w_{1}, w_{2}$, $b_{0}$ is unknown constant and $\theta^{*}$ is an unknown parameter vector with some known time-varying part, which is chosen

$$
\theta^{*}(t)=\theta_{0}^{*}+\theta_{1}^{*} \sin \omega_{r} t
$$

where $\theta_{0}^{*} \in R^{n_{\theta}}$ and $\theta_{1}^{*} \in R^{n_{\theta}}$ are unknown constant parameter vectors, the time frequency $\omega_{r}=2$ is referred to the blade rotation, it is for the case of the actual model with time-varying parameters.

The control objective is to design a state feedback control $u(t)$ using $w_{1}$ and $w_{2}$, such that all closed-loop signals are bounded and the system output $y(t)$ tracks a given reference output $y_{m}(t)$ asymptotically, where $y_{m}(t), \dot{y}_{m}(t)$, and $\ddot{y}_{m}(t)$ are all bounded functions of time $t$.

\subsubsection{Adaptive Backstepping Control Design}

The following procedure is for the adaptive control scheme of backstepping designed for this basic case, assuming that $b_{0}$ is unknown.

Step 1: Introducing $z_{1}=w_{1}-y_{m}$ and $z_{2}=w_{2}-\alpha_{1}$, where $\alpha_{1}$ is a design function 
to be determined, and the $z_{1}$ equation is chosen

$$
\dot{z}_{1}=\dot{w}_{1}-\dot{y}_{m}=z_{2}+\alpha_{1}-\dot{y}_{m}
$$

At this step, (5.3) can be viewed as a first-order system to be stabilized by $\alpha_{1}$ with respect to the error measure

$$
V_{1}=\frac{1}{2} z_{1}^{2}
$$

The time-derivative of $V_{1}$ is

$$
\dot{V}_{1}=z_{1}\left(z_{2}+\alpha_{1}-\dot{y}_{m}\right)
$$

Choosing the stabilizing function as follows:

$$
\alpha_{1}=-c_{1} z_{1}+\dot{y}_{m}
$$

where $c_{1}>0$ and from (5.3) and (5.5),

$$
\begin{aligned}
& \dot{z}_{1}=z_{2}-c_{1} z_{1} \\
& \dot{V}_{1}=-c_{1} z_{1}^{2}+z_{1}^{T} z_{2}
\end{aligned}
$$

Step 2: Using (5.1) and $z_{2}=w_{2}-\alpha_{1}$,

$$
\begin{aligned}
\dot{z}_{2} & =\dot{w}_{2}-\dot{\alpha}_{1} \\
& =\varphi_{0}\left(w_{1}, w_{2}\right)+\theta^{* T} \varphi_{1}\left(w_{1}, w_{2}\right)+b_{0} u-\dot{\alpha}_{1}
\end{aligned}
$$

Now, (5.3) and (5.9) can be viewed to be stabilized by $\alpha_{1}$ given in (5.6) with respect to the Lyapunov function $V_{2}=V_{1}+\frac{1}{2} z_{2}^{2}+\frac{1}{2}\left(\theta-\theta^{*}\right) \Gamma^{-1}\left(\theta-\theta^{*}\right)+\frac{\left|b_{0}\right|}{2 \gamma} \tilde{\rho}^{2}$, where 
$\Gamma=\Gamma^{T}>0, \gamma$ is a positive constant, $\theta(t)$ is the estimate of $\theta^{*}$, respectively, and $\tilde{\rho}=\hat{\rho}-\rho, \hat{\rho}$ is the estimate of $\rho=\frac{1}{b_{0}}$.

So the time derivative of $V_{2}$ is

$$
\begin{aligned}
\dot{V}_{2}= & -c_{1} z_{1}^{2}+z_{1} z_{2}+z_{2} \dot{z}_{2}+\left(\theta-\theta^{*}\right) \Gamma^{-1}\left(\dot{\theta}-\dot{\theta}^{*}\right)+\frac{\left|b_{0}\right|}{\gamma} \tilde{\rho} \dot{\hat{\rho}} \\
= & -c_{1} z_{1}^{2}+z_{2}\left(w_{1}-y_{m}+\varphi_{0}\left(w_{1}, w_{2}\right)+\theta \varphi_{1}\left(w_{1}, w_{2}\right)+b_{0} u+c_{1}\left(\dot{w}_{1}-\dot{y}_{m}\right)-\ddot{y}_{m}\right) \\
& +\left(\theta-\theta^{*}\right) \Gamma^{-1}\left(\dot{\theta}-\Gamma z_{2} \varphi_{1}\left(w_{1}, w_{2}\right)\right)+\frac{\left|b_{0}\right|}{\gamma} \tilde{\rho} \dot{\hat{\rho}}
\end{aligned}
$$

Choose adaptive laws for $\theta(t)$ as

$$
\dot{\theta}=\Gamma z_{2} \varphi_{1}\left(w_{1}, w_{2}\right)
$$

Then the derivative of $V_{2}$ can be expressed as

$$
\begin{aligned}
\dot{V}_{2} & =-c_{1} z_{1}^{2}+z_{2}\left(b_{0} u-\chi(t)\right)+\frac{\left|b_{0}\right|}{\gamma} \tilde{\rho} \dot{\hat{\rho}} \\
& =-c_{1} z_{1}^{2}+z_{2} b_{0}\left(u-\rho \chi+\rho c_{2} z_{2}-\rho c_{2} z_{2}\right)+\frac{\left|b_{0}\right|}{\gamma} \tilde{\rho} \dot{\hat{\rho}} \\
& =-c_{1} z_{1}^{2}+z_{2} b_{0}(\hat{\rho}-\rho)\left(\chi(t)-c_{2} z_{2}\right)-c_{2} z_{2}^{2}+\frac{\left|b_{0}\right|}{\gamma} \tilde{\rho} \dot{\hat{\rho}}
\end{aligned}
$$

where $c_{2}>0$ and $\chi(t)=-\left(w_{1}-y_{m}+\varphi_{0}\left(w_{1}, w_{2}\right)+\theta \varphi_{1}\left(w_{1}, w_{2}\right)+c_{1}\left(\dot{w}_{1}-\dot{y}_{m}\right)-\ddot{y}_{m}\right)$.

Adaptive backstepping controller. Since this system is nonlinear, the form of controller $u(t)$ is also nonlinear and it should cancel the effect of the time-varying part, so to be stabilized, the controller $u(t)$ as

$$
u(t)=\hat{\rho}\left(\chi(t)-c_{2} z_{2}\right)
$$


Then choose adaptive laws for $\hat{\rho}(t)$

$$
\dot{\hat{\rho}}=-\gamma \operatorname{sign}\left(b_{0}\right) z_{2}\left(\chi(t)-c_{2} z_{2}\right)
$$

Then we can rewrite $\dot{V}_{2}$ as

$$
\dot{V}_{2}=-c_{1} z_{1}^{2}-c_{2} z_{2}^{2}
$$

Stability analysis. After generating the desired result of derivative of the $V_{2}$ function, we will present the stability analysis in this part. Since $\dot{V}_{2} \leq 0$, the error measure $V_{2}=\frac{1}{2} z_{1}^{2}+\frac{1}{2} z_{2}^{2}+\left(\theta-\theta^{*}\right) \Gamma^{-1}\left(\theta-\theta^{*}\right)+\frac{\left|b_{0}\right|}{2 \gamma} \tilde{\rho}^{2}$ does not increase as a function of $t$, that is, $z_{1}(t)=w_{1}(t)-y_{m}(t), z_{2}(t)=w_{2}(t)-\alpha_{1}(t)$ and $\theta(t)-\theta^{*}$ are bounded, and so are $x_{1}(t)$ and $\theta(t)$. From (5.6), $\alpha_{1}(t)$ is bounded, so is $x_{2}(t)$. From the version of $u(t)$ form in (5.13), it shows that $u(t)$ is also bounded. So that all signals in the closed-loop system are bounded.

To generate the tracking measurement, that

$$
c_{1} \int_{0}^{t} z_{1}^{2}(\tau) d \tau+c_{2} \int_{0}^{t} z_{2}^{2}(\tau) d \tau=V_{2}(0)-V_{2}(t)
$$

Since $V_{2}$ as a function of $t$ is bounded, it says that $\int_{0}^{\infty} z_{1}^{2}(\tau) d \tau<\infty$ and $\int_{0}^{\infty} z_{2}^{2}(\tau) d \tau<$ $\infty$. In (5.3), $\dot{z}_{1}(t)$ is bounded, so it shows that $\lim _{t \rightarrow \infty} z_{1}(t)=0$, it means that $\lim _{t \rightarrow \infty}\left(y(t)-y_{m}(t)\right)=0$.

In summary, we have the following conclusion.

Theorem 5.1 The controller in (5.13) with adaptive laws (5.11) and (5.14) applied to the chosen system guarantees that all closed-loop signals are bounded and the tracking error $e(t)=y(t)-y_{m}(t)$ goes to zero when t goes to infinity. 


\subsubsection{Simulation Study}

\section{Simulation conditions}

In this part, we will do the simulation work for a basic adaptive backstepping control design. In our simulation process, $\varphi_{0}\left(w_{1}, w_{2}\right)=w_{1}$ and $\varphi_{1}\left(w_{1}, w_{2}\right)=w_{2}$. From the simulation study, we would like to present that the basic adaptive backstepping control design can deal with the time-varying parameters in our system, in order to so we assume that $\theta_{0}^{*}=2.5, \theta_{1}^{*}=2.5, b_{0}=5$. In different scenarios, we present several results when the reference signals are 1 and $\sin \left(\omega_{p} t\right)$, which $\omega_{p}=1,0.5$. First, we would like to present the simulation result of the nominal control design case, which uses the values we choose, then we will show our adaptive control design simulation results to see that the basic adaptive backstepping control design can achieve the control objective which our system output can track the desired reference signals.

\section{Simulation results}

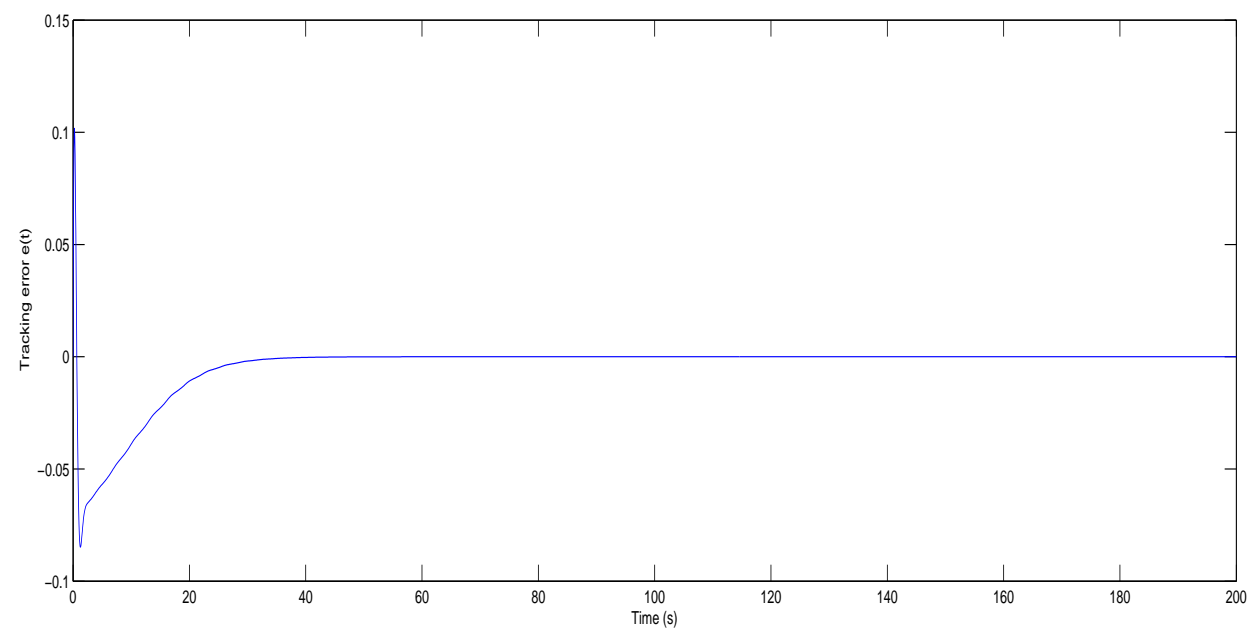

Figure 5.1: Tracking error (nominal control, $\omega_{r}=2, y_{m}=1, \theta(0)=\theta^{*}, \Gamma=0$ ) 


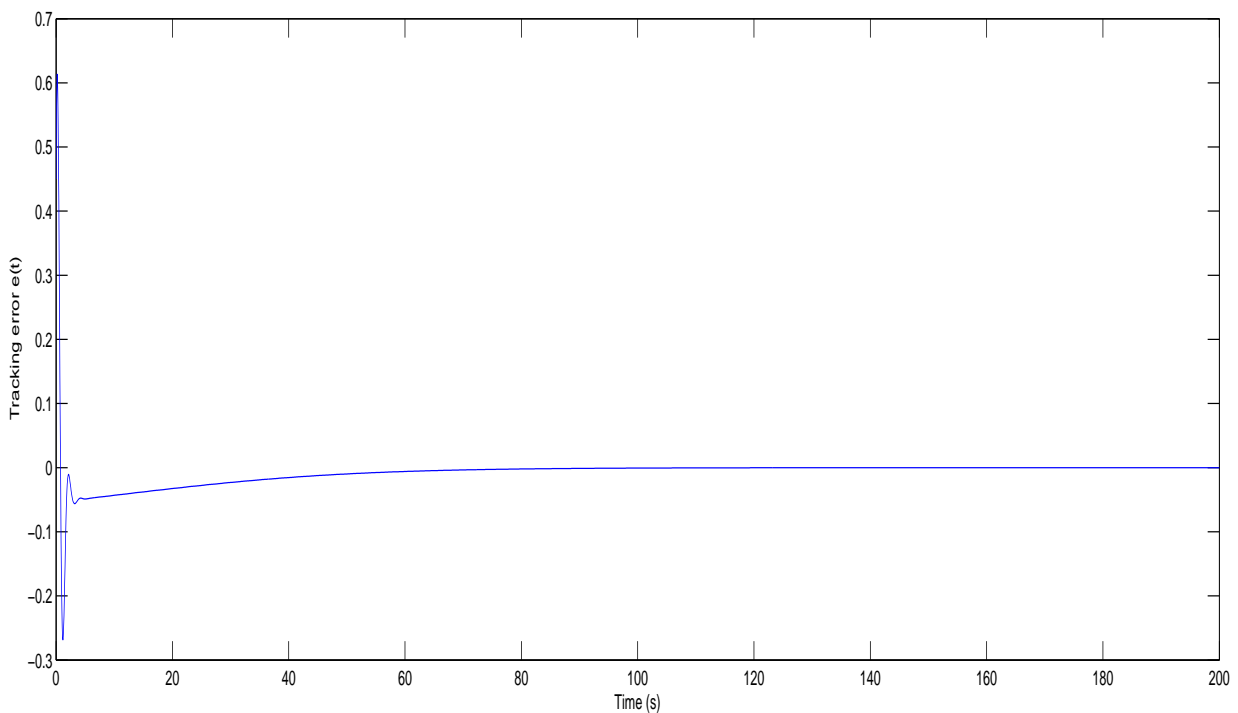

Figure 5.2: Tracking error (adaptive design, $\omega_{r}=2, y_{m}=1, \theta(0)=75 \% \theta^{*}, \Gamma=5$ )

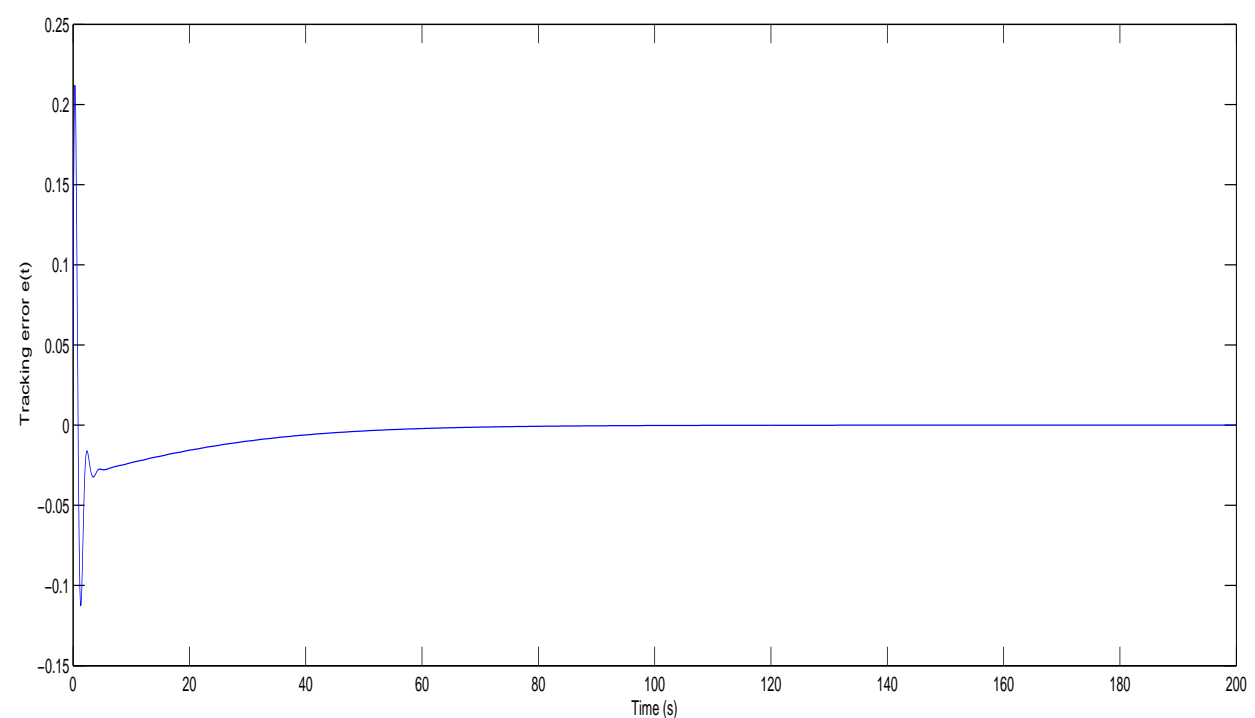

Figure 5.3: Tracking error $\left(\omega_{r}=2, y_{m}=1, \theta(0)=85 \% \theta^{*}, \Gamma=5\right)$ 


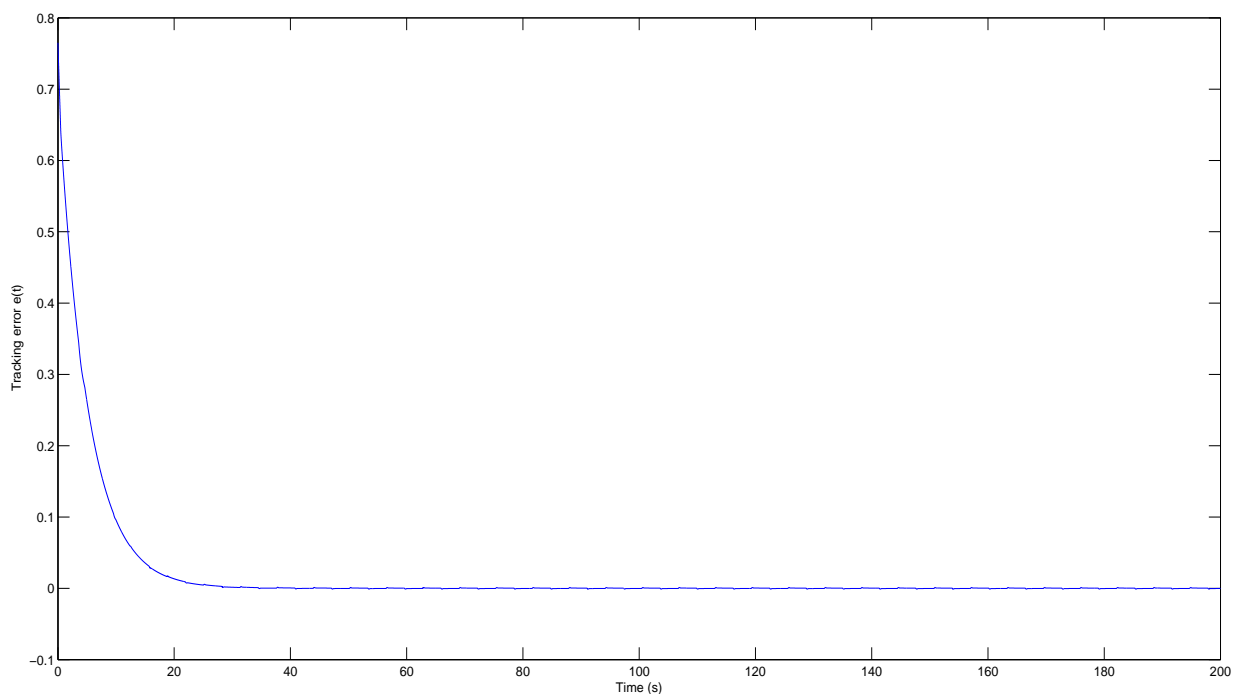

Figure 5.4: Tracking error $\left(\omega_{r}=2, y_{m}=\sin (t), \theta(0)=\theta^{*}, \Gamma=0\right)$

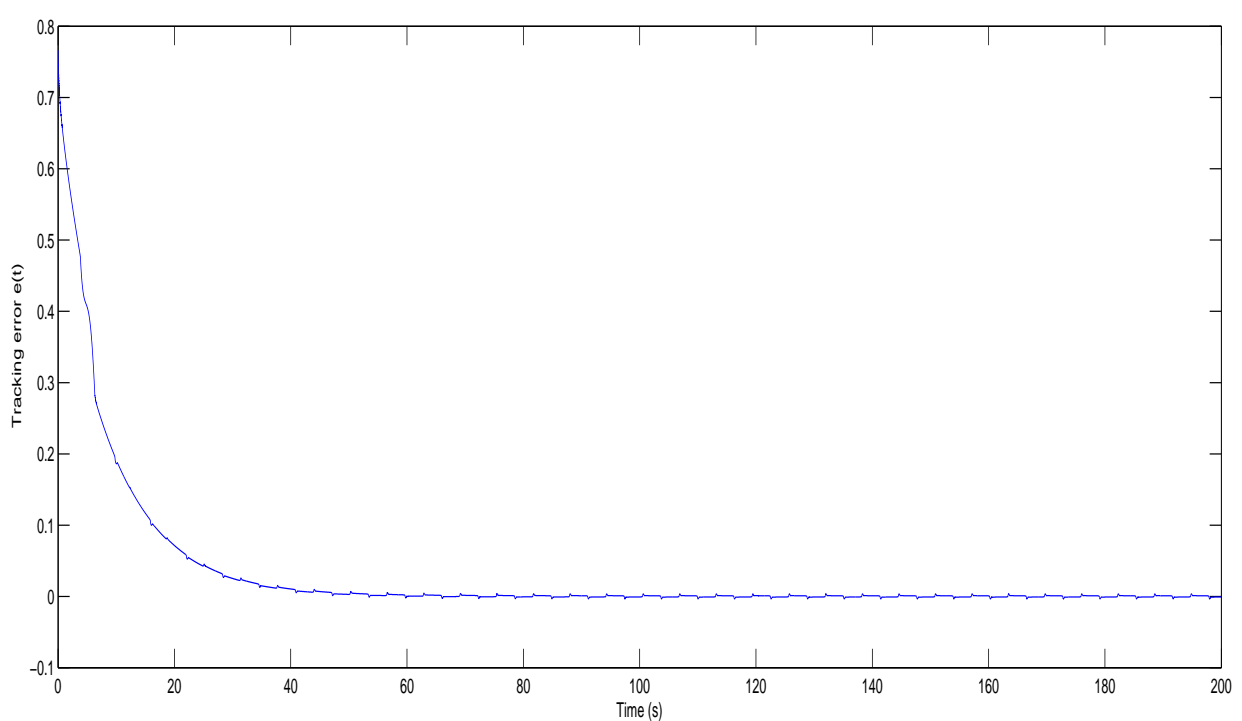

Figure 5.5: Tracking error $\left(\omega_{r}=2, y_{m}=\sin (t), \theta(0)=75 \% \theta^{*}, \Gamma=15\right)$ 


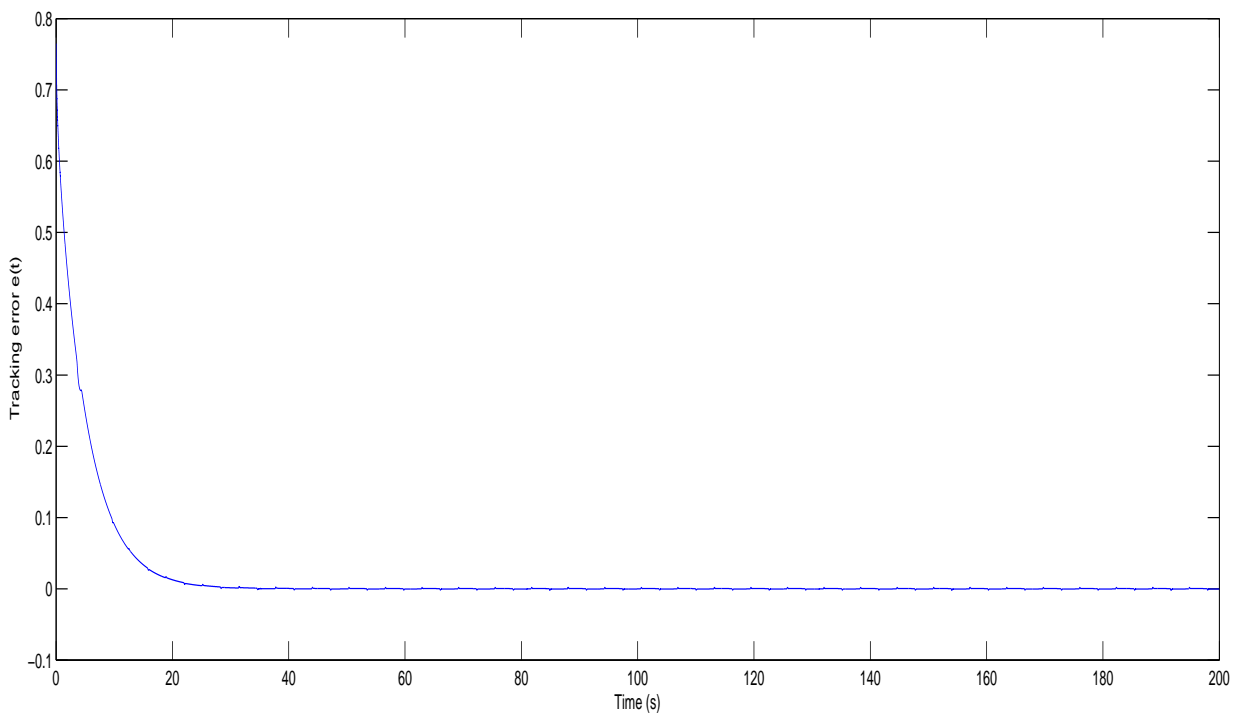

Figure 5.6: Tracking error $\left(\omega_{r}=2, y_{m}=\sin (t), \theta(0)=85 \% \theta^{*}, \Gamma=12\right)$

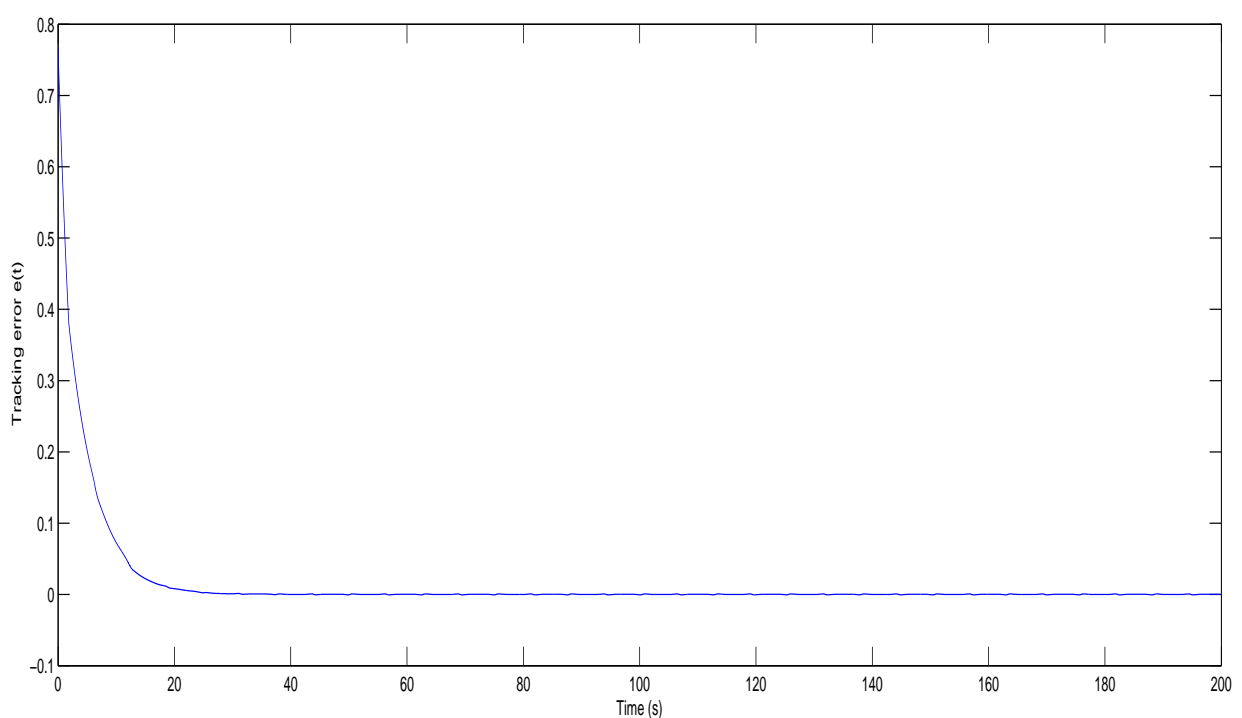

Figure 5.7: Tracking error $\left(\omega_{r}=2, y_{m}=\sin (0.5 t), \theta(0)=\theta^{*}, \Gamma=0\right)$ 


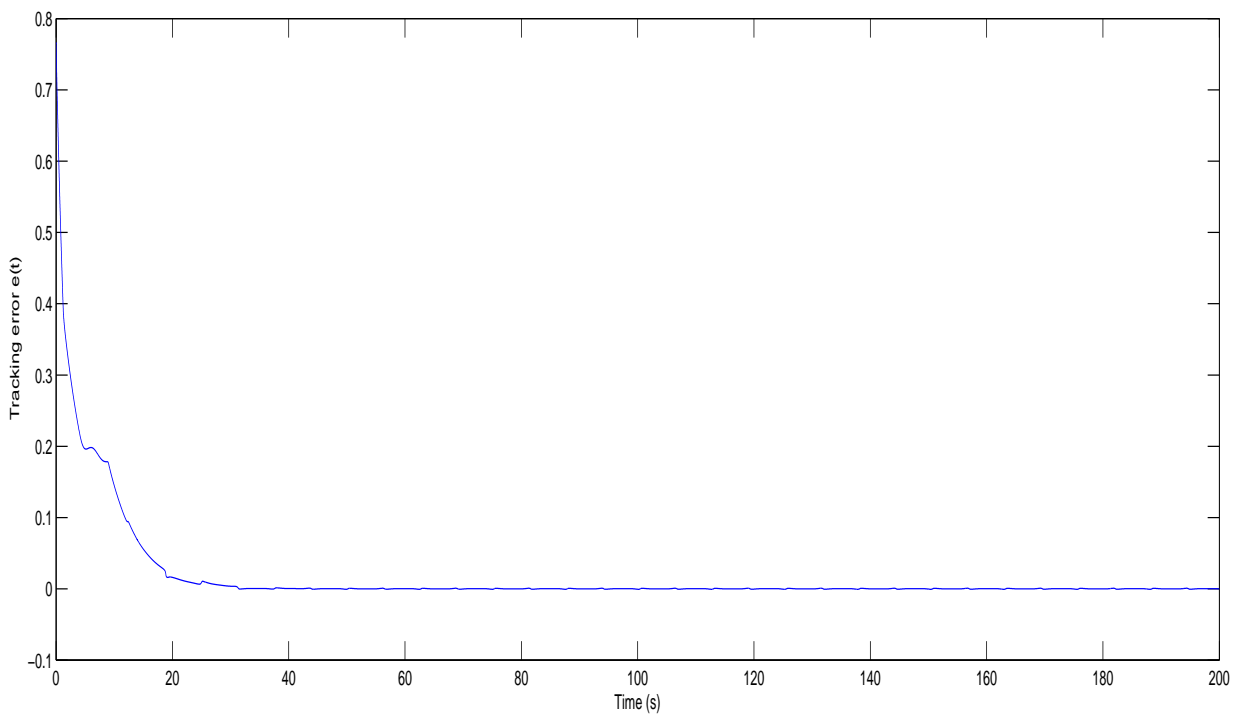

Figure 5.8: Tracking error $\left(\omega_{r}=2, y_{m}=\sin (0.5 t), \theta(0)=75 \% \theta^{*}, \Gamma=10\right)$

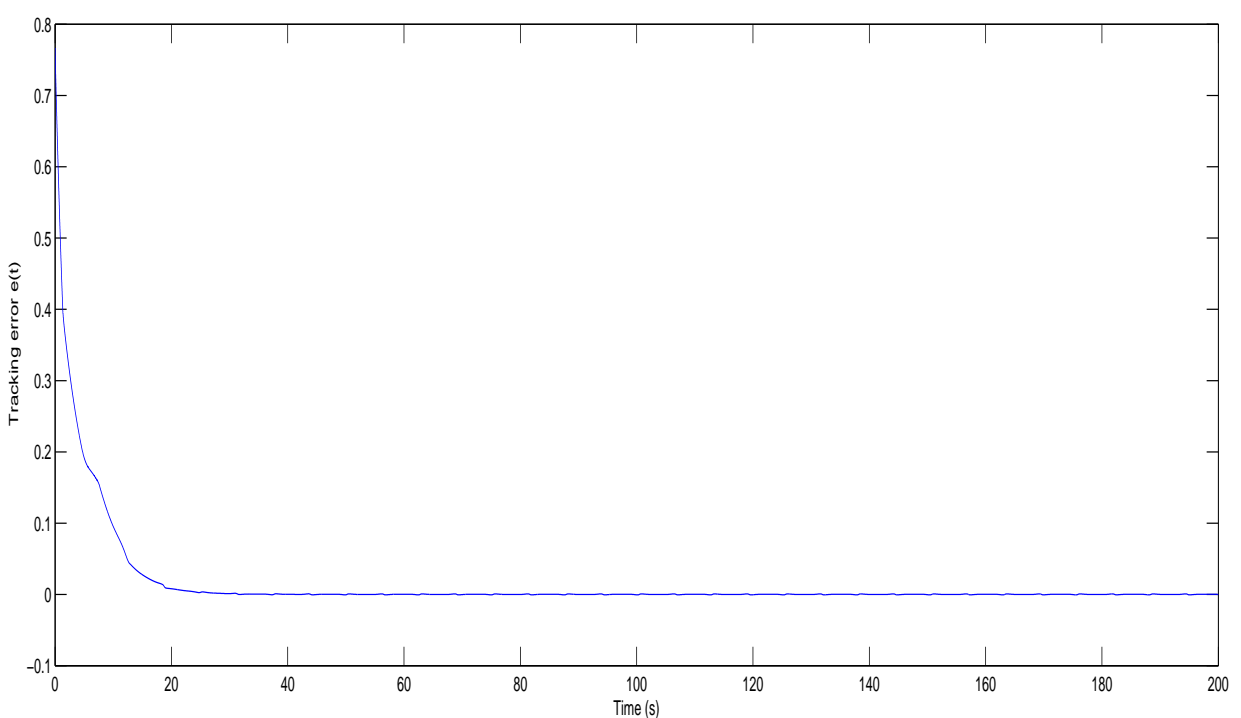

Figure 5.9: Tracking error $\left(\omega_{r}=2, y_{m}=\sin (0.5 t), \theta(0)=85 \% \theta^{*}, \Gamma=10\right)$ 
From the above simulation results, we can see that our system output can track the reference signals which are 1 and $\sin (t), \sin (0.5 t)$, the system tracking error will converge to zero in about 30 seconds. Both the nominal control design and adaptive control design can achieve our control objective, it shows that basic adaptive backstepping control design can cancel the effect of the system nonlinearity, so in next section, we will apply this method on our wind turbine system to see that we can still solve the problem of the system nonlinearity with time-varying parameters.

\subsection{Adaptive Backstepping Control for Wind Tur- bine System Model}

In this part, we continue presenting our adaptive backstepping control design with the wind turbine system model. The state space model has been introduced in Chapter 3, which can be written in the form:

$$
\begin{aligned}
& \dot{x}_{1}=x_{3} \\
& \dot{x}_{2}=x_{4} \\
& \dot{x}_{3}=\phi_{0}\left(x_{1}, x_{2}, x_{3}, x_{4}\right)+\theta_{10}^{*}(t) \phi_{1}\left(x_{1}, x_{2}, x_{3}, x_{4}\right)+b_{1} u \\
& \dot{x}_{4}=\phi_{2}\left(x_{1}, x_{2}, x_{3}, x_{4}\right)+\theta_{20}^{*}(t) \phi_{3}\left(x_{1}, x_{2}, x_{3}, x_{4}\right)+b_{2} u \\
& y=x_{2},
\end{aligned}
$$

where $\phi_{i}, i=0,1,2,3$ are some functions which are also differentiable with respect to $x_{1}, x_{2}, x_{3}, x_{4}$, the system state variables, $\theta_{11}^{*}, \theta_{22}^{*}$ are similar to the form of $\theta^{*}$ and $b_{1}, b_{2}$ are some unknown constant with known signs. The output of this wind turbine system is $x_{2}$, which refers to the pitch angle of the system. $x_{1}$ is the plunge displacement and $x_{3}, x_{4}$ are the derivatives of $x_{1}$ and $x_{2}$. In this wind turbine system, our control 
input $u$ can be considered as the 'elevator' angle deployed in the trailing edge of wind turbine blade, which can manipulate the pitch angle of this wind turbine system

Before we present our adaptive backstepping control design, we first do a simple analysis for this wind turbine system. We notice that in this wind turbine system, there are four system variables, and the output is $x_{2}$. So in our state space equation, we will pick out the main equation for our adaptive backstepping control design, which is given by

$$
\begin{aligned}
& \dot{x}_{2}=x_{4} \\
& \dot{x}_{4}=\phi_{2}\left(x_{1}, x_{2}, x_{3}, x_{4}\right)+\theta_{20}^{*}(t) \phi_{3}\left(x_{1}, x_{2}, x_{3}, x_{4}\right)+b_{2} u,
\end{aligned}
$$

Then we find out that there are still two equations, which are as follows:

$$
\begin{aligned}
& \dot{x}_{1}=x_{3} \\
& \dot{x}_{3}=\phi_{0}\left(x_{1}, x_{2}, x_{3}, x_{4}\right)+\theta_{10}^{*}(t) \phi_{1}\left(x_{1}, x_{2}, x_{3}, x_{4}\right)+b_{1} u,
\end{aligned}
$$

Piecewise zero dynamics In our adaptive backstepping control design, (5.19) can be considered as zero dynamics of this wind turbine system. From the state space model in Chapter 3, we generate several system parameters by choosing some certain number of $\sin \left(\omega_{r} t\right)$, when $\sin \left(\omega_{r} t\right)=-1,-0.5,0,0.5,1$, the zeros of the system are all stable. Therefore, we conclude that these two equations are stable when they are considered as the piecewise zero dynamics of this wind turbine system. We generate the system transfer functions with parameters of the nominal model with constant parameters. Therefore, with the chosen parameters $\sin \left(\omega_{r} t\right)=-1,-0.5,0,0.5,1$, we can generate the system zero equations which can be found in Table 5.1. From the Table 5.1, we can see that all the system zeros are in the open left-half of the 
complex plane, which means these equations can be considered as the piecewise zero dynamics. According to their boundedness, we can apply a similar adaptive control design in our wind turbine system.

Table 5.1: System Zero Polynomials

\begin{tabular}{|c|c|}
\hline \hline Value of $\sin \left(\omega_{r} t\right)$ & System Zero Polynomials \\
\hline-1 & $-0.8901 s^{2}-0.7957 s-84.7926$ \\
-0.5 & $-0.8901 s^{2}-0.8346 s-84.7926$ \\
0 & $-0.8901 s^{2}-1.0922 s-84.7926$ \\
0.5 & $-0.8901 s^{2}-0.8525 s-84.7926$ \\
1 & $-0.8901 s^{2}-0.8170 s-84.7926$ \\
\hline
\end{tabular}

Next, we will present the adaptive backstepping procedure for this wind turbine system model to achieve the desired system performance.

\subsubsection{Adaptive Backstepping Control Design}

Step 1: Introducing $z_{1}=x_{2}-y_{m}$ and $z_{2}=x_{4}-\beta_{1}$, where $\beta_{1}$ is also a design function to be determined, and the $z_{1}$ is written in the form:

$$
\dot{z}_{1}=z_{2}+\beta_{1}-\dot{y}_{m}
$$

And then, we will consider the first step Lyapunov function, which is given by

$$
V_{3}=\frac{1}{2} z_{1}^{2}
$$

Then the derivative of $(5.21)$ is

$$
\dot{V}_{3}=z_{1}\left(z_{2}+\beta_{1}-\dot{y}_{m}\right)
$$


Choosing the stabilizing function as follows:

$$
\beta_{1}=-c_{3} z_{1}+\dot{y}_{m}
$$

where $c_{3}>0$, from (5.20) and (5.22), we can get the equation

$$
\begin{aligned}
& \dot{z}_{1}=z_{2}-c_{3} z_{1} \\
& \dot{V}_{3}=-c_{3} z_{1}^{2}+z_{1}^{T} z_{2}
\end{aligned}
$$

Step 2: In this step, the time derivative of $z_{2}$ is given by

$$
\begin{aligned}
\dot{z}_{2} & =\dot{x}_{4}-\dot{\beta}_{1} \\
& =\phi_{2}\left(x_{1}, x_{2}, x_{3}, x_{4}\right)+\theta_{20}^{*}(t) \phi_{3}\left(x_{1}, x_{2}, x_{3}, x_{4}\right)+b_{2} u-\dot{\beta}_{1}
\end{aligned}
$$

Based on the state space model from Chapter 3, we can rewrite the form of (5.26), which is as follows:

$$
\dot{z}_{2}=\phi_{2}\left(x_{1}, x_{2}, x_{3}, x_{4}\right)+\sin \left(\omega_{r} t\right) \theta_{21}^{*} x_{2}+\sin \left(\omega_{r} t\right) \theta_{22}^{*} x_{3}+\sin \left(\omega_{r} t\right) \theta_{23}^{*} x_{4}+b_{2} u-\dot{\beta}_{1}
$$

where $\theta_{2 j}^{*}, j=1,2,3$ are some unknown constant parameters.

Then, (5.20) and (5.27) can be viewed to be stabilized by $\beta_{1}$ with respect to the Lyapunov function, which is given by

$$
\begin{aligned}
V_{4}= & V_{3}+\frac{1}{2} z_{2}^{2}+\frac{1}{2}\left(\theta_{21}-\theta_{21}^{*}\right) \Gamma_{1}^{-1}\left(\theta_{21}-\theta_{21}^{*}\right)+\frac{1}{2}\left(\theta_{22}-\theta_{22}^{*}\right) \Gamma_{2}^{-1}\left(\theta_{22}-\theta_{22}^{*}\right) \\
& +\frac{1}{2}\left(\theta_{23}-\theta_{23}^{*}\right) \Gamma_{3}^{-1}\left(\theta_{23}-\theta_{23}^{*}\right)+\frac{\left|b_{2}\right|}{2 \gamma_{1}} \tilde{\rho}_{1}^{2},
\end{aligned}
$$

where $\Gamma_{i}=\Gamma_{i}^{T}>0, i=1,2,3, \gamma_{1}$ is a positive constant, $\theta_{2 j}, j=1,2,3$ are the 
estimates of $\theta_{2 j}^{*}, j=1,2,3$, and $\tilde{\rho}_{1}=\hat{\rho}_{1}-\rho_{1}, \hat{\rho}_{1}$ is the estimate of $\rho_{1}=\frac{1}{b_{2}}$.

The time derivative of $V_{4}$ is

$$
\begin{aligned}
\dot{V}_{4}= & -c_{3} z_{1}^{2}+z_{1} z_{2}+z_{2} \dot{z}_{2}+\left(\theta_{21}-\theta_{21}^{*}\right) \Gamma_{1}^{-1}\left(\dot{\theta}_{21}-\dot{\theta}_{21}^{*}\right)+\left(\theta_{22}-\theta_{22}^{*}\right) \Gamma_{2}^{-1}\left(\dot{\theta}_{22}-\dot{\theta}_{22}^{*}\right) \\
& +\left(\theta_{23}-\theta_{23}^{*}\right) \Gamma_{3}^{-1}\left(\dot{\theta}_{23}-\dot{\theta}_{23}^{*}\right)+\frac{\left|b_{2}\right|}{\gamma_{1}} \tilde{\rho}_{1} \dot{\hat{\rho}}_{1} \\
= & -c_{3} z_{1}^{2}+z_{2}\left(x_{2}-y_{m}+\phi_{2}\left(x_{1}, x_{2}, x_{3}, x_{4}\right)+\sin \left(\omega_{r} t\right)\left(\theta_{21} x_{2}+\theta_{22} x_{3}+\theta_{23} x_{4}\right)+b_{2} u\right. \\
& \left.+c_{3}\left(\dot{x}_{2}-\dot{y}_{m}\right)-\ddot{y}_{m}\right)+\left(\theta_{21}-\theta_{21}^{*}\right) \Gamma_{1}^{-1}\left(\dot{\theta}_{21}-\Gamma_{1} z_{2} \sin \left(\omega_{r} t\right) x_{2}\right) \\
& +\left(\theta_{22}-\theta_{22}^{*}\right) \Gamma_{2}^{-1}\left(\dot{\theta}_{22}-\Gamma_{2} z_{2} \sin \left(\omega_{r} t\right) x_{3}\right)+\left(\theta_{23}-\theta_{23}^{*}\right) \Gamma_{3}^{-1}\left(\dot{\theta}_{23}-\Gamma_{3} z_{2} \sin \left(\omega_{r} t\right) x_{4}\right) \\
& +\frac{\left|b_{2}\right|}{\gamma} \tilde{\rho}_{1} \dot{\hat{\rho}}_{1}
\end{aligned}
$$

The adaptive laws for $\theta_{2 j}, j=1,2,3$ are given by

$$
\begin{aligned}
& \dot{\theta}_{21}=\Gamma_{1} z_{2} \sin \left(\omega_{r} t\right) x_{2} \\
& \dot{\theta}_{22}=\Gamma_{2} z_{2} \sin \left(\omega_{r} t\right) x_{3} \\
& \dot{\theta}_{23}=\Gamma_{3} z_{2} \sin \left(\omega_{r} t\right) x_{4}
\end{aligned}
$$

Then the time derivative of $V_{4}$ can be rewritten in the form:

$$
\begin{aligned}
\dot{V}_{4} & =-c_{3} z_{1}^{2}+z_{2}\left(b_{2} u-\chi_{1}(t)\right)+\frac{\left|b_{2}\right|}{\gamma_{1}} \tilde{\rho}_{1} \dot{\hat{\rho}}_{1} \\
& =-c_{3} z_{1}^{2}+z_{2} b_{2}\left(u-\rho_{1} \chi_{1}+\rho_{1} c_{4} z_{2}-\rho_{1} c_{4} z_{2}\right)+\frac{\left|b_{2}\right|}{\gamma_{1}} \tilde{\rho}_{1} \dot{\hat{\rho}}_{1} \\
& =-c_{3} z_{1}^{2}+z_{2} b_{2}\left(\hat{\rho}_{1}-\rho_{1}\right)\left(\chi_{1}(t)-c_{4} z_{2}\right)-c_{4} z_{2}^{2}+\frac{\left|b_{2}\right|}{\gamma_{1}} \tilde{\rho}_{1} \dot{\hat{\rho}}_{1}
\end{aligned}
$$

where $c_{4}>0$ and $\chi_{1}(t)=-\left(x_{2}-y_{m}+\phi_{2}\left(x_{1}, x_{2}, x_{3}, x_{4}\right)+\sin \left(\omega_{r} t\right)\left(\theta_{21} x_{2}+\theta_{22} x_{3}+\right.\right.$ $\left.\left.\theta_{23} x_{4}\right)+c_{3}\left(\dot{x}_{2}-\dot{y}_{m}\right)-\ddot{y}_{m}\right)$. 
Similarly, the controller for this wind turbine system is given by

$$
u(t)=\hat{\rho}_{1}\left(\chi_{1}(t)-c_{4} z_{2}\right)
$$

Then the adaptive laws for $\hat{\rho}_{1}(t)$ is as follows:

$$
\dot{\hat{\rho}}_{1}=-\gamma_{1} \operatorname{sign}\left(b_{2}\right) z_{2}\left(\chi_{1}(t)-c_{4} z_{2}\right)
$$

So the $\dot{V}_{4}$ eventually becomes

$$
\dot{V}_{4}=-c_{3} z_{1}^{2}-c_{4} z_{2}^{2}
$$

\section{Stability analysis}

Since $\dot{V}_{4} \leq 0$, we have that the error measure $V_{4}$ doesn't increase as a function of $t$, $z_{1}$ and $z_{2}$ and $\theta_{2 j}(t)-\theta_{2 j}^{*}$ are bounded, and so are $x_{2}$ and $\theta_{2 j}(t)$. Then, $\phi_{2}\left(x_{1}, x_{2}, x_{3}, x_{4}\right)$ is bounded, and so is $\beta_{1}$. From $z_{2}$, we see that $x_{4}$ is bounded. Since $x_{1}$ and $x_{3}$ are zero dynamics of the system, they are also bounded. It follows from (5.32) that $u(t)$ is bounded so that all signals in the closed-loop system are bounded.

We have the conclusions from (5.34) that

$$
c_{3} \int_{0}^{t} z_{1}^{2}(\tau) d \tau+c_{4} \int_{0}^{t} z_{2}^{2}(\tau) d \tau=V_{4}(0)-V_{4}(t)
$$

Since $V_{4}$ as a function of $t$ is bounded, it follows that $\int_{0}^{\infty} z_{1}^{2}(\tau) d \tau<\infty$ and $\int_{0}^{\infty} z_{2}^{2}(\tau) d \tau<$ $\infty$. From $\int_{0}^{\infty} z_{1}^{2}(\tau) d \tau<\infty$ and the boundedness of $\dot{z}_{1}(t)$, we can show that $\lim _{t \rightarrow \infty} z_{1}(t)=$ 0 , where $z_{1}(t)=y(t)-y_{m}(t)$. 
In summary, we have the following conclusion.

Theorem 5.2 The controller in (5.32) with adaptive laws (5.30) and (5.33) applied to the wind turbine system model guarantees that all closed-loop signals are bounded and the tracking error $e(t)=y(t)-y_{m}(t)$ goes to zero when $t$ goes to infinity.

\subsubsection{Simulation Study}

\section{Simulation conditions}

In this part, we present our simulation results with the parameters of our wind turbine system generated from Chapter 3. The physical meaning of the system output is pitch angle, therefore, the reference signal is chosen as same as what we do in the basic adaptive backstepping control design. In this simulation study, we chose $\phi_{0}\left(x_{1}, x_{2}, x_{3}, x_{4}\right)=-229.8782 x_{1}, \theta_{10}^{*}(t) \phi_{1}\left(x_{1}, x_{2}, x_{3}, x_{4}\right)=\left(3.4308-4.5096 \sin \left(\omega_{r} t\right)\right) x_{2}-$ $\left(2.6673+0.451 \sin \left(\omega_{r} t\right)\right) x_{3}-\left(0.00067+0.0067 \sin \left(\omega_{r} t\right)\right) x_{4}, \phi_{2}\left(x_{1}, x_{2}, x_{3}, x_{4}\right)=7.5865 x_{1}$, $\theta_{20}^{*}(t) \phi_{3}\left(x_{1}, x_{2}, x_{3}, x_{4}\right)=-\left(232.9684-0.911 \sin \left(\omega_{r} t\right)\right) x_{2}+\left(0.0891+0.0151 \sin \left(\omega_{r} t\right)\right) x_{3}-$ $\left(0.0171+0.0021 \sin \left(\omega_{r} t\right)\right) x_{4}$, and $b_{1}=15.7941, b_{2}=0.8901$. Also, in our simulation

results, we chose different numbers of $\omega_{r}$, which are $2,3,4$, to see that our adaptive control design can still achieve our control objective under different blade rotation cases. The simulation results of our wind turbine system are as follows. For the adaptive control case, when the reference signal $y_{m}=1$, our $\Gamma_{i}, i=1,2,3$, is 3 , when $y_{m}=\sin (t), \Gamma_{i}, i=1,2,3$, is 10 , when $y_{m}=\sin (0.5 t), \Gamma_{i}, i=1,2,3$, is 8 . When we present the results of our nominal control design, $\Gamma_{i}=0$. 


\section{Simulation results}

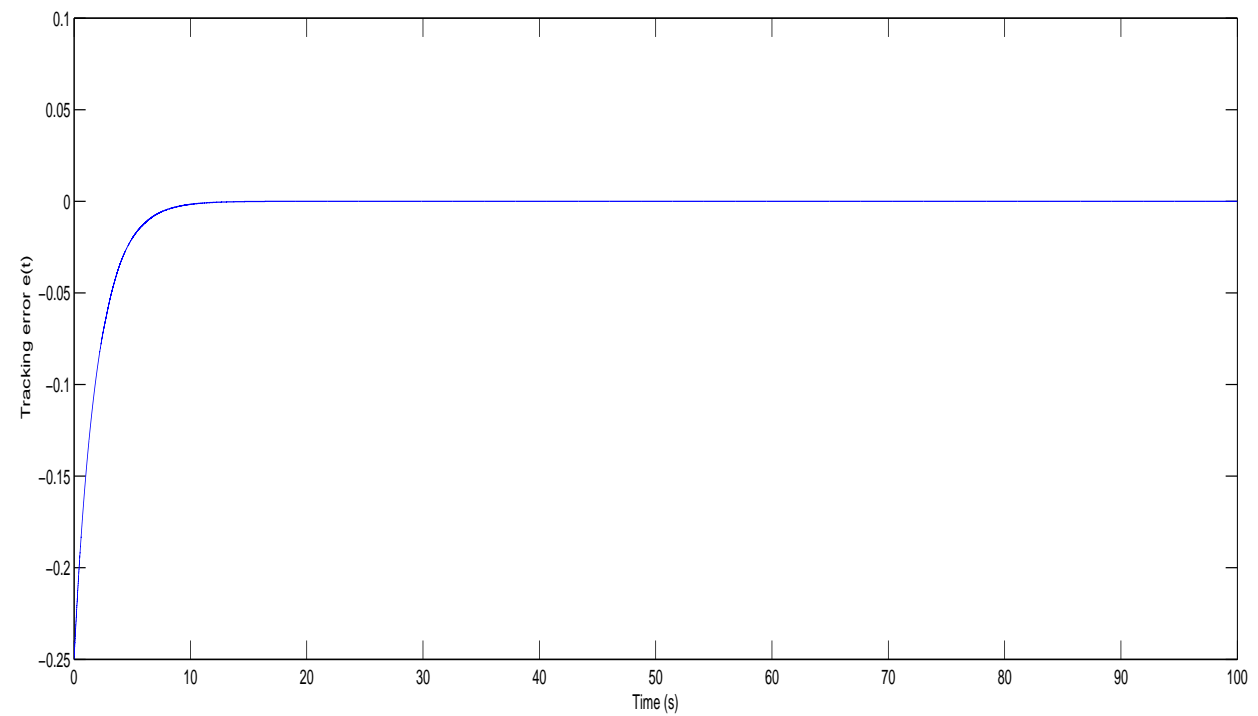

Figure 5.10: Tracking error (nominal control, $\omega_{r}=3, y_{m}=1$ )

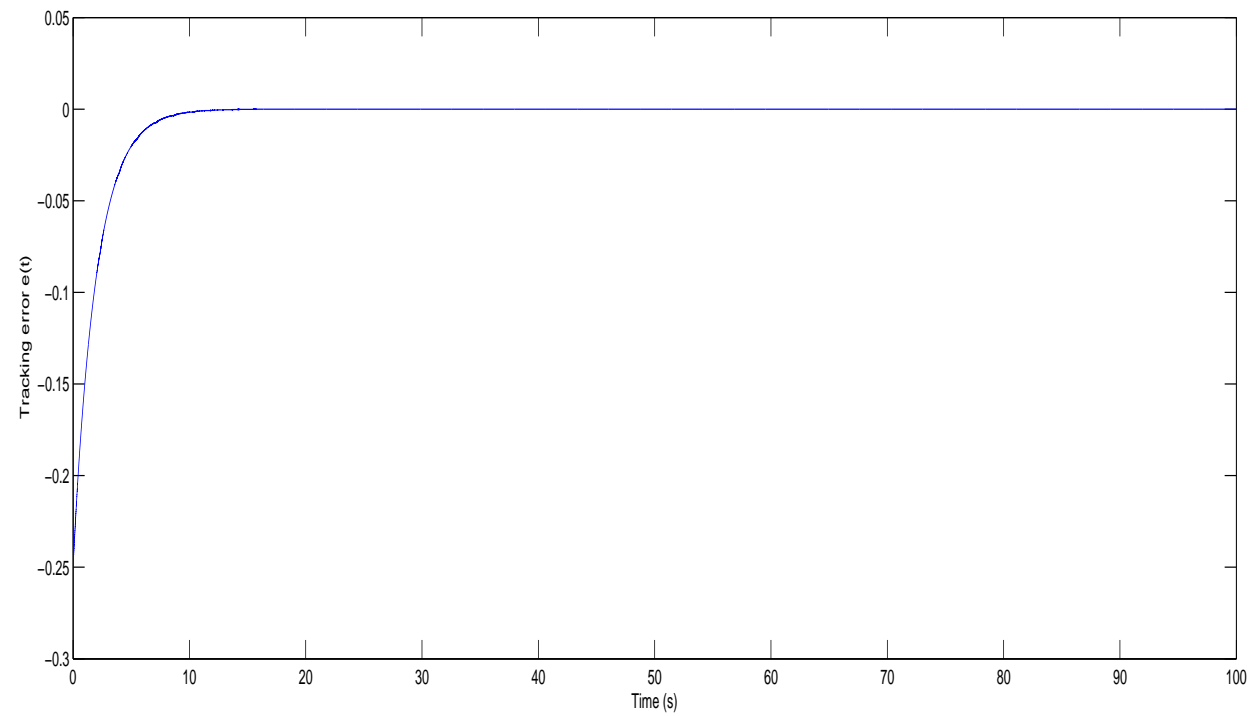

Figure 5.11: Tracking error (adaptive control, $\omega_{r}=3, y_{m}=1, \theta(0)=75 \% \theta^{*}$ ) 


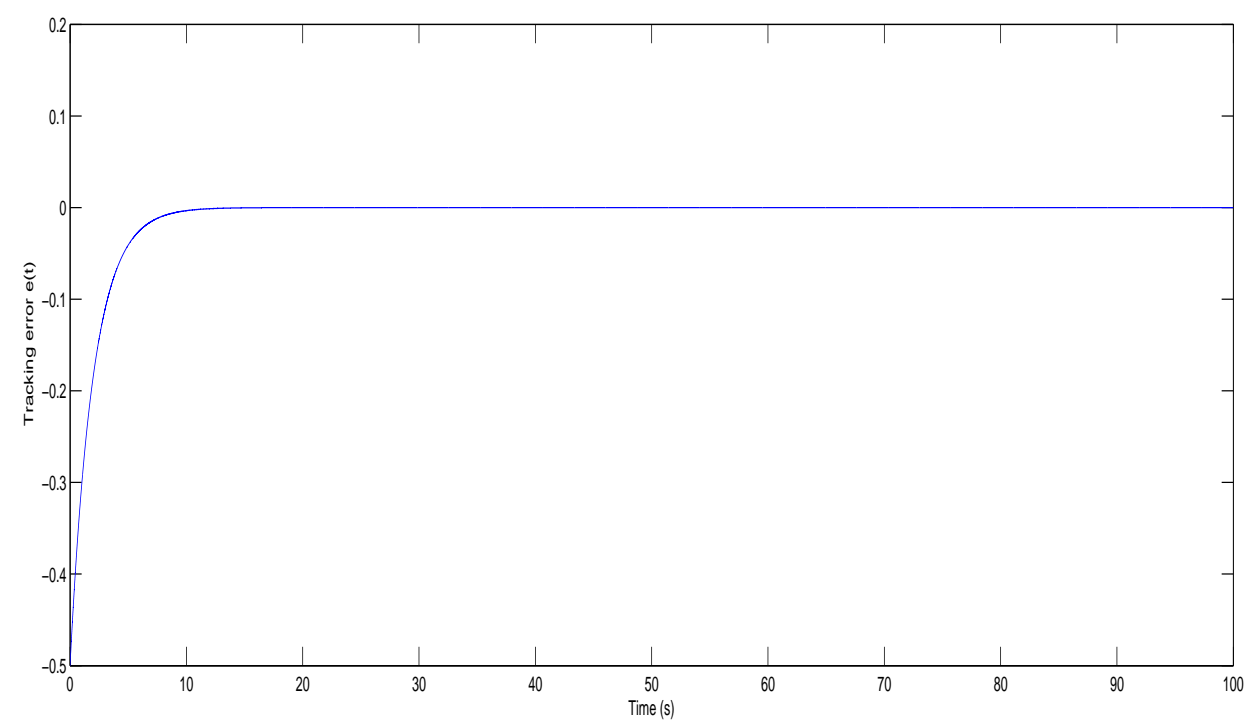

Figure 5.12: Tracking error $\left(\omega_{r}=4, y_{m}=1, \theta(0)=85 \% \theta^{*}\right)$

We can see that when the reference signal is chosen as 1 , which is a constant, the tracking error of the system will go zero in about 20 seconds. We also change the value of the blade rotation frequency, the tracking error still goes to zero in about 20 seconds, the simulation results show that when the reference signal is constant, our adaptive control design can get the desired system performance, which means the desired pitch angle will be obtained.

When the reference signals are chosen as the $\sin (t)$ and $\sin (0.5 t)$, there are some vibrations in simulation results, but as the time goes to $\infty$, the tracking error will go to zero eventually. 


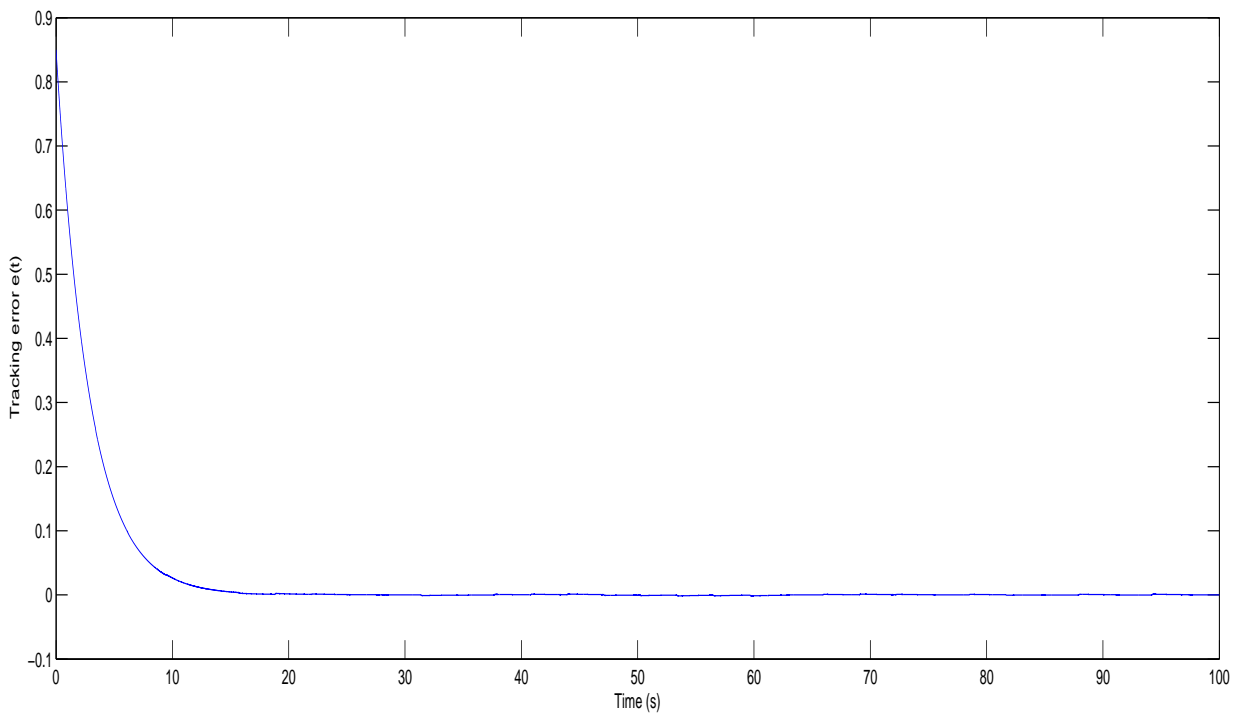

Figure 5.13: Tracking error (nominal control, $\omega_{r}=3, y_{m}=\sin (t)$ )

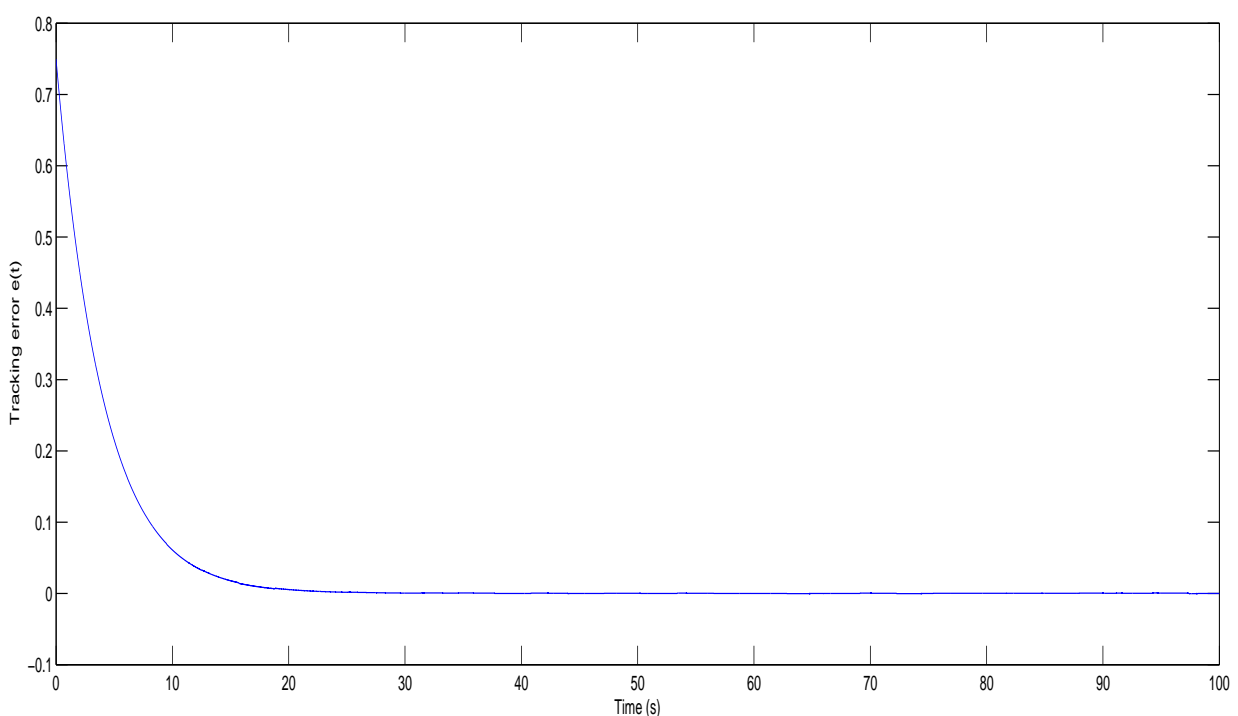

Figure 5.14: Tracking error (adaptive control, $\omega_{r}=3, y_{m}=\sin (t), \theta(0)=75 \% \theta^{*}$ ) 


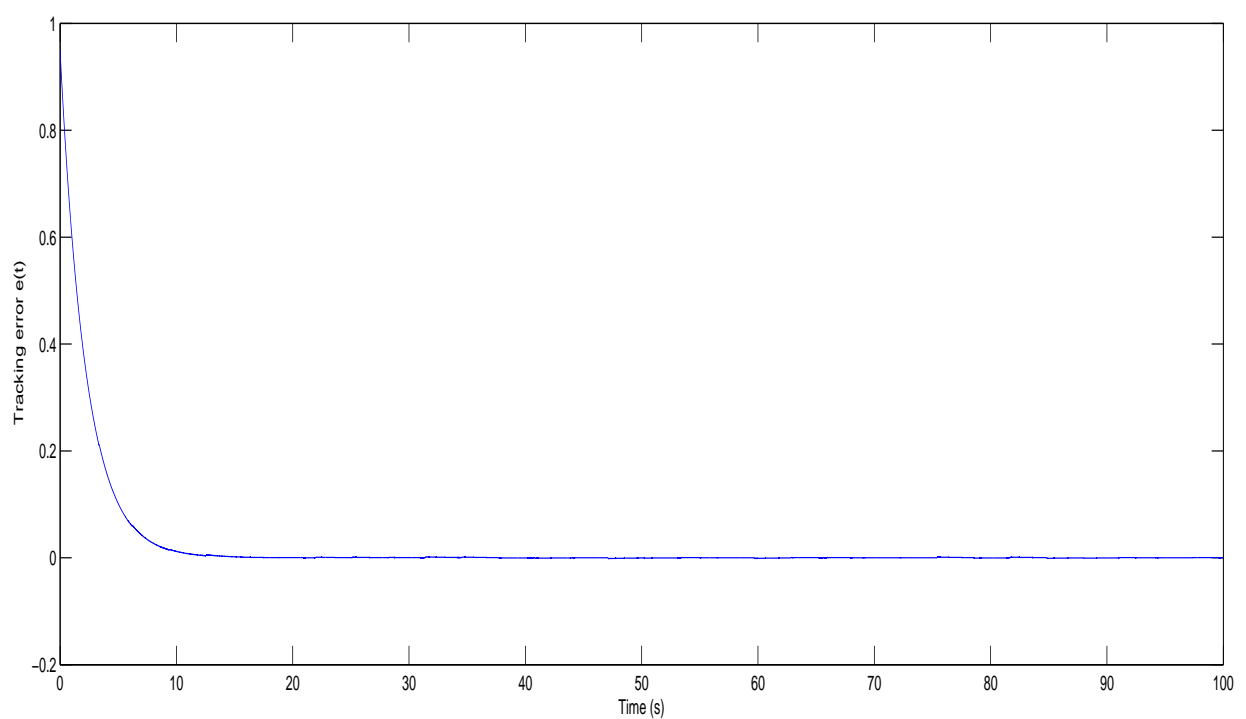

Figure 5.15: Tracking error $\left(\omega_{r}=4, y_{m}=\sin (t), \theta(0)=85 \% \theta^{*}\right)$

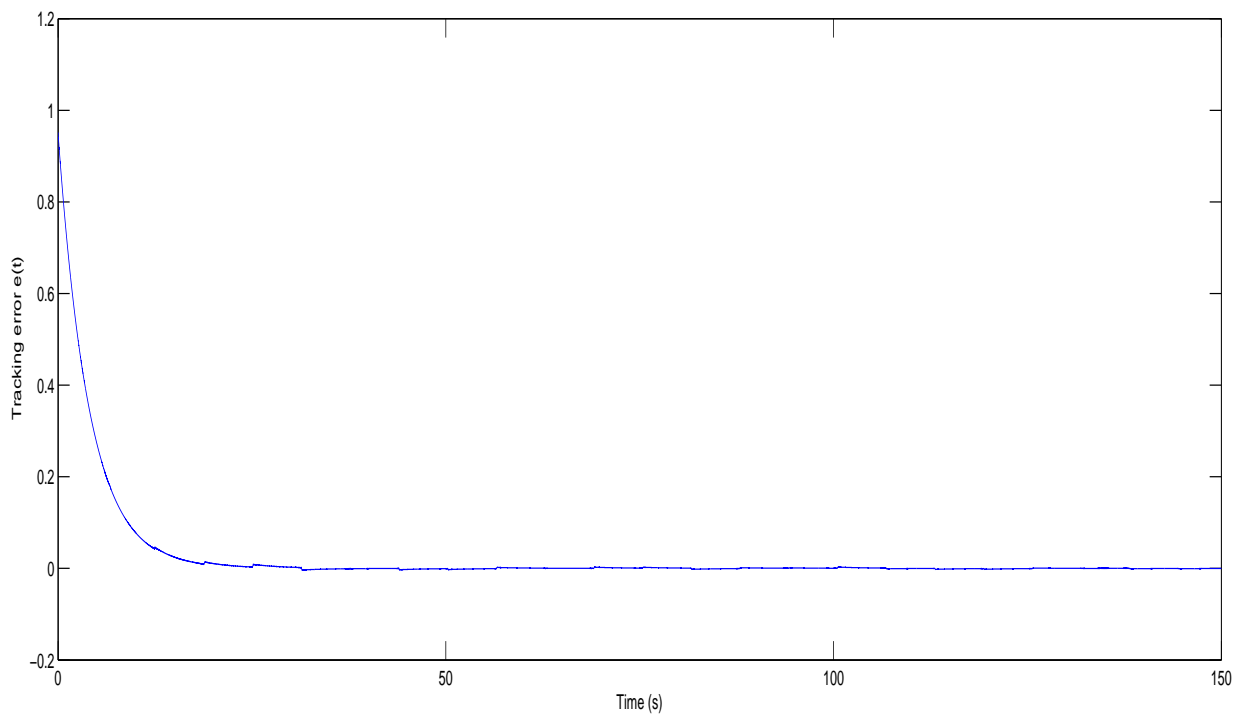

Figure 5.16: Tracking error (nominal control, $\omega_{r}=3, y_{m}=\sin (0.5 t)$ ) 


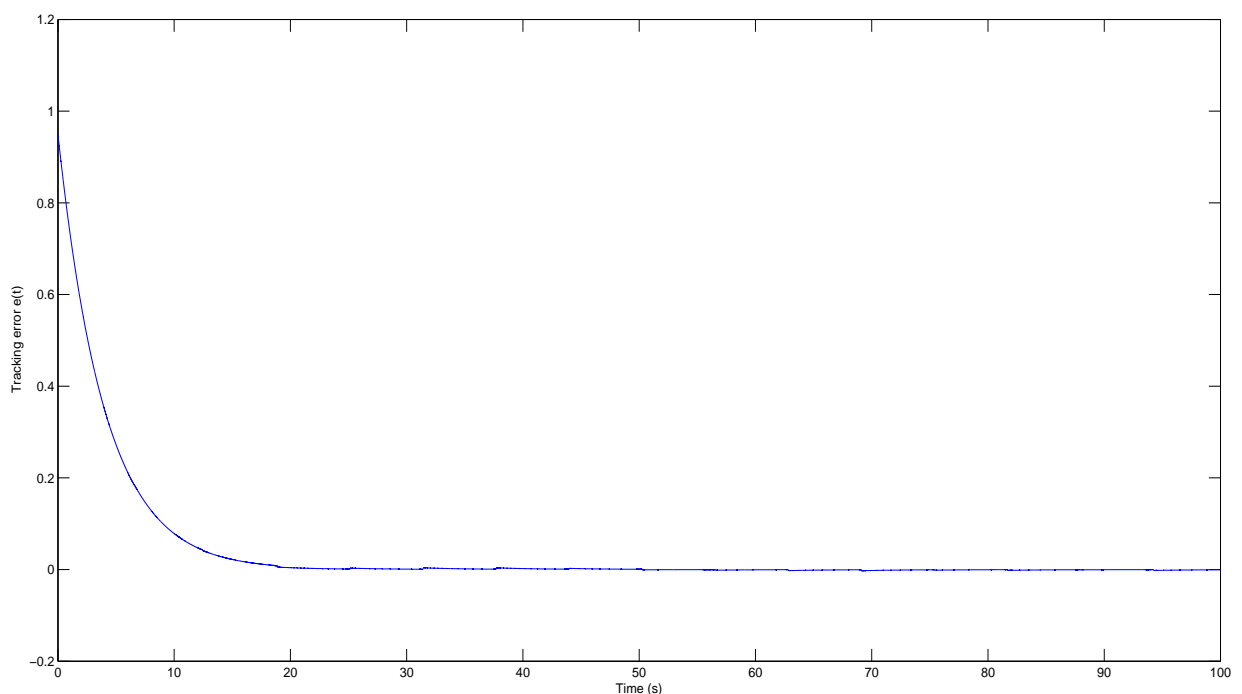

Figure 5.17: Tracking error (adaptive control, $\omega_{r}=3, y_{m}=\sin (0.5 t), \theta(0)=75 \% \theta^{*}$ )

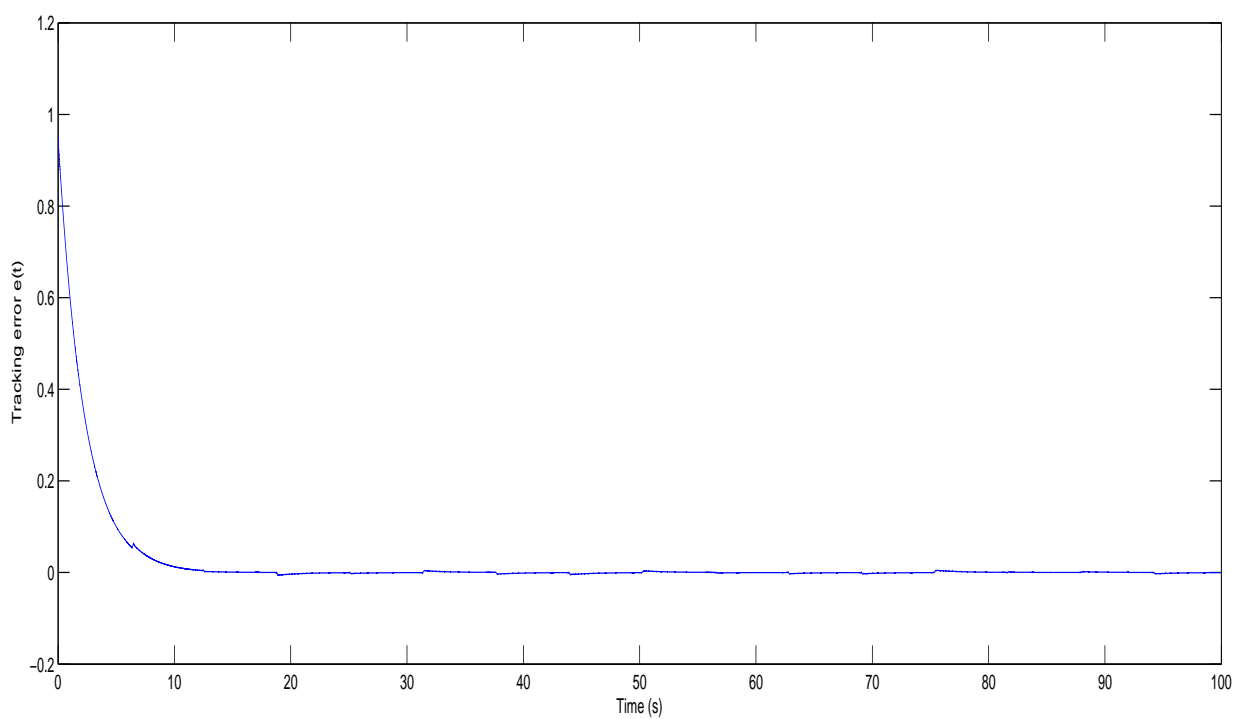

Figure 5.18: Tracking error $\left(\omega_{r}=4, y_{m}=\sin (0.5 t), \theta(0)=85 \% \theta^{*}\right)$ 
From our simulation results, we can see that our control design can successfully reach our control objective, which means the system output, pitch angle, can track the chosen reference signal. We can conclude that our adaptive backstepping control design can make the pitch angle of this wind turbine system track the reference signal to suppress the effect of blade rotation. Compared with the basic adaptive backstepping control design, simulation results of the wind turbine system are much more smooth. It takes less time to make our tracking error go to zero, which the desired system performance can be achieved.

\subsection{Comparison Study}

In this part, we first introduce a method of adaptive control design in [3], then we will make a comparison analysis between the way in [3] and our adaptive backstepping control design to see that why our adaptive control design is more advantageous and powerful.

\subsubsection{An Existing Adaptive Control Design}

System model. In [3], based on the state space equation of the NACA0012 airfoil model from Chapter 3, they present the system with input disturbance $u_{D}$, which is written in the form:

$$
\begin{aligned}
& \dot{x}=A(t) x+B u(t)+\Gamma(t) u_{D} \\
& y=C(t) x, y(0)=y_{0}
\end{aligned}
$$

where $y$ is the pitch angle, the system output, $A(t)$ is the state matrix, $B(t)$ is the control input matrix, $C(t)$ is the output matrix and $u_{D}$ is the disturbance input, 
which is from a disturbance generator. The disturbance input can be written in the form:

$$
\begin{aligned}
& u_{D}=\Theta z_{D} \\
& z_{D}=L \phi_{D}
\end{aligned}
$$

where $z_{D}$ is the disturbance state, $\phi_{D}$ is considered as a step input which is referred to a disturbance input, matrices $L$ and $\Theta$ both have appropriate dimensions. The authors assume that the disturbance input $\phi_{D}=1$, which is applied in this model.

Control design. Their control objective is to make the system output, $y$, converge to the desired pitch angle, $y^{*}$. Then, they demonstrated their controller form as follows:

$$
u=G_{e} y+G_{D} \phi_{D}
$$

where $G_{e}$ and $G_{D}$ are adaptive gain matrices with appropriate dimensions. The adaptive laws are given by

$$
\begin{gathered}
\dot{G}_{e}=-e_{y} e_{y}^{T} \gamma_{e} \\
\dot{G}_{D}=-e_{y} \phi_{D}^{T} \gamma_{D}
\end{gathered}
$$

where $e_{y}$ is the tracking error between system output and reference output, $\gamma_{e}, \gamma_{D}$ are arbitrary, positive definite matrices.

Design conditions. In their control design, they assume that there is a perturbation between system trajectory $(x, y, u)$ and the ideal system trajectory $\left(x^{*}, y^{*}, u^{*}\right)$ 
as $\Delta x=x-x^{*}, \Delta y=y-y^{*}, \Delta u=u-u^{*}$, the system can be written in the form:

$$
\begin{aligned}
& \Delta \dot{x}=A \Delta x+B \Delta u \\
& \Delta y=C \Delta x
\end{aligned}
$$

In order to reject the input disturbance, they assume that there is a matrix called $G_{D}^{*}$, it is satisfied that $B(t) G_{D}^{*}+\Gamma(t) \Theta L=0$. Then the equation in (5.38) is given

$$
\begin{aligned}
\Delta u & =\Delta G_{e} e_{y}+G_{e}^{*} e_{y}+\Delta G_{D} \phi_{D}=G_{e}^{*} y+w \\
w & =\Delta G \eta \\
\Delta & =\left[\Delta G_{e} \Delta G_{D}\right] \\
\eta & =\left[e_{y} \phi_{D}\right]
\end{aligned}
$$

So they substitute the equation (5.41) into (5.40), the new equations are as follows:

$$
\begin{aligned}
& \Delta \dot{x}=A_{c} \Delta x+B w \\
& \Delta y=C \Delta x=e_{y} \\
& A_{c}=A+B G_{e}^{*} C
\end{aligned}
$$

The authors use Kalman-Yacubovic(K-Y)Lemma to present their stability analysis with the chosen Lyapunov candidate function, which is like

$$
V(t, x)=x^{T} P(t) x
$$


where $P(t)$ is a symmetric positive definite matrix, which satisfies the condition

$$
\begin{aligned}
& \dot{P}(t)+A_{c}^{T}(t) P(t)+P(t) A_{c}(t)=-Q(t) \\
& P(t) B(t)=C^{T}(t)
\end{aligned}
$$

where $Q(t)$ is also a symmetric positive definite matrix, and $A_{c}(t)=A(t)+B(t) G_{e}^{*} C(t)$, $A(t), B(t), C(t)$ are all their system matrices. And they still need several assumptions:

1. There exist several positive constants $p_{\min }, p_{\max }, q_{\min }$

2. $p_{\min }\|x\|^{2} \leq V(t, x)=x^{T} P(t) x \leq p_{\max }\|x\|^{2}$ and $q_{\min } \leq x^{T} Q(t) x$.

Then they present the stability analysis results as follows:

$$
\begin{aligned}
\dot{V}(t, x) & =x^{T} P x+2 x^{T} P \dot{x} \\
& =x^{T}\left(\dot{P}+A_{c}^{T} P+P A_{c}\right) x+2 x^{T} P B w \\
& =x^{T} Q x+2<y, w> \\
V(\Delta G) & =\operatorname{tr}\left(\Delta \gamma^{-1} \Delta G^{T}\right) \\
\dot{V}(\Delta G) & =-2<y, w>
\end{aligned}
$$

Then they let $V(t, x, \Delta G)=V(t, x)+V(\Delta G)$, so they have the result like

$$
\begin{aligned}
\dot{V}(t, x, \Delta G) & =-x^{T} Q x+2<y, w>-2<y, w> \\
& =-x^{T} Q x \leq-q_{\text {min }}\|x\|^{2} \leq 0
\end{aligned}
$$




\subsubsection{Discussion}

Although our control design doesn't include the part of the disturbance, we will see that several assumptions are needed in order to achieve their control objective. In their control design procedure, we don't see any control design steps to deal with the time-varying parameters, it is not appropriate to chose the controller type in a linear form, in our adaptive backstepping control design, the controller type should be nonlinear, therefore, this nonlinear controller can cancel the negative effect brought by time-varying parts in the system.

In their adaptive stability theorem, several assumptions are made to ensure their boundedness of their signals, compared to our adaptive backstepping design, we don’t need such assumptions and we still can ensure the boundedness of input or our signals. Because when we face the problems which are referred to some certain applications, some strict conditions can't be satisfied and to deal with time-varying parts, [3] only says that their $(A(t), B(t), C(t))$ should satisfy K-Y lemma and be bounded, but adaptive backstepping control design can solve the problem when the system includes the time-varying part. It is very hard to say that some certain system matrices must satisfy K-Y lemma, then the adaptive control design is too limited to achieve our desired control objective. When they do the system stability analysis, their Lyapunov candidate function is $x^{T} P x$, there is no system error in their candidate function, which they mention in their control design procedure. So their system matrices can't satisfy the K-Y lemma condition, which means they can't prove that their control design can achieve their control objective. 


\subsection{Summary}

In this chapter, we demonstrate a new adaptive control design which is an extension of the adaptive backstepping control design. We achieve the control objective which the output pitch angle, $\theta$, can track the ideal reference signal. Since our system is in a nonlinear form, our designed controller is also nonlinear which can cancel effect of the system nonlinearity. We also make a comparison with the control design in [3], our control design is more powerful and advantageous which has less assumptions and limitations. 


\section{Chapter 6}

\section{Conclusions and Future Work}

\subsection{Summary and Conclusions}

\section{Summary}

In this thesis, we develop a new approach of control design, adaptive backstepping control design, to solve the problem of blade vibration of wind turbine systems. We present our simulation results in two cases. First, the blade rotation parameter is constant. Based on the existed adaptive output tracking control design, we see that our control design can generate the desired output, blade pitch angle. Second, the blade rotation parameter is time-varying. At the beginning, we still apply the same adaptive control design using in the first case. But we can't get the results which we want, the defined tracking error will become so far away and it is definitely harmful to the system. So we develop a new control design, which is the adaptive backstepping control design. There are two steps in our design procedure, which we notice that the controller is also nonlinear. In our simulation study, the defined tracking error will go to zero finally with the satisfied reference signal, which are constant, $\sin (t)$ and $\sin (0.5 t)$. 
In our control design, the disturbance is neglected in order to get the control objective. It is disappointing that the disturbance, such as gust or other environmental influences, is also a serious factor which can harm the efficiency of the wind turbine power generation. It needs to be done for the wind turbine design with disturbance rejection. In [3], the assumed disturbance model is from a disturbance generator which can be used in the further study.

\section{Conclusions}

In this thesis, there are several conclusions we can obtain as follows:

1. The blade vibration problem can be a new version in studying the control problem of wind turbine systems. It is crucial that we can improve the efficiency of the power generation by realizing the vibration suppression.

2. The model reference adaptive control designs can't solve the problem that there are time-varying parameters in our desired systems. But we can see that if our system matrices are constant, the model reference adaptive control can obtained the desired system performance with the appropriate gains successfully.

3. Our adaptive backstepping control design can cancel the effect of the nonlinearity of wind turbine system with time-varying parameters, before applying this adaptive control method, we should know the form of the nonlinear functions in the systems, such as the function of $\sin \left(\omega_{r} t\right)$.

4. There are several factors which can be regarded as the disturbances in the wind turbine systems. Besides the gusts or mechanical problem, the hystereses in smart actuators also should be concerned. We can apply the adaptive inverse control design to solve such a problem. 


\subsection{Future Work}

In our future work, first, we need to fix the accuracy of the wind turbine models. It is impossible to generate the control design without the disturbance. We need do a lot of work on the systems with disturbances, which is much more closer to the real system. Therefore, we need to develop new adaptive control design with the disturbance rejection. Then, we can focus on the control design on the hysteresis of the smart materials. Although, in our thesis, the adaptive inverse control design against hysteresis characteristics is not included in our control design, it is need to be done in our future work. Adaptive inverse control design can make the smart actuators more efficient and this control design can solve the problem of the actuator imperfections. Furthermore, there are a lot of control problems in wind turbine systems, besides the control issue of the blade pitch angle, like the blade attack angle or the turbine yaw

adaptive control design. In the future work, there are many potential topics in wind turbine systems which we can focus on. 


\section{Bibliography}

[1] Thomas W. Strganac,Jeonghwan Ko,David E. Thompson, "Identification and control of limit cycle oscillations in aeroelastic systems," Journal of Guidance, Control, and Dynamics vol. 23, no. 6, Nov-Dec 2000.

[2] K.Johnson, L.Fingersh, M.Balas, and L.Pao, "Methods for increasing region 2 power capture on a variable speed wind turbine," J.Solar Energy Eng, vol.126 no.4, pp.1092-1100, 2004.

[3] Nailu Li, Mark J.Balas, "Adaptive flow control of wind turbine blade using Microtabs with unsteady aerodynamic loads," IEEE Green Technologies Conference, 2013.

[4] G. Tao, Adaptive Control Design and Analysis, John Wiley and Sons Inc., Hoboken, NJ, 2003.

[5] Daonchun Li, Jinwu Xiang, Shijun Guo, "Adaptive control of a nonlinear aeroelastic system," Elsevier Masson SAS, Aerospace Science and Technology, 15th, 2011.

[6] E.H. Dowell, J.Edwards, T.Strganac, "Nonlinear aeroelasticity," Journal of Aircraft 40th, 2003.

[7] B.H.K. Lee, S.J. Price, Y.S Wong, "Nonlinear aeroelastic analysis of airfoil: Bifurcation and chaos," Progress in Aerospace Science 35th, 1999. 
[8] L. Djayapertapa, C.B. Allen, "Numerical simulation of transonic flutter suppression by active control," 20th Applied Aerodynamics Conference, AIAA, St Louis, 2002.

[9] C.B. Allen, C.L. Fenwick, N.V. Taylor, L. Djayapertapa, "Investigation of flutter suppression by active control," 21st AIAA Applied Aerodynamics Conference,, June 2003.

[10] Leithead, W., and Connor, B., "Control of Variable Speed Wind Turbines: Dynamic Models," Int. J. Control, pp. 1173-C1188, 2000.

[11] Fadaeinedjad, R., Moschopoulos, G., and Moallem, M., "Investigation of Voltage Sag Impact on Wind Turbine Tower Vibrations," Wind Energy, pp. 351-C375, 2008.

[12] J. F. Manwell, J. G. McGowan, A. L. Rogers, Wind energy explained Theory, Design and Application, John Wiley and Sons Inc., Hoboken, NJ, 2009.

[13] S. Lindenberg, B. Smith, K. O’Dell, and E. DeMeo, "20 Percent Wind Energy by 2030: Increasing Wind Energy's Contribution to U.S. Electricity Supply," U.S Department of Energy, July, 2008.

[14] R. Redlinger, P. Andersen, and P. Morthorst, "Wind energy in the 21st century: Economics, policy, technology, and the changing electricity industry," Palgrave Macmillan, 2002.

[15] Robert Gasch, Jochen Twele, Wind power plants. Fundamentals, design, construction and operation, Springer, New York, 2012.

[16] Eggleston, D. M. and Stoddard, F. S., Wind Turbine Engineering Design, Van Nostrand Reinhold, New York, 1987. 
[17] Johnson, G. L. , Wind Energy Systems, Prentice Hall, Englewood Cliffs, NJ, 1985.

[18] Mohit Singh, Surya Santoso, "Dynamic models for Wind Turbines and Wind Power Plants," National Renewable Energy Laboratory, January 11, 2008 - May 31, 2011.

[19] Emrah Kulunk, Aerodynamics of Wind Turbines,Fundamental and Advanced Topics in Wind Power, ISBN: 978-953-307-508-2, InTech, 2011.

[20] A. Betz, "Wind Energy and its Exploitation by Windmills," Gottingen: Vandenhoeck und Ruprccht, p. 64, 1926.

[21] H. Glauert, "Windmills and fans," Aerodynamic theory, vol. 3, 1995.

[22] B. Kuo, Automatic control systems, Prentice Hall, 1995.

[23] V. Akhmatov, Induction generators for wind power, Paul, Co. Pub, Consortium, 2007.

[24] Janaka B. Ekanayake, Lee Holdsworth, Xueguang Wu and Nicholas Jenkins, "Dynamic Modeling of Doubly Fed Induction Generator Wind Turbines," IEEE Transactions on Power Systems, vol.18, No.2, pp. 803-809, May. 2003.

[25] Istvan Erlich, Jrg Kretschmann, Jens Fortmann, Stephan Mueller-Engelhardt and Holger Wrede, "Modeling of Wind Turbine Based on Doubly-Fed Induction Generators for Power System Stability Studies," IEEE Transactions on Power Systems, vol.22, No.3, pp. 909-919, August 2007.

[26] Arantxa Tapia, Gerardo Tapia, J. Xabier Ostolaza and Jos Ramn Senz, "Modeling and Control of a Wind Turbine Driven Doubly Fed Induction Generator," IEEE Transactions on Energy Conversion, vol. 18, No. 2, pp. 194-204, June. 2003. 
[27] Hua Shan, Li Jiang, Chaoqun Liu, Michael Love and Brant Maines, "Numerical Study of Passive and Active Flow Separation Control over a NACA0012 Airfoil," Technical Report, University of Texas at Arlington, 2007.

[28] Ram Venkataraman Iyer, Xiaobo Tan, and P. S. Krishnaprasad, "Approximate Inversion of the Preisach Hysteresis Operator With Application to Control of Smart Actuators," IEEE Transactions On Automatic Control, vol. 50, No. 6, June. 2005.

[29] Xiaobo Tan and John S. Baras, "Adaptive Identification and Control of Hysteresis in Smart Materials," IEEE Transactions On Automatic Control, vol. 50, No. 6, June 2005.

[30] Scott J. Johnson, Jonathon P. Baker, C. P. van Dam and Dale Berg, "An overview of active load control techniques for wind turbines with an emphasis on microtabs," Research Article, Wind Energy, pp. 239-253, August. 2009.

[31] Y.C. Li, K.-S. Choi, J.J. Wang, "Gurney Flap: Lift enhancement, mechanisms and applications," ScienceDirect, Progress in Aerospace Sciences, Elsevier Ltd., November. 2007.

[32] Farzad Mohebbi and Mathieu Sellier, "On the Kutta Condition in Potential Flow over Airfoil," Journal of Aerodynamics, Hindawi Publishing Corporation, 2014.

[33] Yen, D.T, van Dam,C.P, Bruchle, F, Smith,R.L, and Collins, S.D, "Active Load Control and Lift Enhancement Using MEM Translational Tabs," Fluids 2000, AIAA-2000-2422, Denver, CO, June. 2000.

[34] Yen-Nakafuji, D.T, van Dam, C.P, Smith, R.L, and Collins, S.D, "Active Load Control for Airfoils Using Microtabs," Journal of Solar Energy Engineering, vol. 123, No. 4, pp. 282-289, Nov. 2001. 
[35] Standish, K.J, and van Dam, C.P, "Computational Analysis of a Microtab-Based Aerodynamic Load Control System for Rotor Blades," J. American Helicopter Society, vol. 50, No. 3, pp. 249-258, Jul. 2005.

[36] S.O.R. Moheimani and G. C. Goodwin, "Guest editorial introduction to the special issue on dynamics and control of smart structure," IEEE Trans. Control Syst.Technol., vol.9, pp.3-4, 2001.

[37] L.0.Chua and S.C.Bass, "A generalized hysteresis model," IEEE Trans.Circuit Theory, vol.CT-19, pp.36-48, Jan. 1972.

[38] M.A.Krasnosel and A.V.Pokrovskii, Systems with Hysteresis, Berlin:SpringerVerlag, 1983.

[39] N.D.Vaughan and J.B.Gamble, "The modelling and simulation of a proportional solenoid valve," Presented at the Winter Ann. Meet. Amer. Soc. Mech. Eng., Dallas,TX, Nov. 1990.

[40] Gianluca Diodati, Monica Ciminello and Antonio Concilio, "Piezoelectric and electromagnetic solutions aimed at realizing an active Gurney flap," Journal of Intelligent Material Systems and Structures, Sage, August. 2012.

[41] Gang Tao and Petar V.KokotoviC, "Adaptive Control of Plants with Unknown Hysterese," IEEE Transactions On Automatic Control, vol.40, No.2, February. 1995.

[42] Gang Tao and P.V.Kokotovic, "Adaptive control of plants with unknown deadzones," 1992 Amer. Contr. Conf, pp.2710-2714, Chicago, IL, June. 1992.

[43] Gang Tao and P.V.Kokotovic, "Adaptive control of systems with backlash," $A u$ tomatica, vol.29. pp.323-335, Mar. 1993. 
[44] Norman S. Nise, Control Systems Engineering, 6th Edition, John Wiley and Sons Inc., Hoboken, NJ, 2011.

[45] HAkan Fortell, "Calculation of Zero Dynamics using the Ritt Algorithm," Proceedings of the 33rd IEEE Conference on Decision and Control, December. 1994.

[46] C.I. Byrnes, I.G. Lauko, D.S. Gilliam, and V.I. Shubov, "Zero Dynamics for Relative Degree One SISO Distributed Parameter Systems," Proceedings of the 37th IEEE Conference on Decision and Control, December. 1998.

[47] C.S. Jang, J.C. Ross, and R.M. Cummings, "Computational Evaluation of an Airfoil with a Gurney Flap," AIAA 10th Applied Aerodynamics Conference, Palo Alto CA, AIAA Paper 92-2708, Jun. 1992.

[48] James Hamilton and Drew Liming, "Careers in Wind Energy," U.S. Bureau of Labor Statistics: Wind Energy, Sep. 2010. 